\title{
WestVirginiaUniversity
}

THE RESEARCH REPOSITORY @ WVU

Graduate Theses, Dissertations, and Problem Reports

2013

\section{Aerodynamics Investigation of Faceted Airfoils at Low Reynolds Number}

Zachary G. Napolillo

West Virginia University

Follow this and additional works at: https://researchrepository.wvu.edu/etd

\section{Recommended Citation}

Napolillo, Zachary G., "Aerodynamics Investigation of Faceted Airfoils at Low Reynolds Number" (2013). Graduate Theses, Dissertations, and Problem Reports. 4985.

https://researchrepository.wvu.edu/etd/4985

This Thesis is protected by copyright and/or related rights. It has been brought to you by the The Research Repository @ WVU with permission from the rights-holder(s). You are free to use this Thesis in any way that is permitted by the copyright and related rights legislation that applies to your use. For other uses you must obtain permission from the rights-holder(s) directly, unless additional rights are indicated by a Creative Commons license in the record and/ or on the work itself. This Thesis has been accepted for inclusion in WVU Graduate Theses, Dissertations, and Problem Reports collection by an authorized administrator of The Research Repository @ WVU. For more information, please contact researchrepository@mail.wvu.edu. 


\title{
Aerodynamics Investigation of Faceted Airfoils at Low Reynolds Number
}

\author{
Zachary G. Napolillo \\ Thesis submitted to the \\ College of Engineering and Mineral Resources \\ at West Virginia University \\ in partial fulfillment of the requirements for the degree of \\ Masters of Science \\ In \\ Aerospace Engineering
}

Wade W. Huebsch, Ph.D., Chair

John M. Kuhlman, Ph.D.

Patrick Browning, Ph.D.

Department of Mechanical and Aerospace Engineering

Morgantown, West Virginia

2013

Keywords: Micro Air Vehicle, Unmanned Aerial Vehicle, Low Reynolds Number, Faceted Airfoils, ShopFoil, Tip Vortex, Effective Angle of Attack, Wind Tunnel, Flow Visualization, Computational Fluid Dynamics 


\section{Abstract \\ Aerodynamics Investigation of Faceted Airfoils at Low Reynolds Number}

Zachary G. Napolillo

The desire and demand to fly farther and faster has progressively integrated the concept of optimization with airfoil design, resulting in increasingly complex numerical tools pursuing efficiency often at diminishing returns; while the costs and difficulty associated with fabrication increases with design complexity. Such efficiencies may often be necessary due to the power density limitations of certain aircraft such as small unmanned aerial vehicles (UAVs) and micro air vehicles (MAVs).

This research, however, focuses on reducing the complexity of airfoils for applications where aerodynamic performance is less important than the efficiency of manufacturing; in this case a Hybrid Projectile. By employing faceted sections to approximate traditional contoured wing sections it may be possible to expedite manufacturing and reduce costs.

We applied this method to the development of a low Reynolds number, disposable Hybrid Projectile requiring a 4.5:1 glide ratio, resulting in a series of airfoils which are geometric approximations to highly contoured cross-sections called ShopFoils. This series of airfoils both numerically and experimentally perform within a 10\% margin of the SD6060 airfoil at low Re.

Additionally, flow visualization has been conducted to qualitatively determine what mechanisms, if any, are responsible for the similarity in performance between the faceted ShopFoil sections and the SD6060. The data obtained by these experiments did not conclusively reveal how the faceted surfaces may influence low Re flow but did indicate that the ShopFoils did not maintain flow attachment at higher angles of attack than the SD6060. Two reasons are provided for the unexpected performance of the ShopFoil: one is related to downwash effects, which are suspected of placing the outer portion of the span at an effective angle of attack where the ShopFoils outperform the SD6060; the other is the influence of the tip vortex on separation near the wing tips, which possibly provides a 'comparative advantage' to the ShopFoil because it has more to gain from a reduction in its pressure drag component. 


\section{Acknowledgements}

I would like to thank my committee members: Dr. Wade W. Huebsch, Dr. John M. Kuhlman, and Dr. Patrick H. Browning for allowing me the opportunity to participate in the graduate research program at WVU and for their guidance in developing my understanding of aerodynamics and experimentation.

Additionally, I appreciative of Dr. Loth for cultivating my conceptual understanding of fluid dynamics and thermodynamics as well as providing academic advice. Further, my thanks to Shanti Hamburg and Chris Menchini for offering guidance in developing my skills and understanding of Meshing and CFD. I appreciate the assistance from Dr. Xingbo Liu and Dr. Koorosh Mirfakhraie for recommending me to the WVU graduate engineering program. Thanks to Brian Parker for helping with my wind tunnel test preparations. To all of the influences throughout my life which gave rise to my interest in aeronautics, spaceflight, and the sciences. 


\section{Table of Contents}

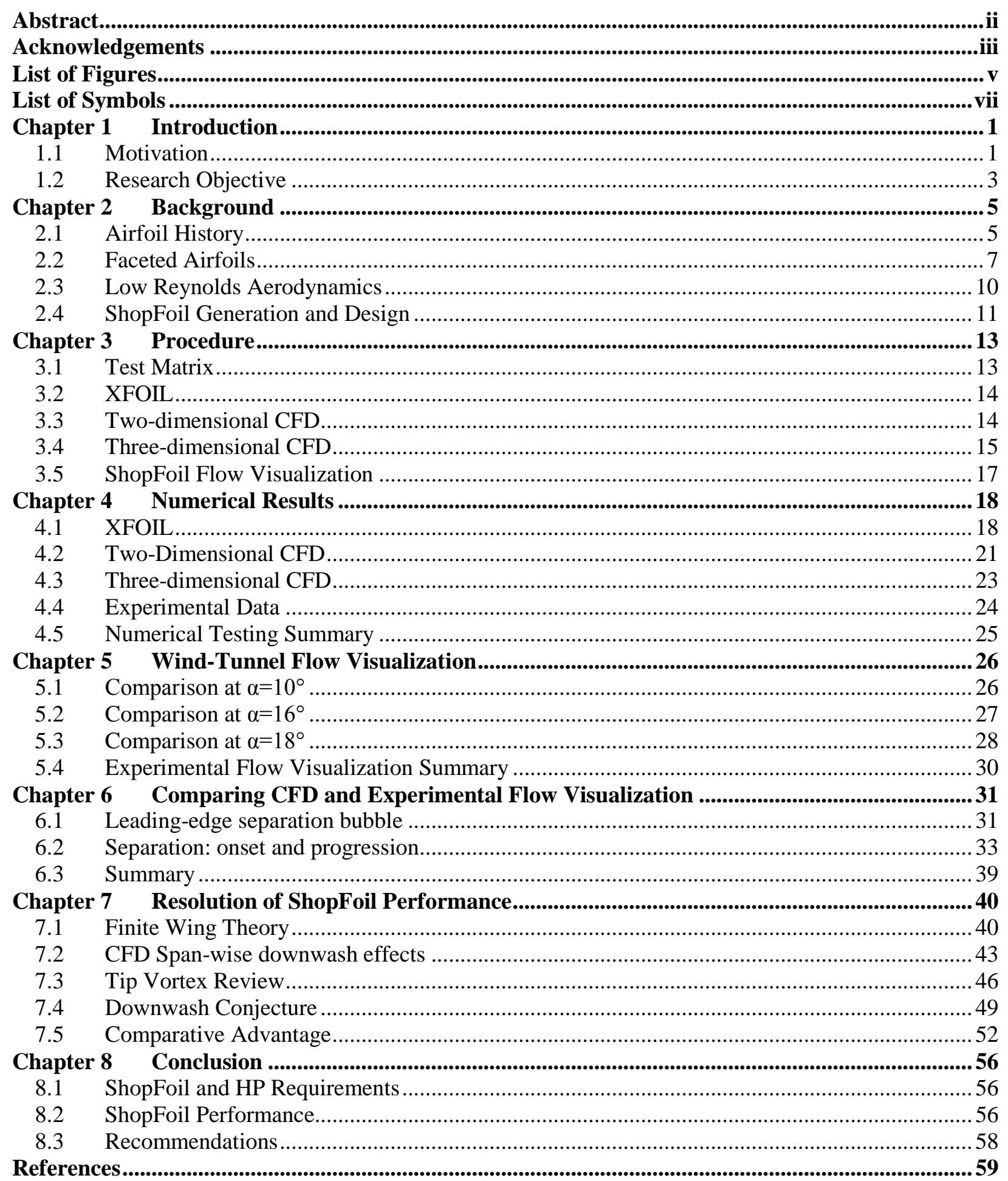




\section{List of Figures}

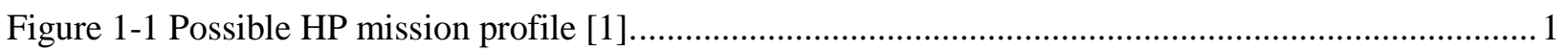

Figure 2-1 Phillips's first cambered airfoil, patented in 1891 (Edited)..................................................5

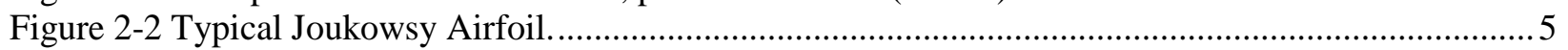

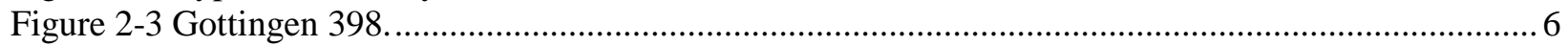

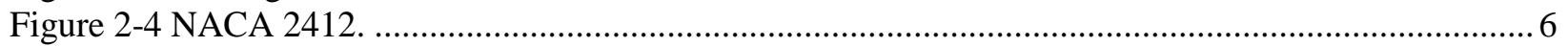

Figure 2-5 Faceted upper surface and trailing edge of a return boomerang. …....................................... 7

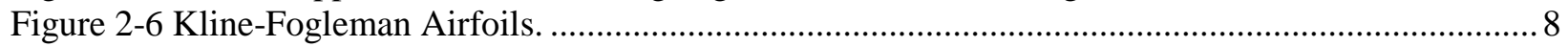

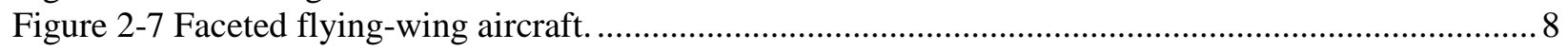

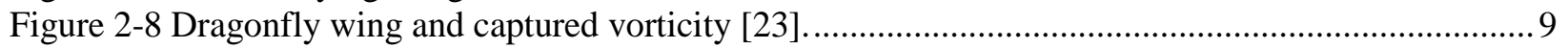

Figure 2-9 Maximum lift-to-drag ratio comparison for smooth and rough airfoils from McMasters [25]. 10

Figure 2-10 Two-dimensional laminar short bubble formation [27] ................................................. 11

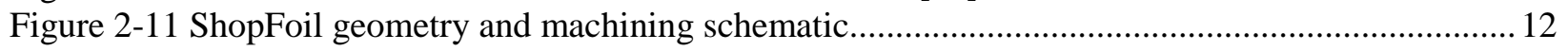

Figure 3-1 Comparison of ShopFoil geometries with an SD6060 ........................................................ 13

Figure 3-2 Two-dimensional C-grid mesh for the SF15126_10 ............................................................ 14

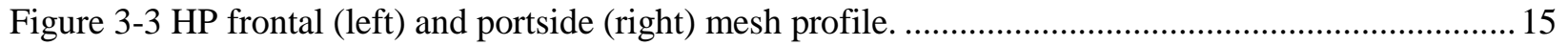

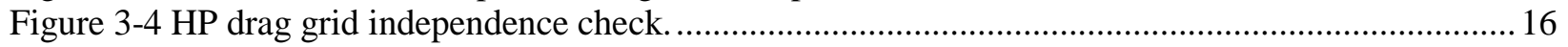

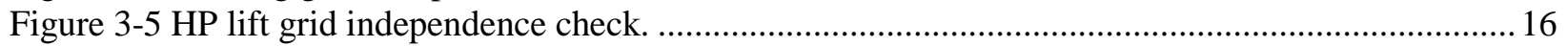

Figure 4-1 SD6060 numerical and experimental lift-curve comparison for $\operatorname{Re}_{\mathrm{c}}=150,000$ (CFD

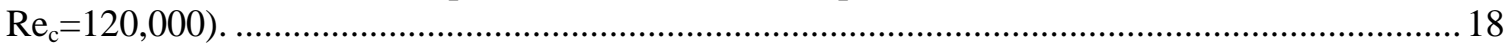

Figure 4-2 Numerical and experimental lift-to-drag comparison at $\operatorname{Re}=120,000(\mathrm{CFD} \operatorname{Re}=150,000) \ldots \ldots .19$

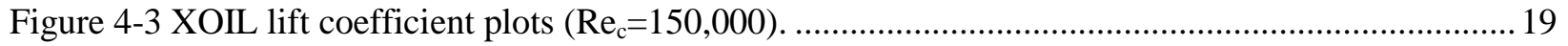

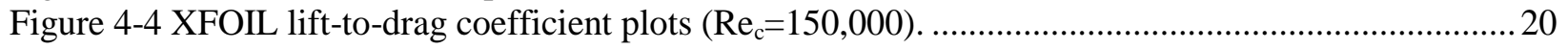

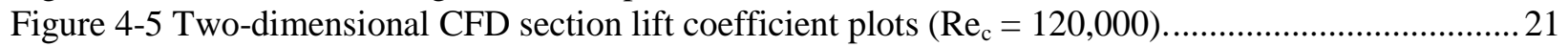

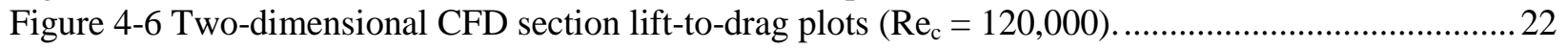

Figure 4-7 Three-dimensional CFD plots of lift coefficient (Coefficients are body-referenced and

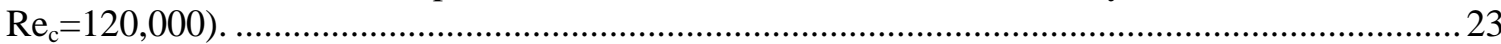

Figure 4-8 Three-dimensional CFD lift-to-drag plots (Coefficients are body-referenced and $\mathrm{Re}_{\mathrm{c}}=120,000$ ).

24

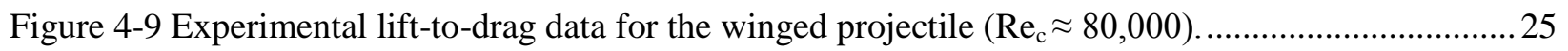

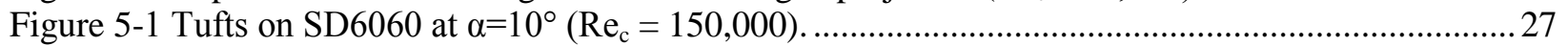

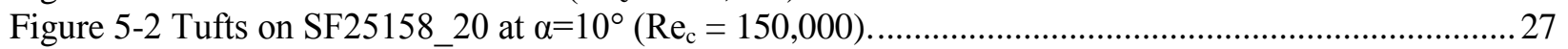

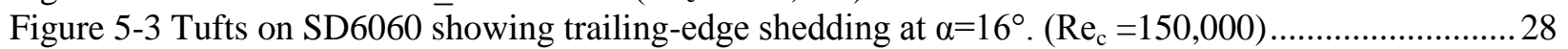

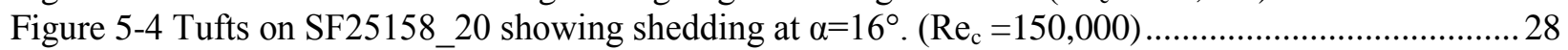

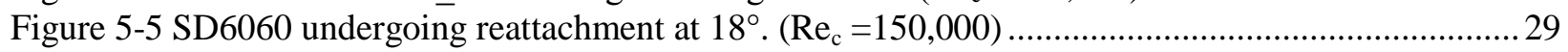

Figure 5-6 SF25158_20 experiencing full separation and recirculation at $18^{\circ}$. $\left(\operatorname{Re}_{\mathrm{c}}=150,000\right) \ldots \ldots \ldots \ldots \ldots . .29$

Figure 6-1 Separation bubble at leading-edge of an SF15126_10, SF25158_20 and SD6060.................. 31

Figure 6-2 Leading edge comparison of SF20158_20 at $12^{\circ}$ (left) and $14^{\circ}$ (right). $\left(\operatorname{Re}_{c}=50,000\right) \ldots \ldots \ldots . . .32$

Figure 6-3 Tufts at the leading-edge of an SF25158_20 aligned with free-stream $\left(\operatorname{Re}_{\mathrm{c}}=150,000\right) \ldots \ldots \ldots 33$

Figure 6-4 Tufts indicating trailing edge separation on a SF25158_20 at $10^{\circ}\left(\operatorname{Re}_{\mathrm{c}}=150,000\right) \ldots \ldots \ldots \ldots . . . .34$

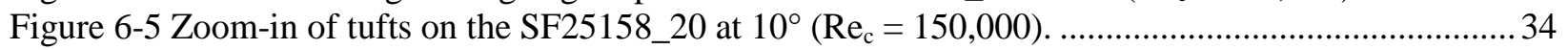

Figure 6-6 Two-dimensional velocity vector plots for SF25158_20 at $10^{\circ}\left(\operatorname{Re}_{\mathrm{c}}=120,000\right) \ldots \ldots \ldots \ldots \ldots \ldots . . . .35$

Figure 6-7 Two-dimensional velocity vector plots of the trailing-edge of the SF25158_20 at $10^{\circ}\left(\operatorname{Re}_{\mathrm{c}}=\right.$

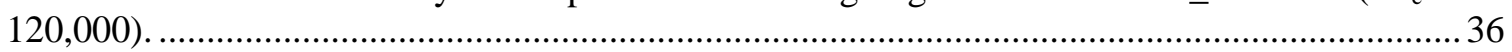

Figure 6-8 Two-dimensional velocity vector plot of the $S F 25158 \_20$ at $12^{\circ}\left(\operatorname{Re}_{\mathrm{c}}=120,000\right) \ldots \ldots \ldots \ldots \ldots . . . .37$

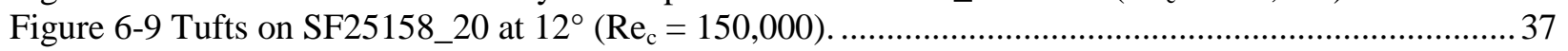

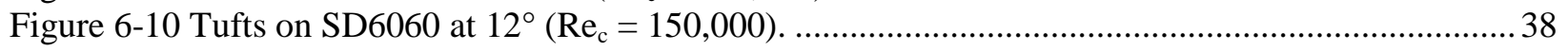

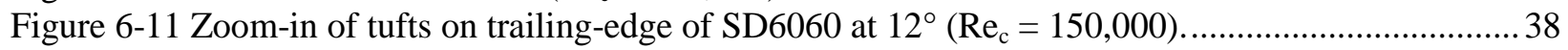


Figure 6-12 Two-dimensional velocity vectors of the $\operatorname{SD6060}$ at $12^{\circ}\left(\operatorname{Re}_{\mathrm{c}}=120,000\right)$.

Figure 6-13 Zoom-in of velocity vectors at the trailing-edge of the SD6060 at $12^{\circ}\left(\operatorname{Re}_{\mathrm{c}}=120,000\right) \ldots \ldots . .39$

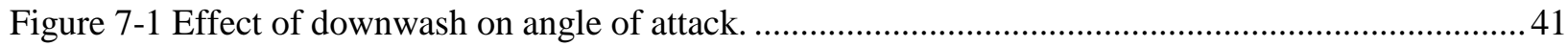

Figure 7-2 Downwash profiles for assorted wing planforms [33].................................................. 42

Figure 7-3 Oil flow of stall progression for a finite, rectangular Clark Y-14 planform of AR $=3.5, \alpha=$

$22.8^{\circ}, \mathrm{Re}_{\mathrm{c}}=245,000$. (Photograph by Allen E. Winkelmann, University of Maryland.)

Figure 7-4 Velocity magnitude contour plots of the SD6060 (left) and SF25158_20 (right) along the span for $\alpha=11.6^{\circ}$ showing reduced separation near the tip. These plots begin with the inboard location (top) and proceed to the tip (bottom). [Note: a visual comparison of the wake profiles of

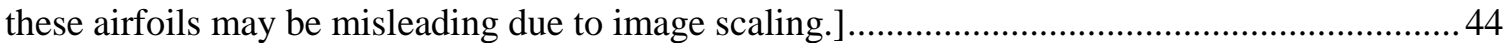

Figure 7-5 Spanwise velocity magnitude contour for SF25158_20 at $11.6^{\circ}\left(\operatorname{Re}_{\mathrm{c}}=120,000\right) \ldots \ldots \ldots \ldots \ldots \ldots . .45$

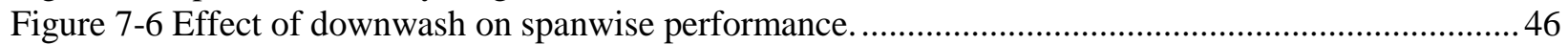

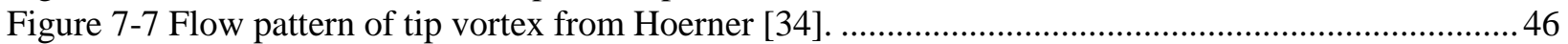

Figure 7-8 Vortex core locations for various tip contours from Hoerner [34] .......................................47

Figure 7-9 Streamlines of 'tip vortex' and inboard flow at wing tip of an SF25158_20. $0^{\circ}$ body angle. 9.5

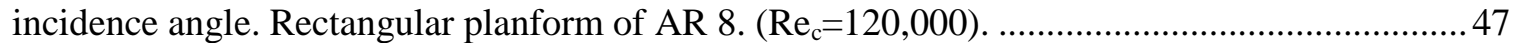

Figure 7-10 Planform-based vortex generators from Pearcey [39]....................................................... 48

Figure 7-11 Contours of velocity magnitudes at successive chordwise locations near tip of SF25158_20 at

$\alpha=11.6^{\circ}$. The images display spanwise slices from the mid-chord to the trailing edge...............49

Figure 7-12 Velocity magnitude contours for cross-sections within the highlighted zone at the wing tips from Figure 7-11. Velocity scale is from $0 \mathrm{~m} / \mathrm{s}$ (blue) to the free stream velocity of $60 \mathrm{~m} / \mathrm{s}$ (green) to $135 \mathrm{~m} / \mathrm{s}$ (red).

Figure 7-13 Two-dimensional velocity contour sections at $-4^{\circ},-6^{\circ}$ and $0^{\circ}\left(\operatorname{Re}_{\mathrm{c}}=120,000\right)$. (Velocity in $\mathrm{m} / \mathrm{s})$

Figure 7-14 Velocity component contours at tip of $S F 25158 \_20$ at $11.6^{\circ}\left(\operatorname{Re}_{c}=120,000\right)$. Streamwise $x-$ component (Left); Spanwise z-component (Right). (Velocity in $\mathrm{m} / \mathrm{s}$ )

Figure 7-15 Wing-tip cross-section contours of z-velocity at $11.6^{\circ}$ showing interaction between the 'tip vortex' and the wing surface $\left(\mathrm{Re}_{\mathrm{c}}=120,000\right)$. Blue contours are pointing into the page, red are coming out of the page. (Velocity in $\mathrm{m} / \mathrm{s}$ ).

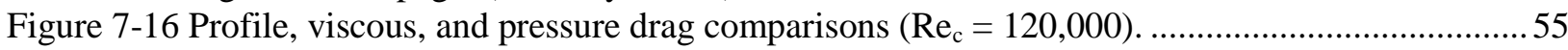




\section{List of Symbols}

$\begin{array}{ll}\text { Symbol } & \text { Description } \\ \mathrm{AR} & \text { Aspect Ratio } \\ \mathrm{c}_{\mathrm{d}} & \text { Section drag coefficient } \\ \mathrm{c}_{1} & \text { Section lift coefficient } \\ \mathrm{C}_{\mathrm{D}} & \text { Hybrid projectile drag coefficient } \\ \mathrm{C}_{\mathrm{L}} & \text { Hybrid projectile lift coefficient } \\ \mathrm{d} & \text { Sectional drag } \\ \mathrm{D} & \text { Hybrid projectile drag coefficient } \\ \mathrm{l} & \text { Sectional lift } \\ \mathrm{L} & \text { Hybrid projectile lift coefficient } \\ & \\ \mathrm{Re}_{\mathrm{Re}} & \text { Reynolds number } \\ \mathrm{Re} & \text { Chord-based Reynolds number } \\ \mathrm{SST} & \text { Shear-stress-transform } \\ \mathrm{U}_{\infty} & \text { Free stream velocity } \\ \mathrm{V} & \text { Free stream velocity } \\ \mathrm{w}(\mathrm{y}) & \text { Downwash velocity at span location y } \\ \mathrm{y}+ & \text { Non-dimensional first cell wall height } \\ & \\ \alpha & \\ \alpha_{\mathrm{eff}} & \text { Angle of attack } \\ \alpha_{\mathrm{i}} & \text { Effective angle of attack } \\ \beta & \text { Induced angle of attack } \\ \Gamma(\mathrm{y}) & \text { ShopFoil surface angle } \\ \rho_{\infty} & \text { Circulation at span location y } \\ & \text { Density of free stream } \\ \end{array}$




\section{Chapter 1 Introduction}

The development of unmanned aerial vehicles (UAVs) has received increasing attention as the realization of expanded applications and more complex capabilities have pushed this technology into potential commercial, while still used mainly in defensive roles. Although in research phases, a subclass of UAVs, micro air vehicles (MAVs), have the potential to be employed in more diverse situations due to their small size, which may allow reduced detectability and greater maneuverability. The trade-off, from an engineering perspective, is operation at low Reynolds numbers and typically required high efficiencies due to space and weight limitations, which are vital for endurance. A particular type of MAV, a Hybrid Projectile (HP), combines the characteristics of a ballistic object and a lift-generating aircraft. Externally, an HP is configured as conventional ammunition to enable launching from available weapons platforms, while interior and exterior modifications allow deployment of remote or on-board controllable lifting surfaces to facilitate navigation and extend range [1]. Such a design may be used for precisely delivering explosive payloads and for reconnaissance with the added possibility of mission abort capabilities, as shown in Figure 1-1.

\subsection{Motivation}

While work to date on Hybrid Projectiles has been somewhat limited, there has been some previous work on the design of stowable vehicles and of wing deployment mechanisms [2][3]. The following discussion examines the deliberations made in pursuance of the development of an HP, in particular, as such development relates to deriving an effective wing planform. It is at first beneficial to recognize the role which the HP is expected to fill. In this case it is one of a short-range, disposable platform operating at low Reynolds numbers, but having the maximum feasible range, endurance, and payload capacity.

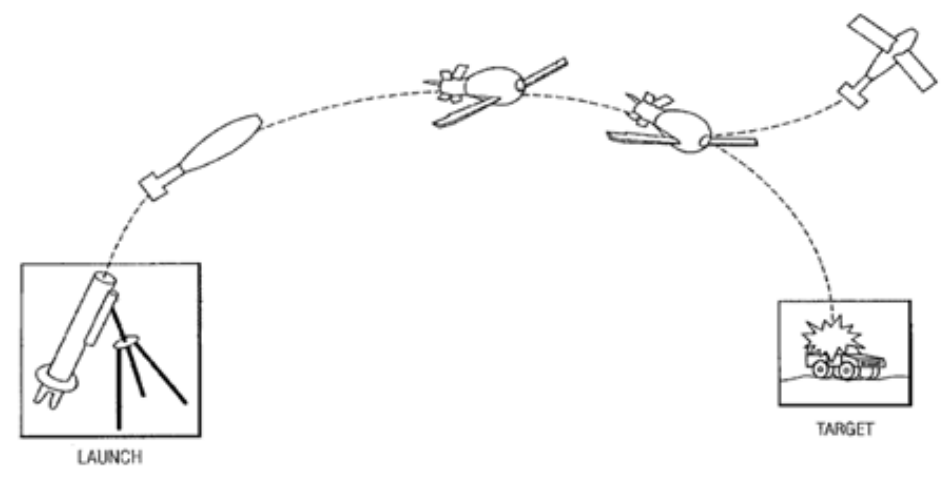

Figure 1-1 Possible HP mission profile [1]. 
A joint consideration of these factors reveals the aerodynamic contrasts between it and traditional winged aircraft. Whereas wing planform and cross-section efficiencies are typically vital to the long-term utility of commercial and military aircraft, including most MAVs and long-range guided weapons systems, the success of the HP may not depend on such high levels of optimized performance. As a disposable platform, the HP carries with it only what it is required to accurately reach relatively close targets. While budget and time costs of airfoil optimization are generally not significant in aircraft development, this process may delay proof of concept design, and depending on operating conditions and fabrication limitations, could prove impractical. An example of this impracticality is the laminar-flow airfoil which was optimized to eliminate drag due to trailing-edge separation. The P-51 Mustang became the first real-world application for this airfoil, but never reached its optimum potential because the manufacturing methods and real-world environment did not meet the precise and polished conditions of the laboratory [4].

From the perspective of structural design the HP wing is restricted by at least three main factors: vehicle size, operating environments, and manufacturing cost and complexity. Due to the small size of the HP platform, traditional rib and spar construction would be tedious and cost prohibitive. Additionally, for the unpredictable scenarios to which it may be subjected, anisotropic materials such as printed plastics and composites might be vulnerable to failure from wing flutter, cracking, have low impact resistance, increased fabrication costs, and require specialized fabrication equipment. Isotropic materials such as Aluminum, however, are readily available, inexpensive, durable, and can easily be machined or formed using standard milling or bending equipment.

With the aerodynamic and structural requirements in mind, prototype development began with an attempt to machine a fully contoured Selig-Donovan low Reynolds number SD6060 airfoil [5][6] from stock Aluminum. This airfoil was initially chosen as it was designed for operation at low Reynolds number and as demonstrated consistent performance over a range of Reynolds numbers from 50,000 to 300,000 . However, difficulties in programming a 3-axis mill to provide the accuracy necessary to reproduce this cross-section proved to be tedious and time-consuming. A reconsideration of the aerodynamic requirements led to the reasoning that approximating an airfoil shape using faceted surfaces may be sufficient to meet the desired range specifications. Further, doing so would enable easy, costeffective machining and reduce the time to testing. 
Although it is possible to reach high levels of machining precision, it comes at a cost. A precision contoured airfoil may be excessive for the HP specifications. Even if precision could be guaranteed, the example of the P-51 illustrates the potential disconnect between design, full-scale manufacturing limitations, and real-world application. Because a faceted airfoil considers fabrication and design upfront, the need for expensive tooling, machining, and systems integration may be mitigated. Benefits of a faceted structure would be lower production costs; faster production rates; increased wing ruggedness (i.e., milling allows for the use of isotropic materials, printed plastics, metals, as well as composite layups are anisotropic and have greater potential for catastrophic failure, microcracking, etc.); reduced facility requirements (e.g., faceted airfoils can be produced on manual, 3-axis mills); enabling manufacturing in make-shift, resource-limited environments. Aside from potential manufacturing benefits, faceted sections may provide increased wing volume in larger scale designs for greater storage capabilities. Due to the increased cross-sectional area over airfoils of the same thickness and chord, they may also provide greater resistance to flutter and bending.

\subsection{Research Objective}

While faceted-type airfoils have been used previously and studied in very limited roles (see Chapter 2), the viability of faceted airfoils for use on a Hybrid Projectile and at the expected Reynolds number range is unexplored. This thesis presents the design and aerodynamic testing of a class of faceted airfoils named ShopFoils. The purpose of this study was to determine whether these airfoils could adequately meet the required glide ratio of $4.5: 1$ for the HP platform, thus providing a rugged and easily machined wing with the desired performance. Additionally, the goal was to understand the flow characteristics of faceted airfoils at low Reynolds number. In order to determine the effectiveness of the ShopFoils various 2D and 3D numerical methods are required as well as wind tunnel tests. Specifically, the objectives of this research are:

1. To rapidly test variants of the ShopFoil class in order to determine whether one of acceptable predicted performance can be created.

2. To apply these airfoils to a typical HP platform and numerically estimate their performance in simulated flight and compare that performance relative to the SD6060.

3. To gain an understanding of the realistic flow characteristics of faceted airfoils at low Reynolds numbers as well as for qualitative validation of numerical results. 
These objectives were accomplished by first using XFOIL, a two-dimensional subsonic airfoil design program, to generate initial rough data regarding the potential for the proposed ShopFoil designs without expending excessive computational resources and time. Second, the variants that were predicted to be the most successful were then tested in three-dimensional computational fluid dynamics (CFD) simulations to provide closer estimates of their real-world applied performance and comparison with the performance of the SD6060 airfoil. Lastly, wind-tunnel flow visualization tests using tufts were conducted for a qualitative comparison of numerical data to reveal the causes of the ShopFoils' performance. 


\section{Chapter 2 Background}

This section provides some history of various previous airfoil developments, including the use of faceted airfoils, discusses some for the key concepts behind low Reynolds number aerodynamics, and presents the concept of ShopFoil.

\subsection{Airfoil History}

Modern airfoil development began in the 1800's as individuals such as Otto Lilienthal and Horatio F. Phillips derived their initial designs by studying the shape of the avian wing. It was reasoned that camber contributed to the efficient generation of lift, but due to the lack of a standardized airfoil theory the design process was relatively laborious and empirical. Phillips referred to his airfoils as 'doubled-surfaced' airfoils, shown in Figure 2-1, as the upper surface was designed with greater curvature on the top than the bottom to produce a region of lower pressure [7]. The airfoils developed by Phillips are predecessors to those used by the Wright Brothers.

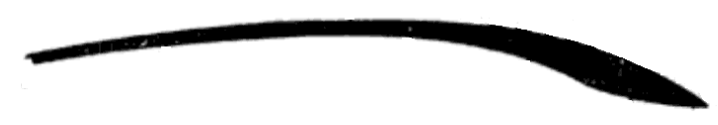

Figure 2-1 Phillips's first cambered airfoil, patented in 1891 (Edited).

In 1912, the Russian Mathematician, Nikolai Joukowsky made a significant contribution to airfoil development by relating lift to circulation and proceedingly using a conformal mapping, referred to as the Joukowsky Transformation, to map the streamlines from an imposed flow around a rotating cylinder to the profile of an elongated airfoil cross-section. This shape is presented in Figure 2-2, and is known as the Joukowsky Airfoil and represents one of the first implementations of 'indirect' airfoil design.

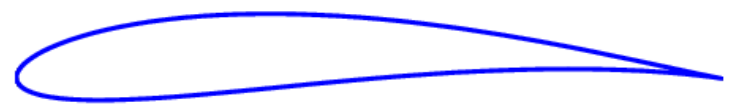

Figure 2-2 Typical Joukowsy Airfoil. 
By World War I airfoils, such as the Gottingen 398 in Figure 2-3 and the Clark Y, had evolved with all the commonly recognizable contours: rounded leading-edge, camber, thickness distribution, and a sharp trailing edge. These two airfoils would later influence the thickness distribution of the NACA series [8][9].

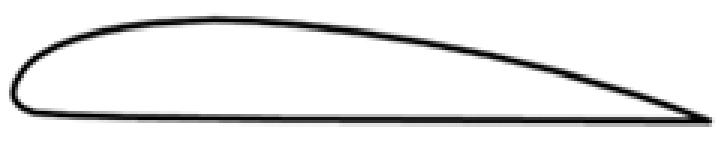

Figure 2-3 Gottingen 398.

The development of 'thin airfoil theory' by M. Munk [10] in the early 1920s and further contributions from Herman Glauert, et al. brought about a more systematic approach to wing design [9]. Thin airfoil geometry could now be determined inversely from the required lift by calculating the net circulation around a representative mean-line. Examples include the M Sections, in honor of Munk, and some of the early NACA series, an example of the four digit series is provided in Figure 2-4. Successive advancements in inverse methods by Eastman Jacobs brought optimized laminar-flow airfoils to prominence at NACA [11] by calculating the precise pressure-gradient required to maximize laminar flow over the wing chord and backing-out the geometry from which it resulted; an inversion of Theodore Theodorsen's direct potential-flow method for an arbitrary shape [12].

Figure 2-4 NACA 2412.

Rather than pursue the development of a new class of airfoils Richard Eppler sought to enable design through better numerical methods while working in cooperation with NASA in the 70s [13]. The result was the versatile airfoil design and analysis code PROFIL capable of both direct and inverse design methods. There are now a multitude of computational tools capable of achieving accurate results both directly and inversely, while the quantity and role of application-specific airfoils are too numerous to address in this report. However, it is useful to cover some background on faceted wing-sections. 


\subsection{Faceted Airfoils}

Although faceted airfoils have not been in prominent use, they are not without their own history with both man-made and natural applications. One of the first uses may have been occasionally employed on return boomerangs by the Aborigines and other cultures [14]. For the Reynolds numbers experienced in boomerang flight, blunt leading and trailing edges provide beneficial flight characteristics: a blunt leading edge provides slower spin and shorter hover, whereas blunt trailing edges provide rounder flight [15]. While faceted surfaces were likely not used for the longer-range hunting boomerangs, this case provides an example of their satisfactory performance for a specialized, short-range application. The basic design of the return boomerang can be seen in Figure 2-5; note the upper surface and trailing edge.

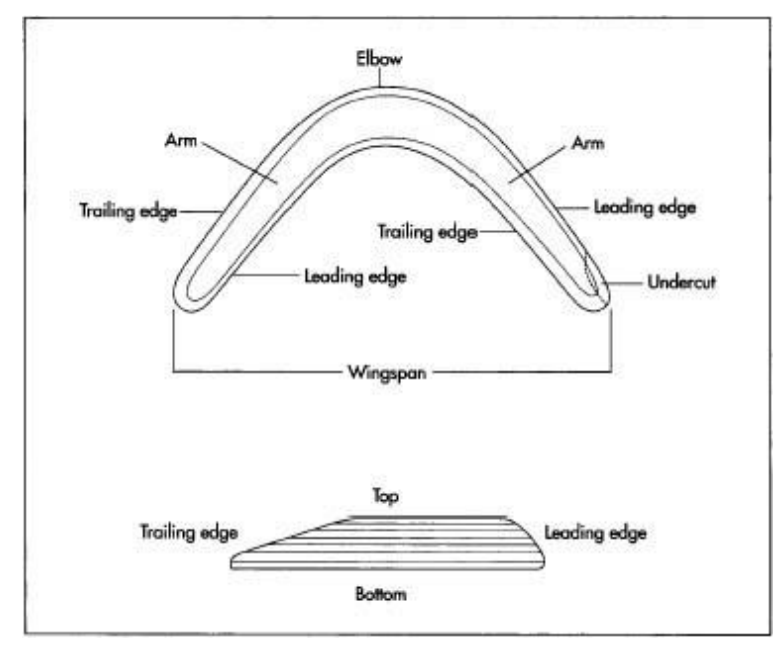

Figure 2-5 Faceted upper surface and trailing edge of a return boomerang.

Another low Reynolds number use of faceted-type airfoil surfaces was discovered by Richard Kline and Floyd Fogleman when developed a series of "stepped" airfoils in the late 60s and 70s, shown in Figure 2-6, called Kline-Fogleman (KF) airfoils [16][17]. The cross-sections consist of a single or series of flat surfaces joined by 'steps' in the spanwise direction with the aim of trapping a vortex or series of vortices to maintain flow attachment over a wide range of attack angles. Initially designed for paper airplanes, studies conducted at NASA in 1974 revealed poor lift-to-drag ratio and that the KF airfoils offered no advantages over conventional airfoils [18]. These airfoils have since found use in Foam RC aircraft along with claims ranging from improved stall performance to increased hover abilities by hobbyists. 


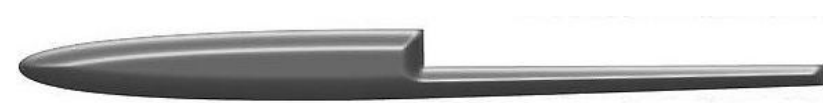

KFm-2

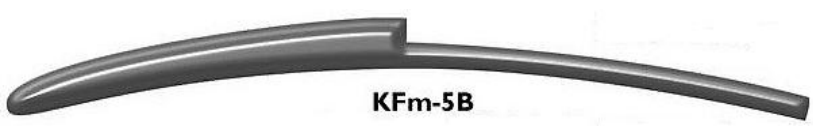

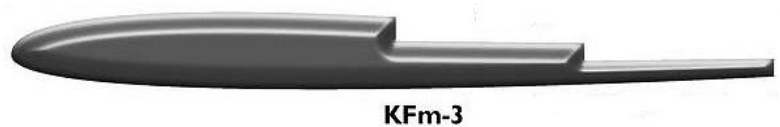

KFm-3

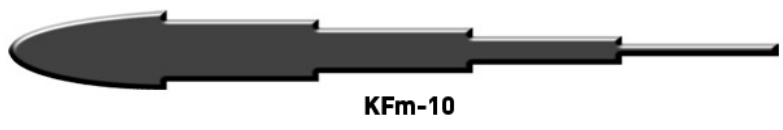

Figure 2-6 Kline-Fogleman Airfoils.

Perhaps the most immediately recognizable aircraft which employed faceted wings is the F-117 Night Hawk shown in Figure 2-7. The use was not to achieve particular aerodynamic or structural performance but rather to reduce radar cross-section (i.e., for stealth). While it is possible that faceted surfaces hindered the aerodynamic efficiency in this application, the design was successful, and capable of achieving high subsonic velocities (approx. $680 \mathrm{mph}$ ) and conducting long-range missions. Additionally, the F-117 demonstrates that rough geometries can generate battle-functional planforms, even at higher Reynolds numbers.

An additional application of these planforms is the Facetmobile, also shown in Figure 2-7. This aircraft employs an inverse Zimmerman design, which is a mix between a lifting body and a flying wing. Built by Barnaby Wainfan, Rick Dean, and Lynne Wainfan, the Facetmobile utilizes a sharp leading-edge with the aim of generating and "trapping" a vortex on the planar leading-edge surface with the intent of increasing lift coefficient through the phenomenon of 'vortex lift' [19]. Further benefits are addressed in a report prepared for NASA which concluded: faceted, low aspect ratio aircraft can outperform conventional aircraft: yielding high manufacturability and time savings; allowing reduced costs by eliminating the need for expensive and specialized tooling; and may be produced at half the cost of other methods [19] [20]. This supports the ShopFoil concept discussed in Section 1.2.

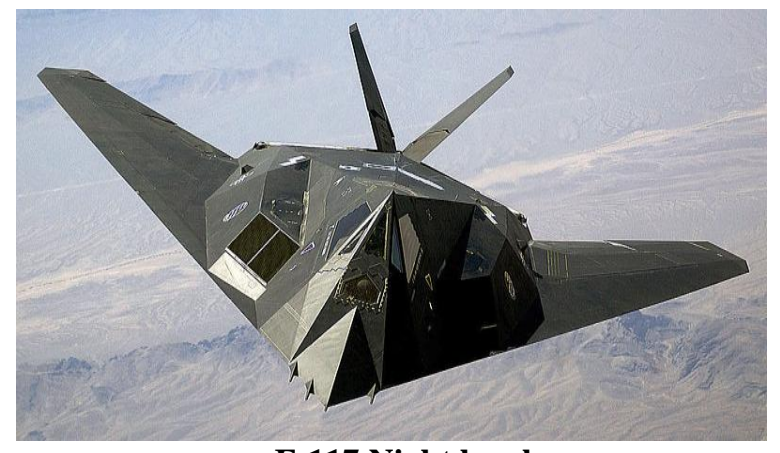

F-117 Night hawk

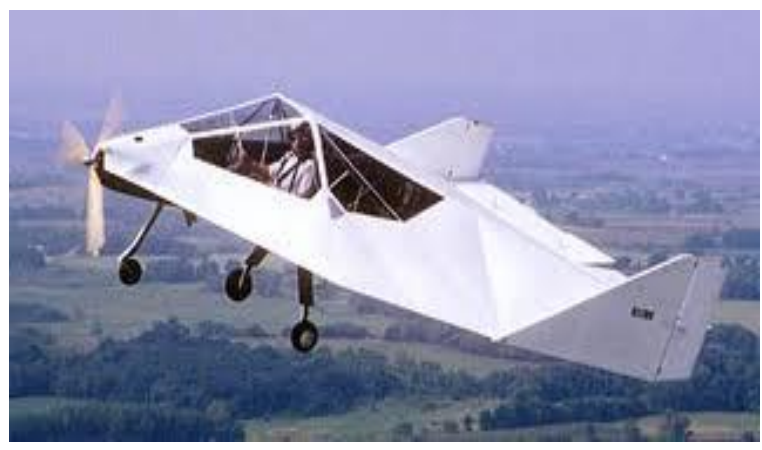

Facetmobile

Figure 2-7 Faceted flying-wing aircraft. 
Having addressed some of the man-made uses of faceted wings, it is noted that nature has also taken advantage of these planforms, although creating more complex designs than the man made counterparts discussed above. More commonly referred to as a corrugated structure, the cross-section of a dragonfly wing is comprised of multiple faceted surfaces, which offers structural rigidity and low Reynolds number $\left(1000 \leq \mathrm{Re}_{\mathrm{c}} \leq 10,000\right)$ aerodynamic benefits compared to streamlined airfoils. The corrugation of these wings form spanwise channels which trap vortices; aiding in the prevention of flow separation and stall by acting as "turbulators" for quickly transitioning the boundary layer from laminar to turbulent [21]. These vortices also alter the effective profile depending on angle of attack, in effect, providing a morphing airfoil [22]. Refer to Figure 2-8 for flow visualization displaying these effects.

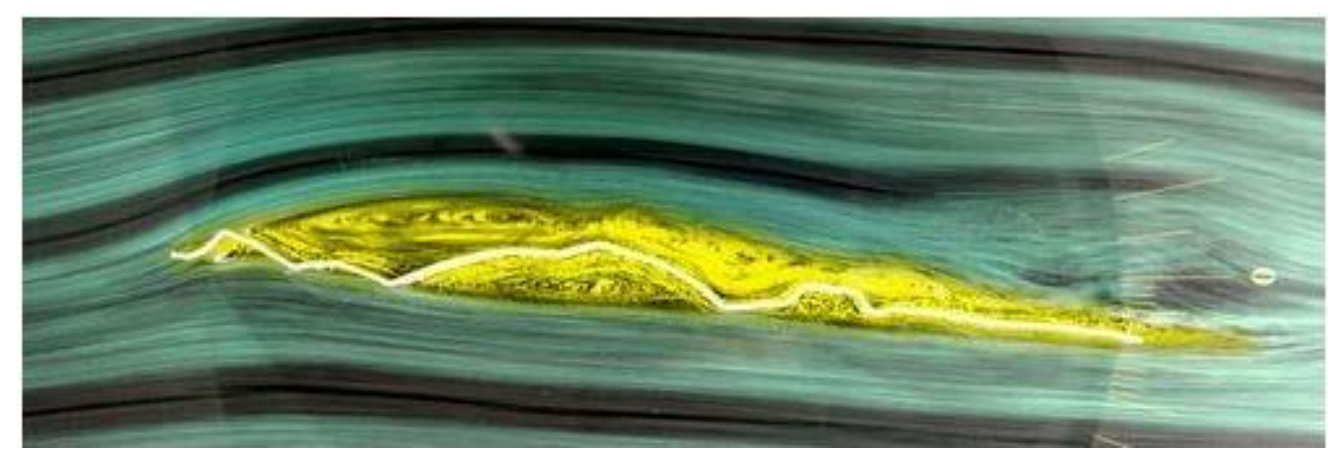

Figure 2-8 Dragonfly wing and captured vorticity [23].

These examples demonstrate that faceted surfaces, if properly designed, are capable of performing over a wide-range of Reynolds numbers, while potentially delivering unique properties such as specialized aerodynamic benefits, structural rigidity, cost-savings, simplified and expedited manufacturing, and although not the goal of ShopFoil even radar avoidance (assuming properly calculated geometries). 


\subsection{Low Reynolds Aerodynamics}

The optimal shape of an airfoil depends largely on the required size and speed at which it travels and is referred to as scale effect, and is characterized by the Reynolds number, $\operatorname{Re}$ (a function of $\rho, u, c$, and $\mu$ ). Low Reynolds number airfoils generally operate in the range of $10^{2}$ to $10^{5}$, and include insects, birds, model aircraft, UAVs and MAVs. Larger aircraft such as human powered, general aviation, commercial transports, and military aircraft operate in the range of $10^{5}$ to $10^{9}$. The differences in airfoil shape at low Reynolds number are most notable in a transition region of about 70,000 which is shown in Figure 2-9. Below this the most effective airfoils have rough surfaces such as the case of the Dragonfly, but above this value of the Reynolds number smooth airfoils begin to dominate lift-to-drag efficiencies [24].

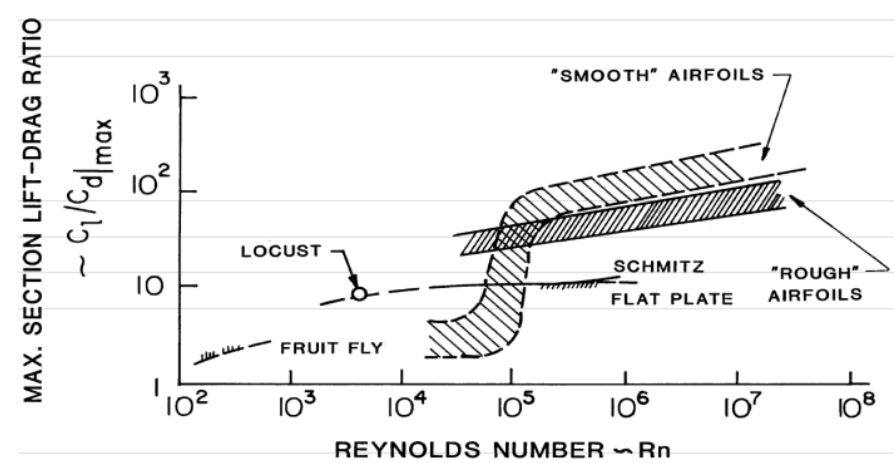

Figure 2-9 Maximum lift-to-drag ratio comparison for smooth and rough airfoils from McMasters [25].

The area of low Reynolds number aerodynamics is still relatively unexplored as most modern research and application has focused on large-scale wings; however, UAV and MAV development have recently increased efforts in this area. Despite the need for further investigation there a few key aspects of flows in this regime which are of important consideration. Because the low Reynolds number flow regime occurs prior to the point of the laminar-turbulent transition, the flows are most often laminar.

For Reynolds numbers below 30,000 airfoils at relatively high angles of attack have a problem overcoming the adverse pressure gradients as their boundary layers remain laminar. Between $\operatorname{Re}_{\mathrm{c}}$ numbers 30,000 and 70,000 airfoils can be subject to laminar separation, and transitioning of the shear layer to turbulent flow; thus hysteresis is common, but this effect is affected by airfoil thickness. However, at the lower end of this range the chord length is generally not long enough for attachment to occur due to the tendency of the detached shear layer to remain laminar. Above the transition region of $\operatorname{Re}_{c}=70,000$ and below 200,000, laminar flow can be maintained over a large percentage of the chord, bringing performance improvement [26]. 
The formation of laminar bubbles, such as that shown schematically in Figure 2-10, is dependent on the chord length of the airfoil where the resulting bubble may be long or short. At higher Reynolds numbers, on the order of $10^{5}$, a short bubble is likely to form at lower angles of attack and for lower numbers in this region a long bubble can form. However a short bubble can 'burst' to become a long bubble if the pressure gradient becomes too strong [24].

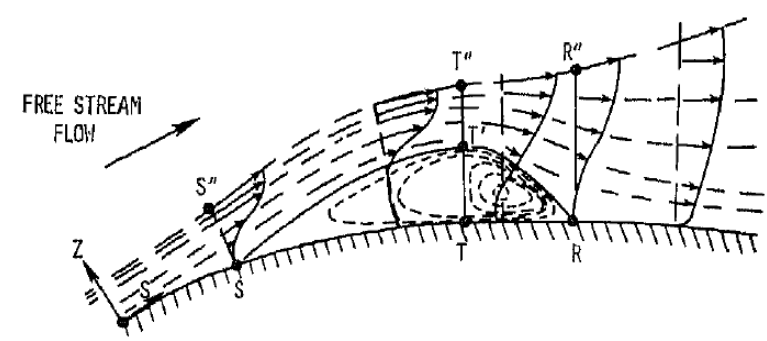

Figure 2-10 Two-dimensional laminar short bubble formation [27].

\subsection{ShopFoil Generation and Design}

In order to illustrate a basic type of form which a faceted airfoil may take, consider the geometry for the ShopFoil family of sections as illustrated in Figure 2-11. The stock which forms the wing blank starts as a rectangular cross-section (denoted by the black border), and the leading-edge is then rounded using a standard concave mill end. The remaining steps of the ShopFoil shape are based on relatively simple cuts which can be made using a 3-axis mill. The top surface of the geometry consists of surfaces one, two, three, and four. Surface one, two, and three are produced through three angled face cuts, represented by $\beta_{1}, \beta_{2}$, and $\beta_{3}$. The $y_{3}$ parameter fixes the height of surface three, fully defining the geometry. It is the distance from the intersection of the line collinear with surface 3 and the right vertical edge to the midpoint of that edge. Surface four is the remaining edge of the initial rectangular geometry after the angled face cuts are made. The resulting vertices connecting each surface are then moderately filleted. Each ShopFoil is named according to a specified pattern to code in the particular geometry. For example, if $\beta_{1}=15^{\circ}, \beta_{2}=12^{\circ}, \beta_{3}=6^{\circ}$, and $y_{3}=10$, the naming convention would follow as SF15126_10. The beta angles and $\mathrm{y}_{3}$ set the rules for each instance of the ShopFoil class. 

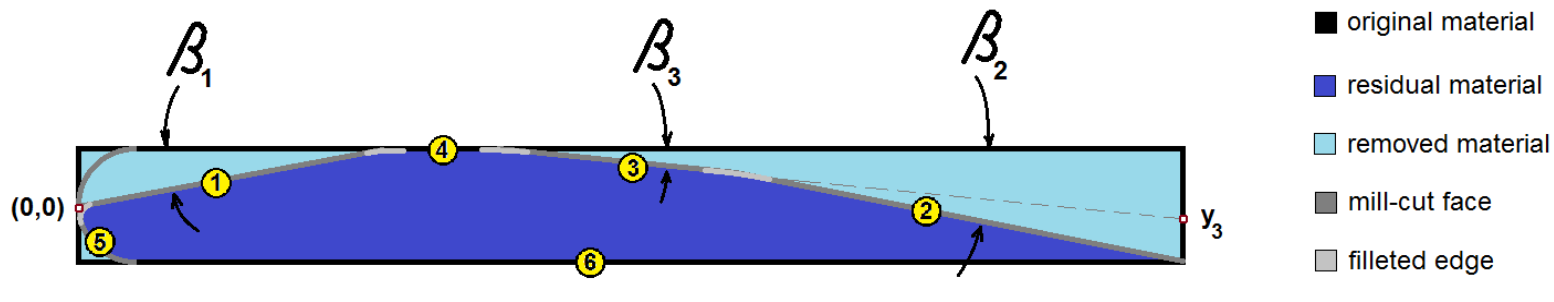

Figure 2-11 ShopFoil geometry and machining schematic.

A potential area for improvement in this method lies in the geometrical rules presented in the ShopFoil schematic. Airfoil coordinates have widely been presented non-dimensionally so that they can be applied to varying wing size; this is not the case in this instance. Although it seemed reasonable at the time, the $y_{3}$ rule which fixes the height of the tangent line to the $\beta_{3}$ surface also fixes the chord length of the airfoil, requiring adjustment for alternate sizing. This naming convention has been altered in recent work to make the geometry nondimensional. 


\section{Chapter 3 Procedure}

The following chapter covers the details regarding the procedure for producing satisfactory ShopFoils. However, a general overview of the numerical testing proceeded can be expressed as follows: 1.) establishing a sample of varying ShopFoil planforms; 2.) rapidly generating 2D data using XFOIL; 3.) assuming acceptable preliminary performance, test a selection of the exceptional planforms in 2D CFD to verify XFOIL data; and 4.) simulating flight in 3D CFD.

\subsection{Test Matrix}

A ShopFoil test matrix was chosen according to the rules defined in Section 2.4 with varying angles: $\beta_{1}=15^{\circ}-25^{\circ}$ in $5^{\circ}$ increments, $\beta_{2}=12^{\circ}$ or $15^{\circ}$, and $\beta_{3}=6^{\circ}$ or $8^{\circ}$, providing twenty-six initial geometries. Coordinates for each of these twenty-six sections were generated using an Excel spreadsheet which distributed points along each of the rule-derived airfoil geometries. Utilizing a test matrix allows for a streamlined trial and error means to determine which angle combinations are most effective. This is not unlike the basic premise behind an evolutionary algorithm, where the population field is initialized with a varying set of characteristics. Here those characteristics are the surface angles.

However, this process does not involve the iterative optimization in such algorithms as the goal is only to find an airfoil which is "good enough". Figure 3-1 provides both a comparison between some of the cross-sections which will be discussed in this thesis as well as two examples of ShopFoil geometries which have been produced: the SD6060 formed the basis or performance target for our present design, the SF0012 is one of the first ShopFoil designs, it is a symmetric section which was rejected due to poor performance, and the SF15126_10 and SF25158_25 which are further discussed in this paper.

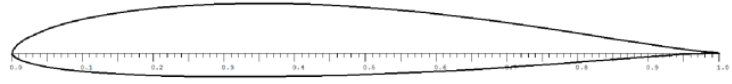

SD6060

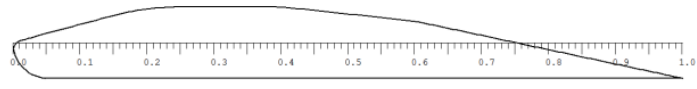

SF15126_10

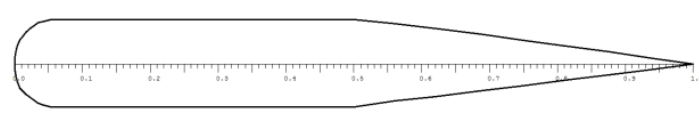

SF0012

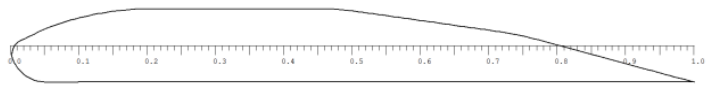

SF25158_25

Figure 3-1 Comparison of ShopFoil geometries with an SD6060. 


\subsection{XFOIL}

The initial field of twenty-six ShopFoils were narrowed to six using XFOIL [28], a subsonic airfoil design program. XFOIL was chosen because it is capable of both inviscid and viscous formulations as well as estimating boundary-layer growth and separation; it allows for quick run-times; and is available under the GNU General Public License -it is "free". To understand why a program such as XFOIL is well-suited for this stage of the ShopFoil design process, consider that we were able to process angle of attack sweeps for twenty-six geometries at four Reynolds numbers on a single desktop computer in only a few hours, while obtaining reasonably accurate lift prediction and qualitative comparisons. The same process with CFD would likely have taken weeks or months.

To the author's knowledge, there are no previous examples of XFOIL or numerical methods being employed to determine the flow characteristics for the types of rough, geometrical approximations under consideration. For this reason, the role of XFOIL was limited to providing rapid and useful qualitative data by which the ShopFoil test matrix could be reduced, 1/d and $c_{1 \alpha}$ plots for Re of 50,000, 100,000, 150,000, and 200,000 for the estimation stall angle and zero-lift angle. Additionally, the SD6060 was chosen as a target performance standard due to its known consistency across the same low Re range. Six of the airfoils which could most closely met this target were down-selected.

\subsection{Two-dimensional CFD}

With the field of ShopFoils narrowed, testing continued with two-dimensional CFD for further comparison with the SD6060. The implementation of CFD was intended to provide greater flow detail, potentially revealing any exceptionally negative or positive flow characteristic effects which XFOIL was incapable of providing. The process for conducting the two-dimensional computational test began with the generation of a C-grid mesh in ICEM, of which an example is provided in Figure 3-2 below. Initially, a coarse mesh consisting of approximately 10,000 nodes was created followed by a grid independence check.

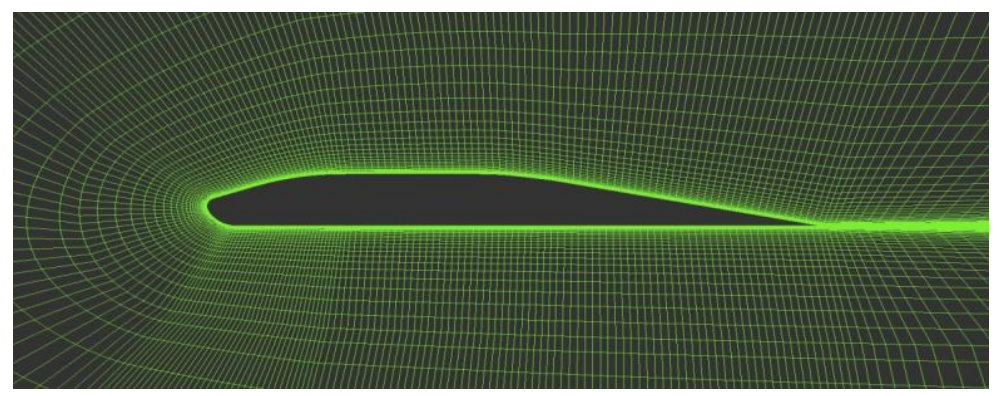

Figure 3-2 Two-dimensional C-grid mesh for the SF15126_10. 
The meshes, having a $y^{+}<1$, were loaded into Fluent's two-dimensional solver and run with the $\kappa \omega$-SST turbulence model with default settings aside from the setting for the turbulence intensity and viscosity ratio which was set to $1 \%$. Previous application of the $\kappa \omega$-SST model at this Re range has demonstrated accurate lift prediction at the possible expense of drag over-estimation [2]. The tests were conducted at a Reynolds number of 120,000 for angle of attack increments of $2^{\circ}$ between $-6^{\circ}$ and $12^{\circ}$.

\subsection{Three-dimensional CFD}

With the two-dimensional numerical tests suggesting satisfactory potential, three-dimensional modeling was sought to estimate how the most promising ShopFoils would perform in application. The candidates were out-fitted on a tail-less low-Re, HP platform with a rectangular wing with a bodyinclusive aspect ratio of 8 , having no taper or sweep, zero twist, an incidence angle of approximately $9.5^{\circ}$, and dihedral angle of $6^{\circ}$. The body diameter is a relatively large percentage of the span. It was modeled in ICEM using a fully-structured mesh consisting of 2.5 million nodes, shown in Figure 3-3, with all surfaces of the hybrid model having a $y+<1$. As in the two-dimensional tests, the $\kappa \omega-S S T$ model [29] was used and the meshes were then run with Fluent's three-dimensional solver at Re of 120,000 over body angle of attack range from $-6^{\circ}$ to $4^{\circ}$ in $2^{\circ}$ increments. Prior to any extensive testing, a grid independence study was conducted, the results of which are provided in Figure 3-4 and Figure 3-5.
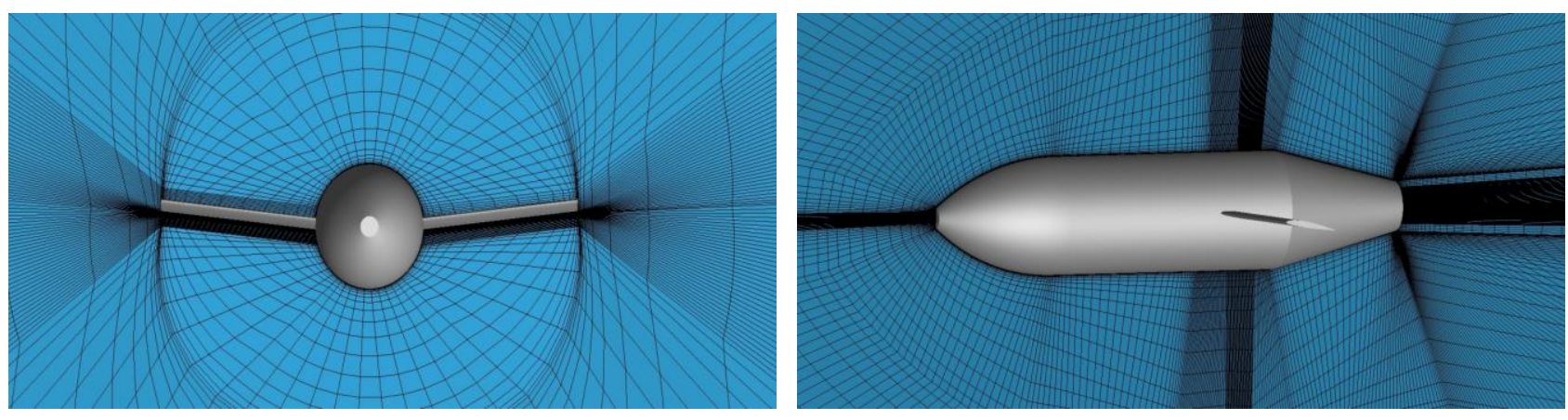

Figure 3-3 HP frontal (left) and portside (right) mesh profile. 


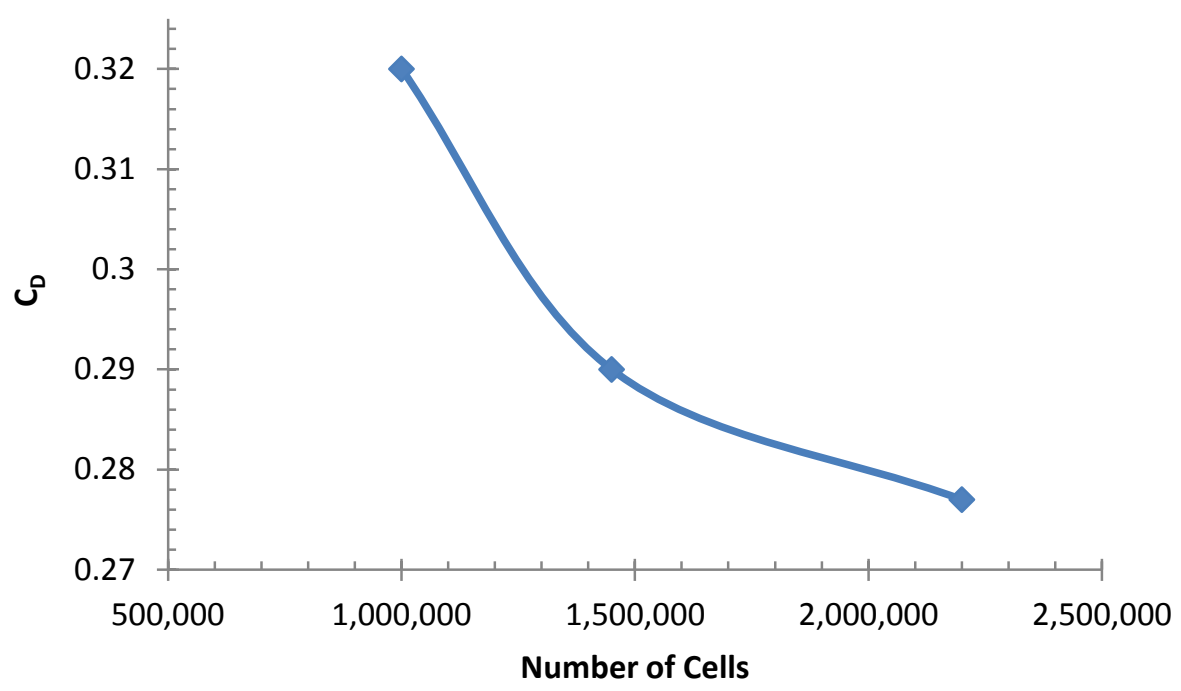

Figure 3-4 HP drag grid independence check.

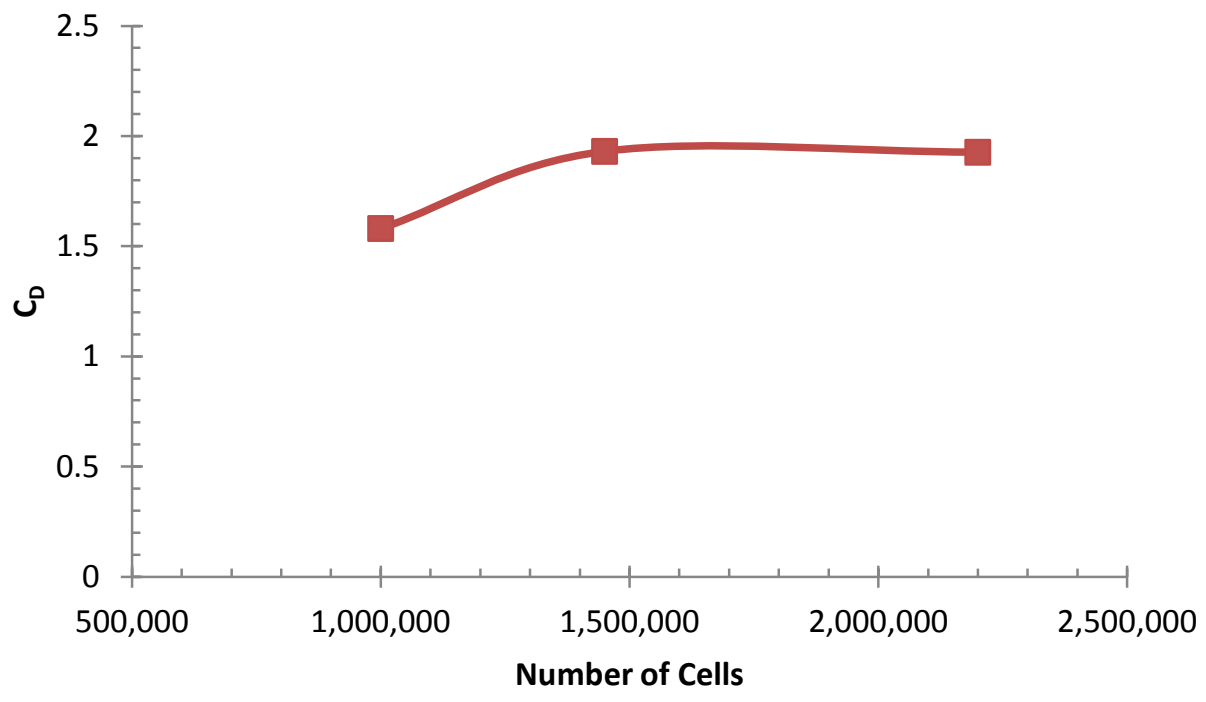

Figure 3-5 HP lift grid independence check. 


\subsection{ShopFoil Flow Visualization}

As a continuation of the ShopFoil research project, wind tunnel tests of a hybrid model discussed in Chapter 4 indicated that a particular section actually outperformed the SD6060, in addition to the CFD simulation results supporting the comparable performance of the these two sections. Within the scope of the work presented in this paper, a continuation of these experiments proceeded in the form of further wind tunnel tests to provide qualitative information on the flow characteristics of the SD6060 and ShopFoil wing-sections in the form of flow visualization utilizing tufts.

Four sections were chosen for comparison: SD6060, SF20158_20, SF25128_20, and SF25128_25. These airfoils were fabricated with foam and fiberglass composite. A foam core was cut from SpyderFoam using a hotwire foam cutter and laser-cut wing-section templates. These templates were replicas of the geometry from the SolidWorks design files. The foam cores were then covered in fiberglass/resin composite and end-plates attached on either side to maintain two-dimensional flow. These models were not measured for accuracy; however, they are cut from exact replicas of the CAD models and were determined to be sufficient for the purposes of these experiments.

A side-mount sting was constructed which allowed angle-of-attack adjustments in $1^{\circ}$ increments. The sections were mounted on the sting and adjusted to zero according to the wind-tunnel horizontal. A chordwise row of vertical and horizontal tufts were placed at the half-span of each section. Each of these sections were tested at Reynolds numbers of 50,000, 100,000, and 150,000 for angles of attack from $0^{\circ}$ to $20^{\circ}$ with a camera mounted in the horizontal position to capture tuft motion for each case. A flow angle measurement was not taken for the purposes of this comparative study; however, the WVU subsonic wind tunnel can see down-drafts up to $2^{\circ}$ or $3^{\circ}$. 


\section{Chapter 4 Numerical Results}

The results presented here cover the computational methods employed on the ShopFoil while comparing the performance of ShopFoil geometry with the SD6060 airfoil. In addition, three dimensional CFD and experimental data are presented and discussed.

\subsection{XFOIL}

The role of the XFOIL code was to determine whether the faceted airfoil design was worth pursuing on the HP platform and to narrow the twenty-four ShopFoil candidates according to predicted performance for further testing. However, XFOIL has been shown to be capable of relatively accurate lift predictions at this Reynolds number. XFOIL data and two-dimensional CFD data using the $\kappa \omega-S S T$ model for the SD6060, collected by the author, is provided in comparison with the experimental data for the SD6060 collected by C. Fox at $\operatorname{Re}_{c}=150,000$ in Figure 4-1 and Figure 4-2. XFOIL's lift prediction is within $5-10 \%$ at higher angles of attack up to $\alpha_{\text {stall }}$ Overall, XFOIL's lift to drag predictions for the SD6060 follow the general trend of the experimental data with some drag under-estimation between $0^{\circ}$ and $7^{\circ}$.

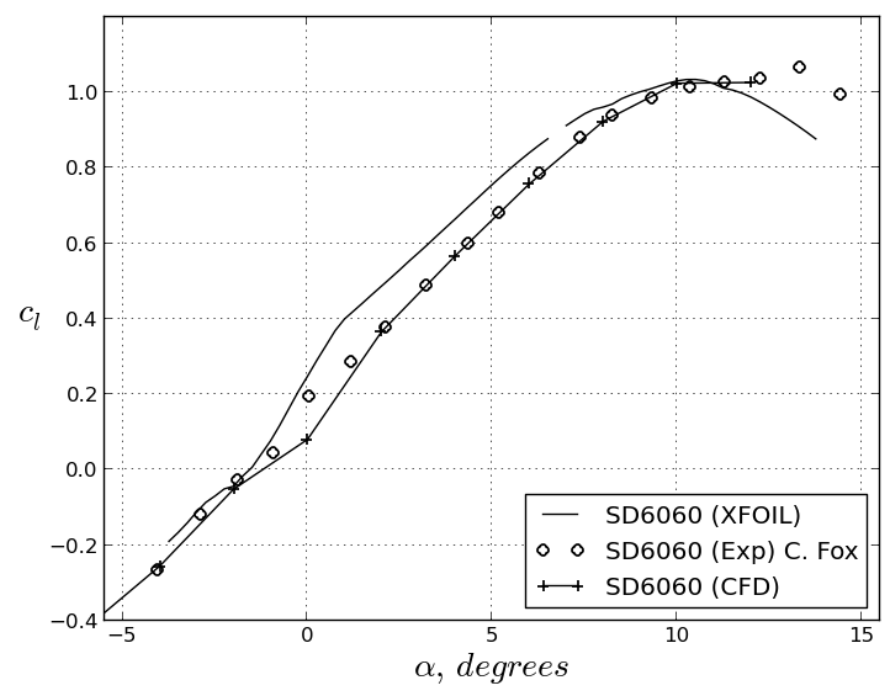

Figure 4-1 SD6060 numerical and experimental lift-curve comparison for $\operatorname{Re}_{\mathrm{c}}=150,000\left(\mathrm{CFD} \mathrm{Re}_{\mathrm{c}}=120,000\right)$. 


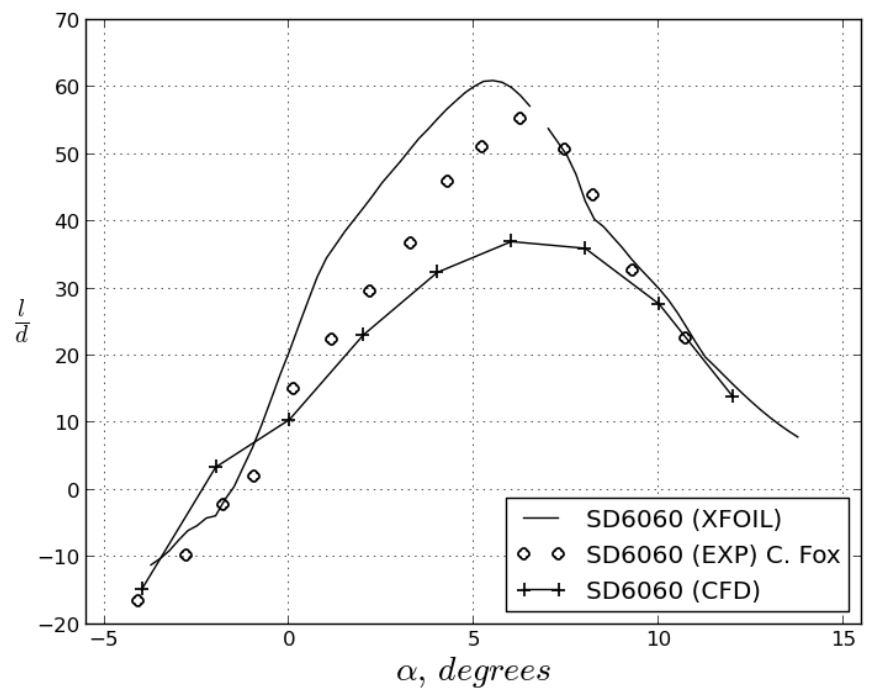

Figure 4-2 Numerical and experimental lift-to-drag comparison at $\operatorname{Re}=120,000(\mathrm{CFD} \operatorname{Re}=150,000)$.

In the plots of Figure 4-3 and Figure 4-4 the performance of an SF25158_20 and an SF15126_10 are compared with the SD6060 from XFOIL data. These two ShopFoils are representative of the two extremes; those which have been omitted performed within the upper bounds of the former and lower bounds of the later. The presentation of the available data at all Reynolds numbers is not of benefit for the purpose of this section, thus only the data at the chord Reynolds of 150,000 is shown.

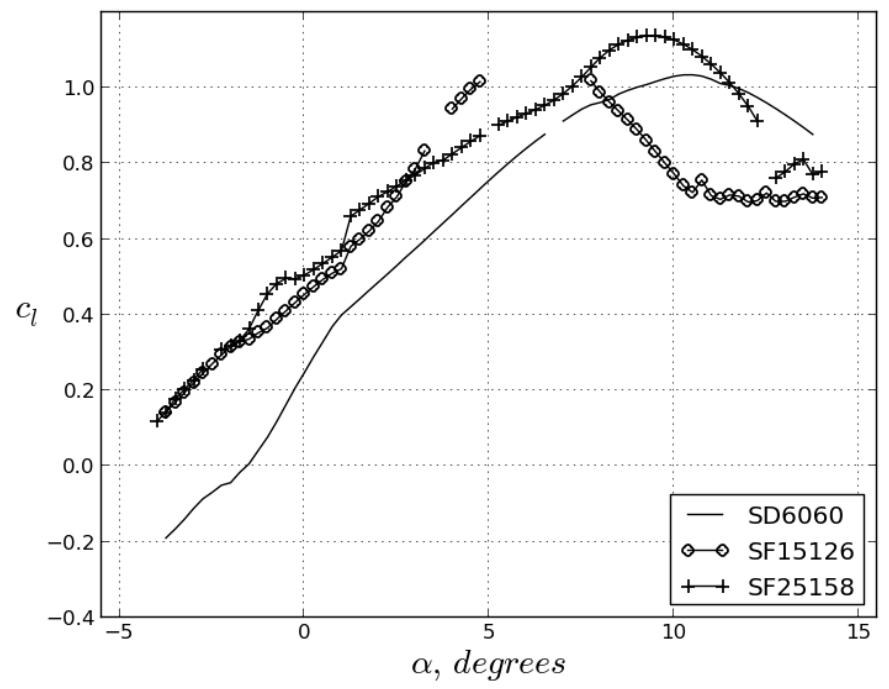

Figure 4-3 XOIL lift coefficient plots $\left(\operatorname{Re}_{\mathrm{c}}=\mathbf{1 5 0 , 0 0 0}\right)$. 


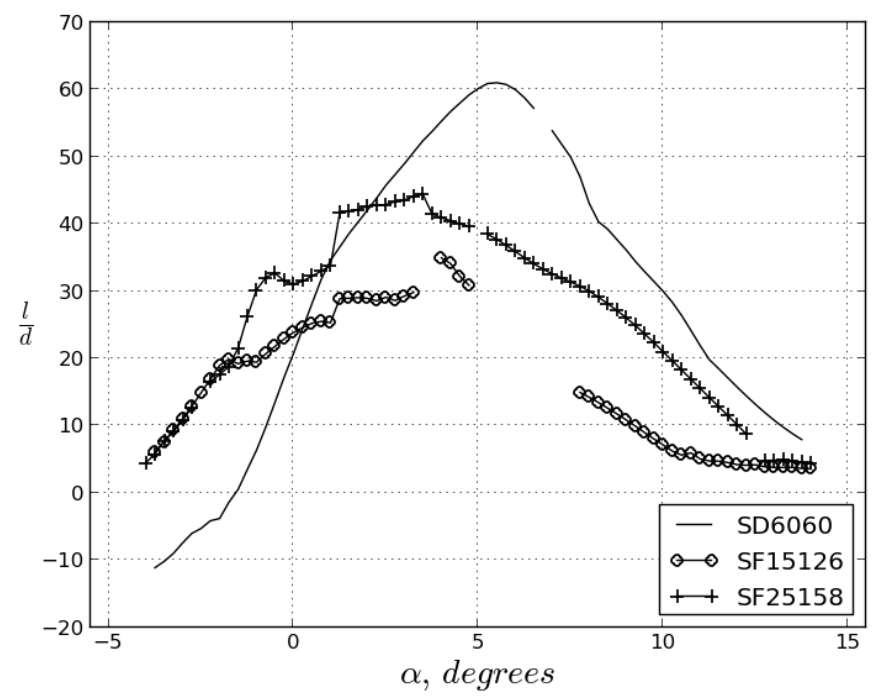

Figure 4-4 XFOIL lift-to-drag coefficient plots $\left(\operatorname{Re}_{\mathrm{c}}=150,000\right)$.

The lower-end ShopFoils represented by the SF15126_10 were marked by convergence problems, such as those from $4^{\circ}$ to $7^{\circ}$, lower stall angles, and relatively poor lift-to-drag performance. Both the discontinuities and 1/d were assumed to be the result of separation issues. The higher-end of the ShopFoils, such as the SF25158_20, were generally "well-behaved" in XFOIL. The lift-curve slope in Figure 4-3 shows a smooth and steady increase in lift which exceeds the lift coefficient of the SD6060 until about $12^{\circ}$. The convergence issues experienced with the SF15126_10 were not present, indicating that separation may be less likely. Further, the lift-to-drag performance shown in Figure 4-4 is closer to the SD6060 which can be attributed to increased lift and lower drag. For example, the maximum lift-todrag predicted for the S25158_20 occurs at approximately $4^{\circ}$ with a value of $1 / \mathrm{d}_{\max }$ approximately 43 , whereas the same performance mark for the SF15126_10 occurs at $4^{\circ}$ with an approximate value of 36. Relative to the $1 / \mathrm{d}_{\max }$ of the SD6060 (approximately 61 at $5.5^{\circ}$ ), this represents a difference of $29 \%$ and $41 \%$ respectively.

There are a couple characteristics of the ShopFoils that are important to take away from these results: peak and low angle-of-attack lift generation exceeds that of the SD6060 (which will be shown to be consistent throughout all numerical testing in subsequent sections); and that while the lift-to-drag performance does not appear at this stage to be equivalent to the SD6060, at lower angles of attack it is within 10-15\%. Based on the results provided by XFOIL five ShopFoils were down-selected for further analysis using CFD: the SF25158_20, SF25128_25, SF25126_10, SF20158_20, and SF20128_25. 


\subsection{Two-Dimensional CFD}

After narrowing the most promising SF candidates with XFOIL, more accurate comparisons with the SD6060 were sought using CFD. Along with the down-selected ShopFoils, a SF15126_10 was chosen in order to provide some idea of XFOIL's ability to qualitatively distinguish between the performance characteristics of these airfoils; it was considered that XFOIL may provide misleading data, potentially due to the limitations of its methods.

Two-dimensional CFD runs for these airfoils were conducted from $-6^{\circ}$ to $12^{\circ}$ in $2^{\circ}$ increments at a Re number of 120,000 (corresponding to a velocity of $60 \mathrm{~m} / \mathrm{s}$ ). To get an idea of the accuracy of the $\kappa \omega$-SST model refer to Figure 4-1 and Figure 4-2. The lift predictions accurately compare with experiment while drag is over-predicted. All the ShopFoils achieve a higher $\mathrm{c}_{1}$ than the SD6060 at lower angels of attack, each demonstrating similar performance over this region. Except for the SF15126_10 and SF20128, the remaining ShopFoils reach their lift peak between $9^{\circ}$ and $10^{\circ}$. The SF25158_20, again, achieves a peak $c_{1}$ of approximately 1.1, while the SF15126_10 doesn't quite reach the above a $c_{1}$ of 1 as XFOIL suggests. Overall, however, this data is consistent with that provided by XFOIL.

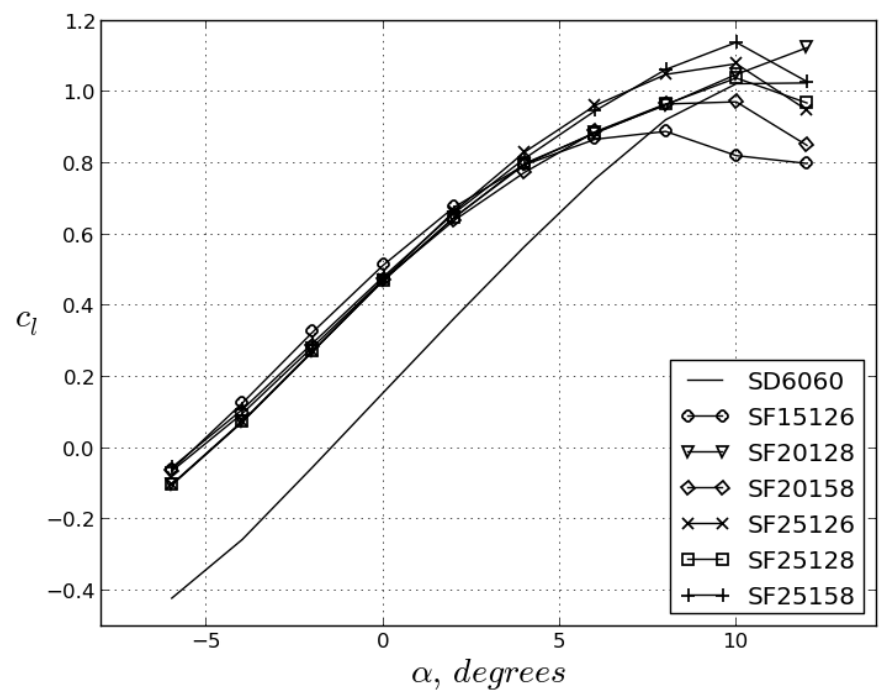

Figure 4-5 Two-dimensional CFD section lift coefficient plots $\left(\operatorname{Re}_{\mathrm{c}}=\mathbf{1 2 0 , 0 0 0}\right)$.

The 1/d plot in Figure 4-6 is also of interest as it suggests similar trends between CFD and the XFOIL results in Figure 4-4. The SF15126_10 is the obvious poor performer reaching a maximum 1/d of 28.5 at around $2^{\circ}$ whereas the best performer among the ShopFoils is predicted to be the SF25126_10 with an 1/d of 35 at twice the angle of attack. The SD6060 for comparison achieves an 1/d of 37, leaving an approximate 5\% difference between it and the SF25126_10. 


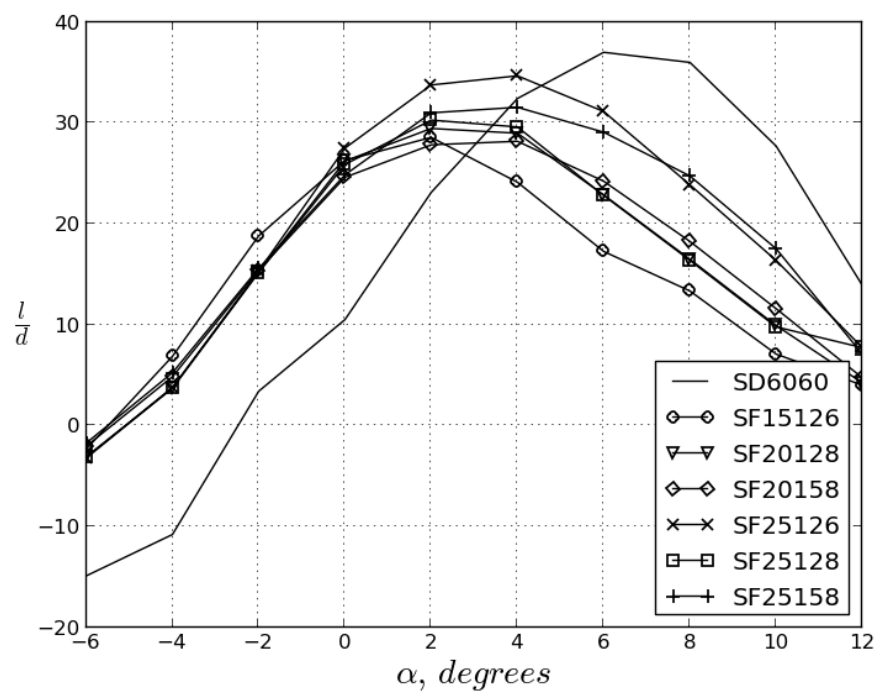

Figure 4-6 Two-dimensional CFD section lift-to-drag plots $\left(\operatorname{Re}_{\mathrm{c}}=120,000\right)$.

Considering the data globally, what becomes obvious is the overall under-performance in $1 / \mathrm{d}$ in comparison to that predicted by XFOIL. The $1 / \mathrm{d}_{\max }$ for the SF25158_20 predicted by XFOIL was 43. The data in Figure 4-6 suggests that both the SF25126_10 and the SD6060 outperform the SF25158_20, but neither achieve an 1/d of 43; the SD6060 certainly does not achieve the XFOIL predicted value of 61, nor even the more conservative experimental values of 57 obtained at $\operatorname{Re}$ of 200,000 and 43 at $\operatorname{Re}$ of 100,000. This isn't surprising, however, since as noted in a previous section, the $\kappa \omega$-SST model has been found to provide accurate lift predictions at the expense of drag over-estimation.

Taking the data as provided requires making the assumption that any potential under-reporting of lift-to-drag affects both the ShopFoils and the SD6060 equally. For the purposes of this study, that assumption was made. The result of this suggests that the top-performing ShopFoils, although having their $1 / d_{\max }$ at a lower angle-of-attack, may be within 5-10\% of the SD6060. 


\subsection{Three-dimensional CFD}

With two-dimensional CFD indicating that ShopFoil potentially performs within $10 \%$ of the SD6060 in reference to L/D, testing proceeded with the tail-less body geometry shown in Figure 3-3 to verify the three-dimensional, low Re flight characteristics of these faceted planforms. Recall that the angle of attack presented in Figure 4-7 is the body-angle, where the wing incidence angle is $9.6^{\circ}$, and that the coefficients present are fuselage-diameter referenced.

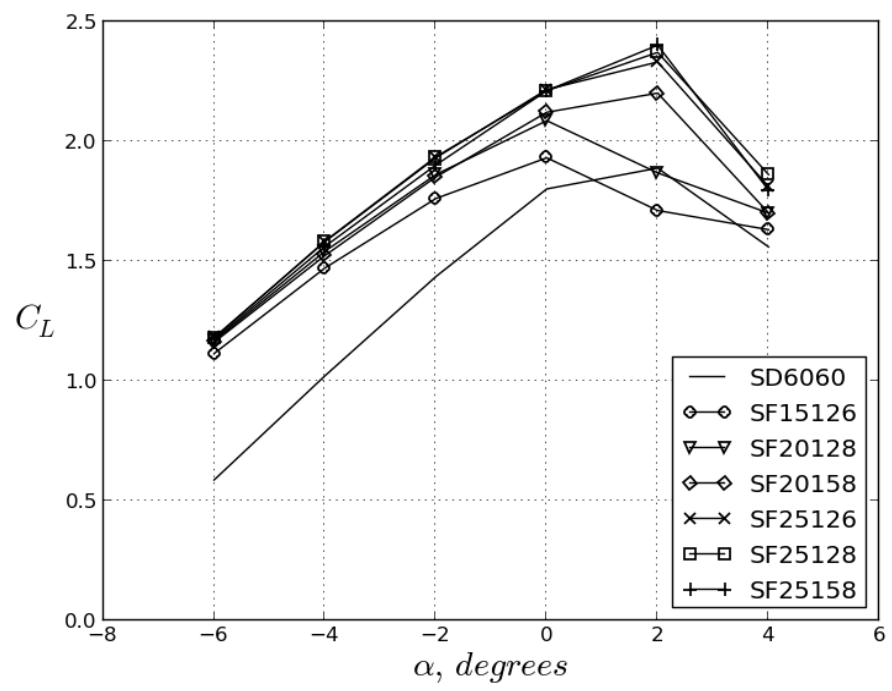

Figure 4-7 Three-dimensional CFD plots of lift coefficient (Coefficients are body-referenced and $\left.\operatorname{Re}_{c}=120,000\right)$.

Considering first the data in Figure 4-7 it can be seen that the ShopFoil geometries show superior performance to the SD6060 in lift generation showing consistent low angle-of-attack performance with the previous XFOIL and two-dimensional CFD data. The lift peak for the SF15126_10 and SF20128 occur at a wing angle of attack corresponding to approximately $9^{\circ}$ with the other sections reaching their maximum lift coefficients at $\alpha=11^{\circ}$. While this data is consistent with previous numerical results from a qualitative perspective, there are noticeable quantitative differences. 


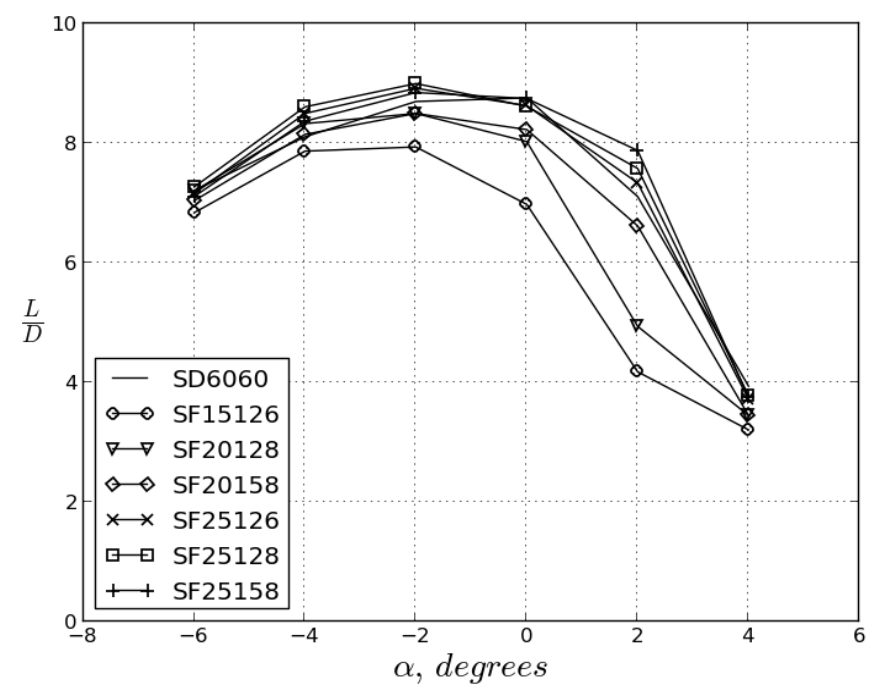

Figure 4-8 Three-dimensional CFD lift-to-drag plots (Coefficients are body-referenced and $R_{c}=120,000$ ).

The lift-to-drag plots in Figure 4-8 suggest that the SF25158_20, SF25128_25, and SF25126_10 perform equivalently or better than the SD6060-equipped body with the SF20158_20 relatively close. According to the three-dimensional data, certain ShopFoil sections could potentially perform similarly to the SD6060 planform within the same angle of attack range. When comparing these results with the XFOIL and two-dimensional CFD results in Figure 4-4 and Figure 4-6, respectively, it is apparent that the data is in contrast as the two-dimensional data suggests that the best ShopFoils are producing $L / \mathrm{D}_{\max }$ within a margin of $15 \%$ but at lower angles-of-attack. It seems as though there is some kind of threedimensional effect which is responsible for this shift in L/D.

\subsection{Experimental Data}

In addition to the three dimensional CFD simulations, experimental data was collected for the same winged-body and wing planform in alternate project using the down-selected ShopFoils [30]. It is noted in Figure 4-9 for comparison with the CFD results and because it served as the impetus for the wind tunnel flow visualization experiments presented in Chapter 5 and Chapter 6. Noting that the Reynolds numbers differ, the L/D plots demonstrate similar behavior to the simulation results. The SF15126 is presented here and has been shown in two-dimensional and three-dimensional numerical testing to be of relatively poor performance compared to the most effective ShopFoil geometries. However, this data suggests it may outperform the SD6060 at low angles of attack, while operating within 10-20\% at higher angles of attack. As is suggested in the data of Figure 4-8, the performance of the SF15126 diverges from the SD6060 around $\alpha=0^{\circ}$. 


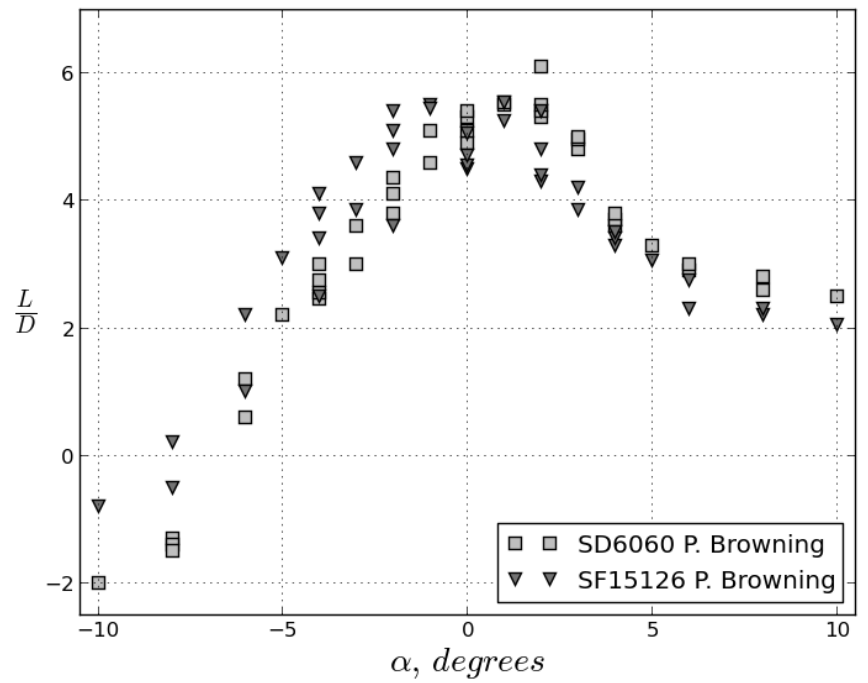

Figure 4-9 Experimental lift-to-drag data for the winged projectile $\left(\operatorname{Re}_{\mathrm{c}} \approx \mathbf{8 0 , 0 0 0}\right)$.

\subsection{Numerical Testing Summary}

The key points that can be taken from the two and three dimensional numerical results are as follows:

- The three numerical approaches consistently indicate that the ShopFoil sections generate more lift than the SD6060, while generally producing more drag at higher angels of attack.

- In three-dimensional simulations, the L/D advantage of the SD6060 is not present in comparison to certain ShopFoils (e.g., SF25158_20). This implies some effect which is not present in two-dimensional flow or possibly the inability of the model to accurately predict the performance of these sections.

- The numerical results seem to suggest that particular ShopFoil designs can perform comparably to the SD6060 at the tested Reynolds number and also meet the glide ratio requirement of 4.5:1.

- Experimental results for the SF15126 are comparable to the three-dimensional simulations. 


\section{Chapter 5 Wind-Tunnel Flow Visualization}

The impetus for the flow visualization experiments stems from the three-dimensional CFD results and experimental lift and drag data presented in Figure 4-9, which indicated that particular ShopFoil sections potentially performed equivalently to the SD6060 and possibly maintained flow attachment at higher angles of attack. Experience would imply that this is an unexpected result due to the fact that the SD6060 has been optimized for performance at these Reynolds numbers, whereas the faceted sections of ShopFoils do not follow traditional airfoil contouring which prevents separation via pressure gradient manipulation. Thus, they would be expected to underperform. The intention here is to determine what mechanism if any may be responsible for the level of performance indicated by experiment and also that which was noted in the three-dimensional lift-to-drag plots of Figure 4-8.

The experimental results discussed here provide qualitative comparisons of the flow characteristics among four airfoils: the SD6060, SF20158_20, SF 25128_25, and SF25158_20. For this section discussion is limited mainly to the SF25158_20 and SD6060 for a Reynolds number of 150,000 and specified angles-of-attack. This was done because there are no significant visible qualitative differences in the fundamental aerodynamic behavior of the various ShopFoils to illustrate, nor many substantive differences between Reynolds numbers. The SF25158_20, however, did maintain flow attachment a degree or two beyond that of the SF20158_20 and the SF25128_25, and as a result appeared to more closely match the characteristics of the SD6060 - which is the justification for the direct comparison in this section.

\subsection{Comparison at $\alpha=10^{\circ}$}

The first comparison begins at $\alpha=10^{\circ}$ as shedding can be visibly seen by the motion of the tufts at the trailing edge of the SD6060 and SF25158_20 in Figure 5-1 and Figure 5-2 respectively, while at lower angles of attack the flow remains attached and there is little to compare. As this is only a qualitative comparison there is no way to definitively state differences in magnitude for the shedding of these airfoils. However, from the images it can be noted that the shedding is beginning at about the two-thirds chord location, perhaps a bit later for the SD6060. In both cases there does not appear to be any indication of separation bubbles formed through reattachment. Neither the vertical nor the more traditionally positioned tufts in Figure 5-2 indicate any phenomenon other than attached stream-wise flow at the leading edge and shedding toward the trailing edge. 


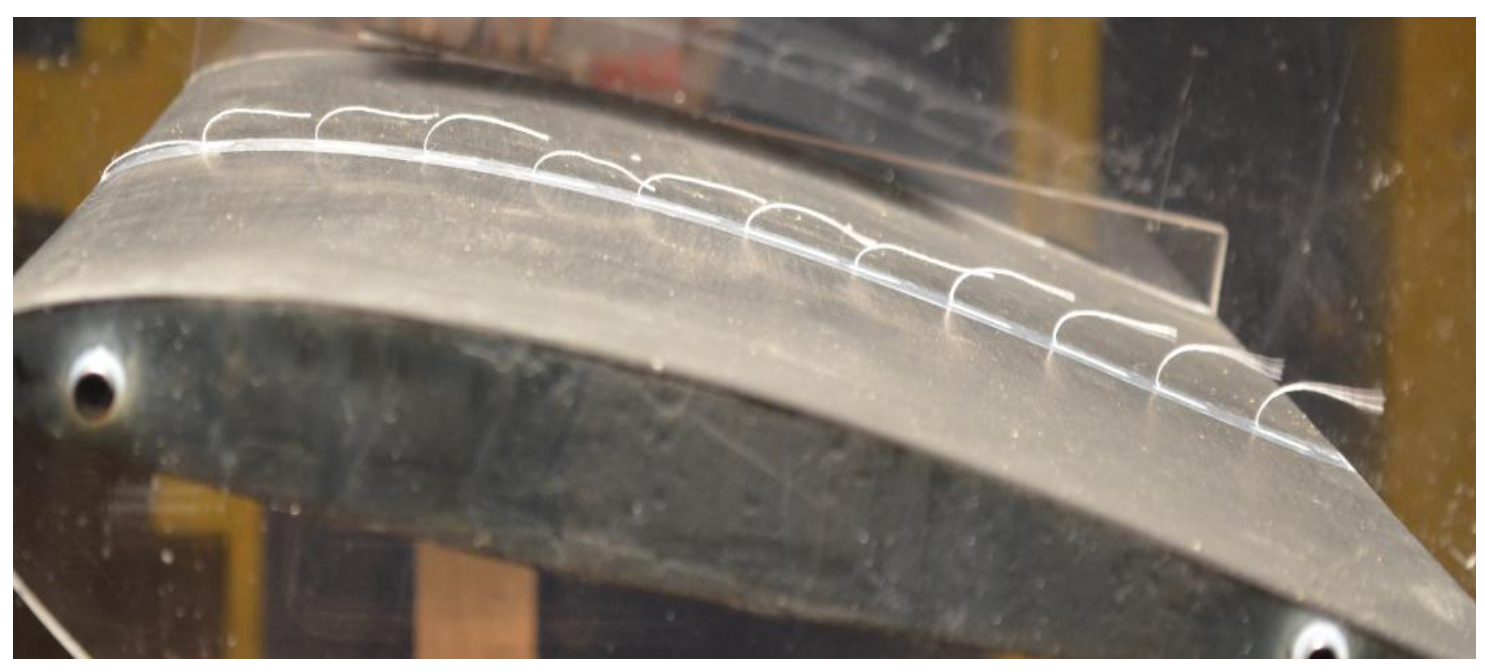

Figure 5-1 Tufts on SD6060 at $\alpha=10^{\circ}\left(\operatorname{Re}_{\mathrm{c}}=150,000\right)$.

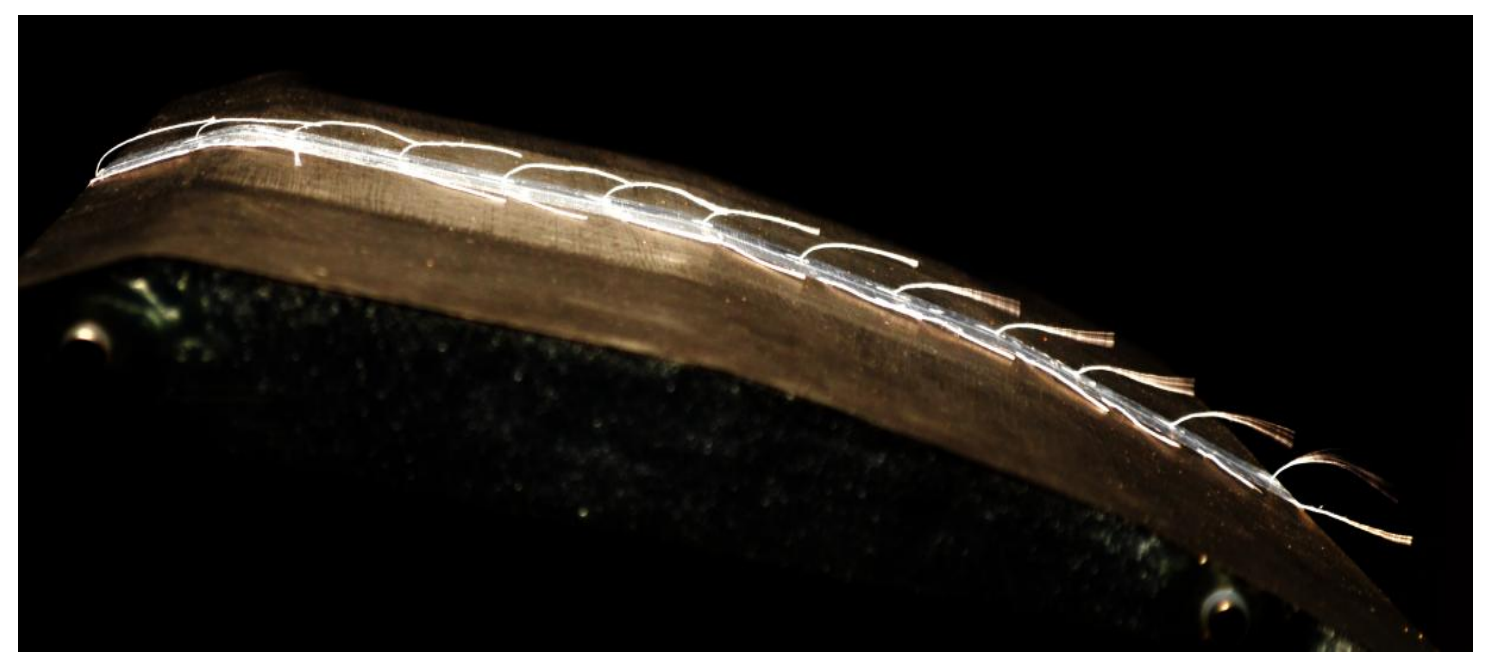

Figure 5-2 Tufts on SF25158_20 at $\alpha=10^{\circ}\left(\operatorname{Re}_{\mathrm{c}}=150,000\right)$.

\subsection{Comparison at $\alpha=16^{\circ}$}

The second comparison is at an angle-of-attack of $16^{\circ}$. Considering the behavior of the tufts in Figure 5-3 and Figure 5-4, the separation has advanced to near the 50\% chord and the shedding is more pronounced. There is no sign of an attached recirculation on either the SD6060 or any of the surfaces of the SF25158_20. The shedding appears to have a dominant streamwise component which may indicate that it is not composed of large-scale laminar eddies as appears to be the predicted state in the CFD. 


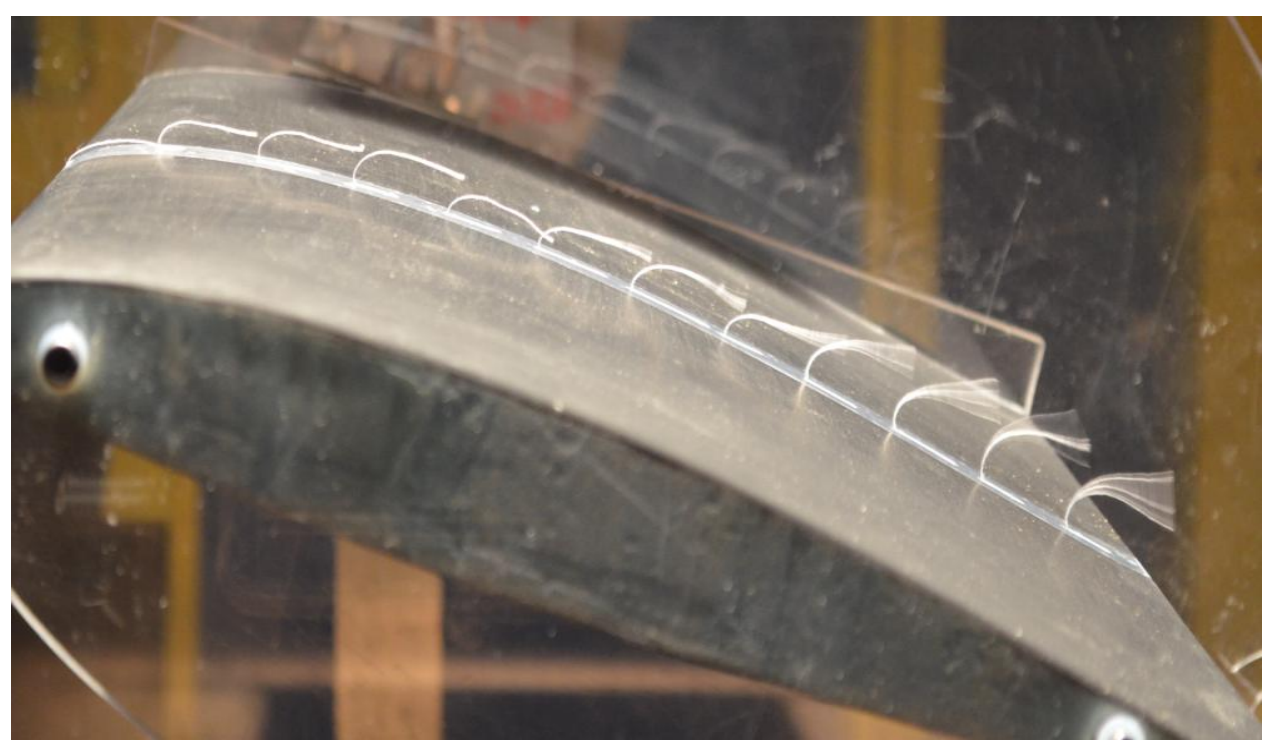

Figure 5-3 Tufts on SD6060 showing trailing-edge shedding at $\alpha=16^{\circ} .\left(\operatorname{Re}_{\mathrm{c}}=150,000\right)$

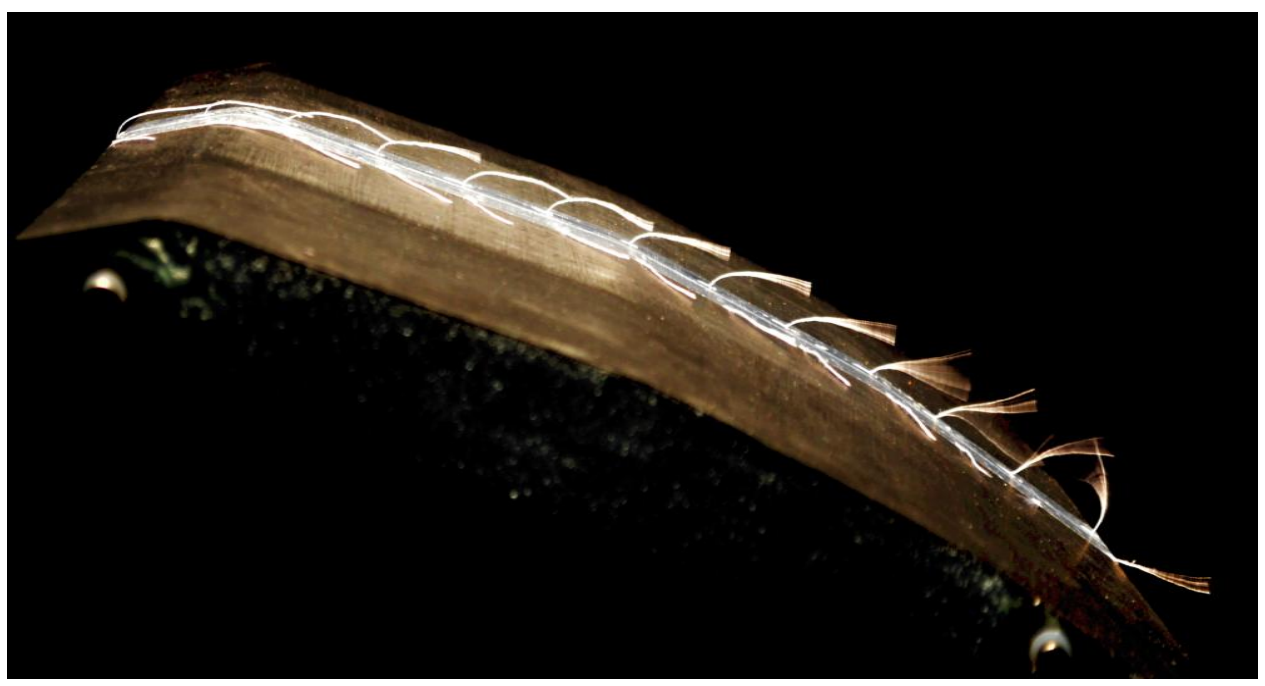

Figure 5-4 Tufts on SF25158_20 showing shedding at $\alpha=16^{\circ} .\left(\operatorname{Re}_{\mathrm{c}}=150,000\right)$

\subsection{Comparison at $\alpha=18^{\circ}$}

Upon reaching eighteen degrees angle-of-attack the flow over the aft portion of the SD6060 detached yet formed a periodically steady long separation bubble as shown in Figure 5-5. This is evidenced by the last three tufts which are aligned upstream. The SF25158_20 experienced full separation and unstable recirculation indicated by the tufts rapidly shifting from stream-wise to upstream direction. 


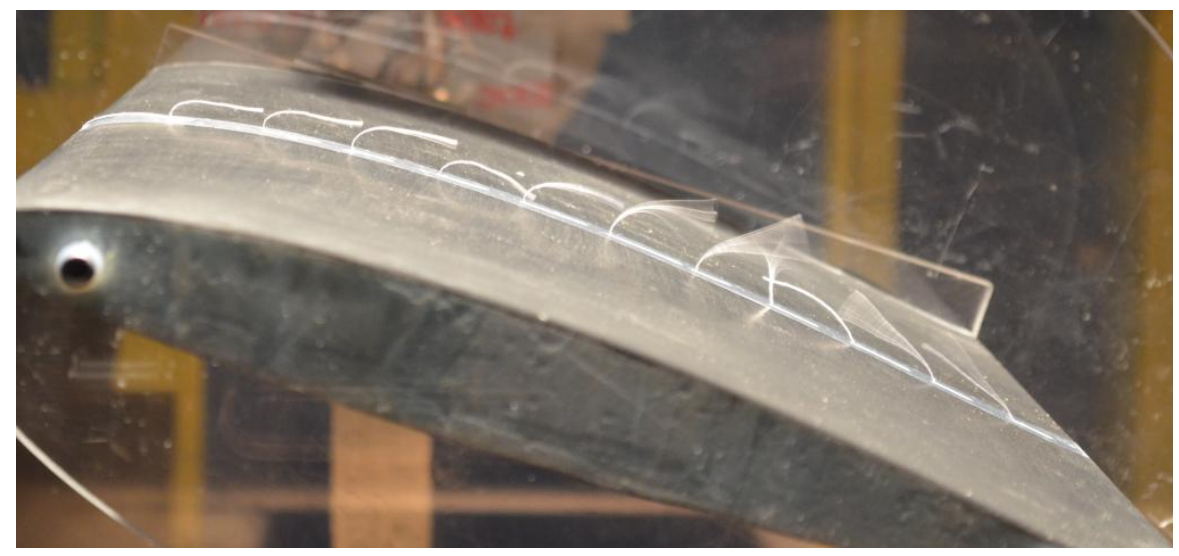

Figure 5-5 SD6060 undergoing reattachment at $18^{\circ} .\left(\operatorname{Re}_{\mathrm{c}}=150,000\right)$

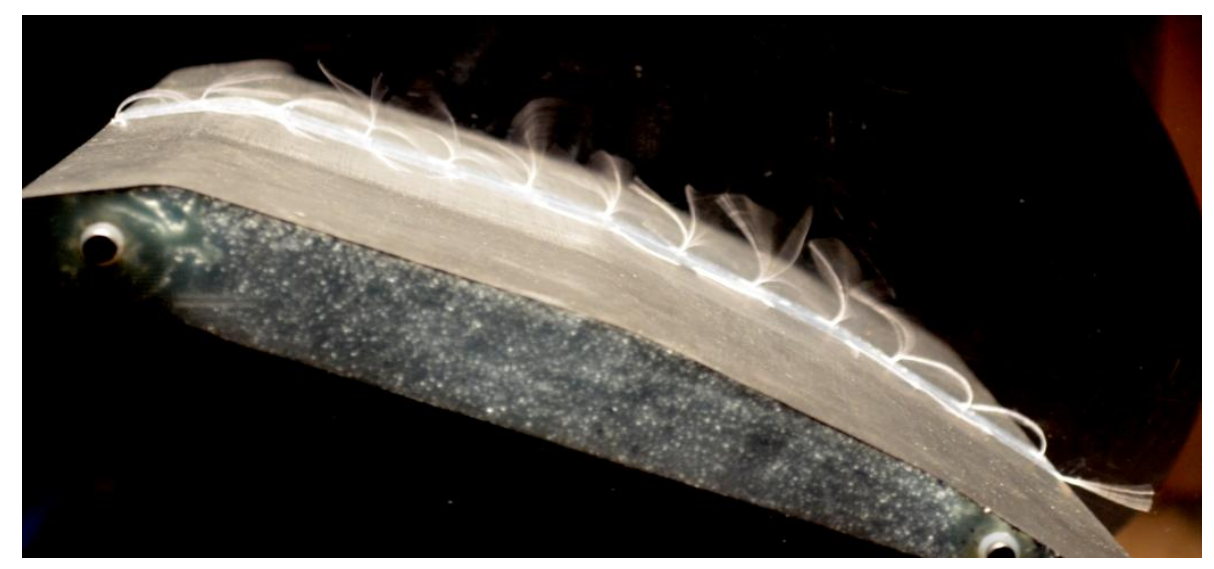

Figure 5-6 SF25158_20 experiencing full separation and recirculation at $18^{\circ} .\left(\operatorname{Re}_{c}=150,000\right)$

The experimental data for the SD6060 at $\operatorname{Re}_{\mathrm{c}}=150,000$ presented in Figure 4-3 is in disagreement with the the wind tunnel data provided by the tufts in Figure 5-5. The data collected by Fox indicates that $\alpha_{\text {stall }}$ occurs at approximately $14^{\circ}$, whereas the tufts in the figures above show that the SD6060 has not completely reached stall at $18^{\circ}$. It is possible that downwash is present in the WVU subsonic wind tunnel. If this is significant it could explain the discrepancy. However, this should be generally the same for each of the airfoils presented in this section. 


\subsection{Experimental Flow Visualization Summary}

This chapter of experimental flow visualization focused on comparing two airfoils: the SD6060 and the SF25158_20. The results can be summed-up a follows:

- Up to the point of stall, the SF25158_20 does not display a qualitatively significant difference from the SD6060 in regards to the location of its chord-wise separation location.

- Tufts do not indicate that the SF25158_20 is able to maintain attachment beyond that of the SD6060, either by use of laminar separation bubbles like that of the dragon fly wing nor through inducing early turbulent transition.

- Tufts show the periodic formation of a long-bubble on the trailing-edge of the SD6060.

- Although not outperforming the SD6060, the ability of the SF25158_20 to maintain similar attachment through the leading edge and mid-chord up to point of stall is of interest because the SD6060 has been contoured for inducing the preferred pressure gradients for maintaining attachment at low Reynolds.

- A mechanism for this is not immediately apparent with tufts. There is no apparent leading-edge circulation maintaining leading-edge attachment, and the possibility of turbulence generation in the boundary-layer is irresolvable without alternate methods. The possibility also exists that at low Reynolds the ShopFoil shape is generating the necessary pressure gradient to maintain attachment without the need for a turbulent trip or separation bubble.

- Despite the uncertainty as to the physical mechanism, the ability of the SF25158_20 to maintain attachment in addition to its inherent high lift capabilities may allow it to outperform the SD6060 experimentally. 


\section{Chapter 6 Comparing CFD and Experimental Flow Visualization}

The $\kappa \omega$-SST turbulence model has demonstrated its ability to accurately predict lift in low Reynolds flows. Here a qualitative comparison will be made of the air flow predicted by the twodimensional CFD runs and that which was observed in experiment through the use of tufts.

\subsection{Leading-edge separation bubble}

An interesting effect which was noticed among all the ShopFoils tested in CFD was the presence of a small separation bubble at the leading-edge at a Reynolds number of 120,000, which is shown in Figure 6-1. A similar separation bubble was also documented for the NACA 0009 using the same $\kappa \omega-\mathrm{SST}$ model and settings at low Reynolds [2]. This bubble formed numerically at all tested angles-of-attack presented in Figure 4-5 and Figure 4-6.

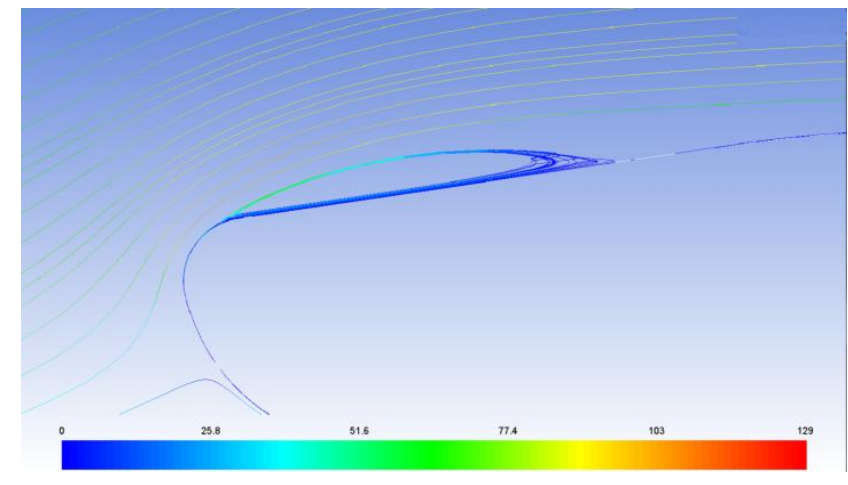

a) SF15126_10 at $6^{\circ}$

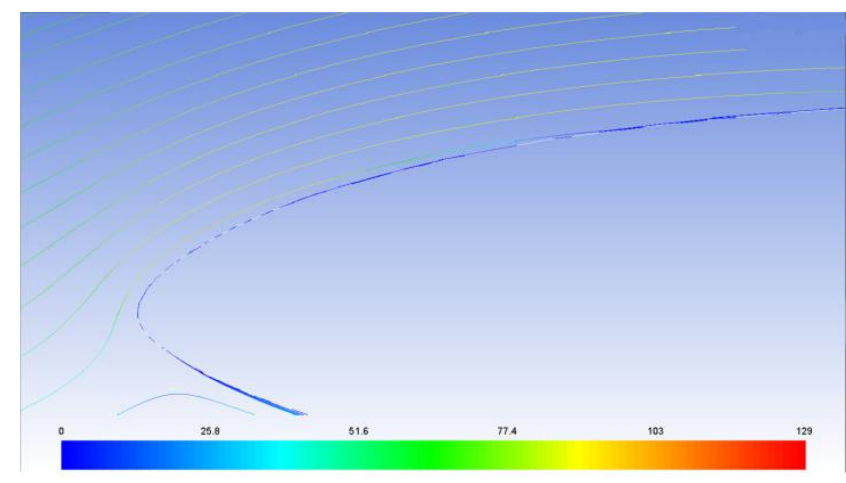

c) $\mathrm{SD6060}$ at $6^{\circ}$

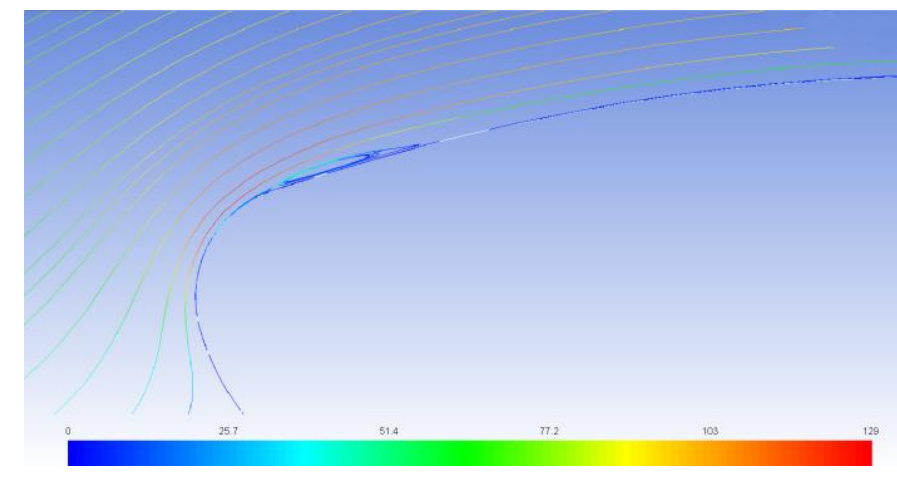

b) SF25158_20 at $6^{\circ}$

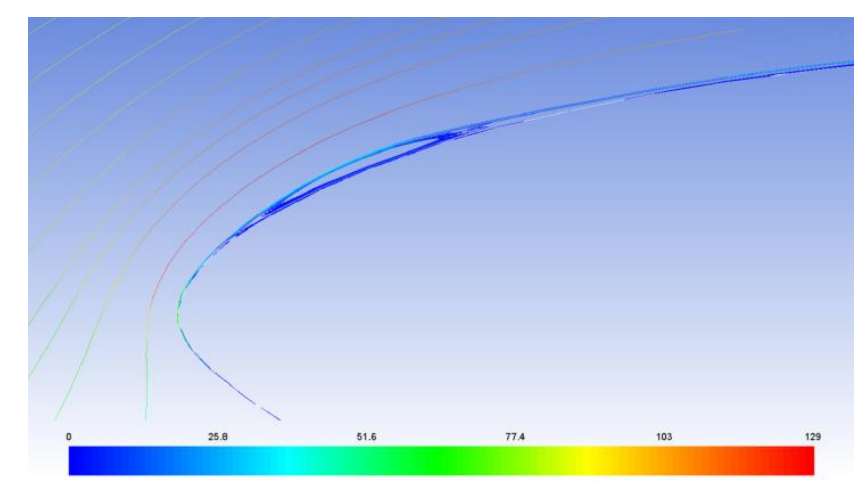

d) SD6060 at $10^{\circ}$

Figure 6-1 Separation bubble at leading-edge of an SF15126_10,SF25158_20 and SD6060.

$$
\left(\operatorname{Re}_{\mathrm{c}}=\mathbf{1 2 0 , 0 0 0 )}\right.
$$


The ShopFoils in Figure 6-1 a) and b) both demonstrate a leading-edge laminar separation bubble, here at $\alpha=6^{\circ}$, but are not limited to this angle. The flow around the SD6060 in c) does not form a bubble at $6^{\circ}$, however, one does form at $10^{\circ}$. It can be noted that the steeper leading-edge angle of $25^{\circ}$ on the SF25158_20 in b) forms a smaller bubble than the shallower angle of $15^{\circ}$ in c). This seems intuitive as the flow coming around the small radius leading-edge on the SF25158_20 requires a smaller turn to maintain attachment. The leading-edge bubble may play some role in effective shape as is the case with the dragonfly wing, if so, proper "tuning" of this leading-edge angle $\left(\beta_{1}\right)$ may yield beneficial results.

However, the formation of a short laminar bubble near the leading-edge has been well documented as discussed in Chapter 2. Thus, it is not exceptionally surprising to see this phenomenon develop numerically. Whether the bubbles present on the ShopFoils result from the same physical phenomenon as typical laminar short bubbles on other airfoils at low Reynolds number or are simply the result of geometry cannot be determined.

According to the flow visualization there seem to be cases for each airfoil at a $\operatorname{Re}_{\mathrm{c}}$ of 50,000 for which there is the possibility of a laminar bubble. At the leading edge of the SF20158_20 shown in Figure 6-2 there appears to be a change in the behavior of the tuft from $12^{\circ}$ to $14^{\circ}$. Once the angle of attack changes the second tuft acts as though chord-wise flow has halted. This could indicate the presence of a weak laminar short bubble on the leading edge. Images do reveal some movement of this tuft, however, it is minor. A similar phenomenon occurred for the SF25128_25 at the same Reynolds number.
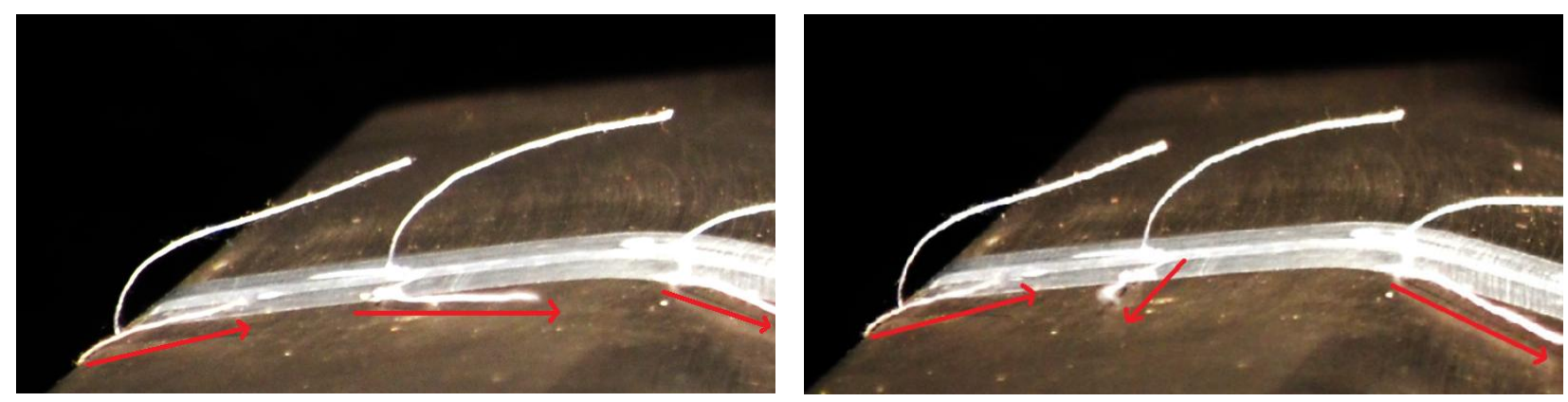

Figure 6-2 Leading edge comparison of SF20158_20 at $12^{\circ}($ left $)$ and $14^{\circ}$ (right). $\left(\operatorname{Re}_{\mathrm{c}}=50,000\right)$ 
As Reynolds numbers increased to 100,000 and 150,000 these effects seemed to disappear which is indicated by the tufts in Figure 6-3. The tufts on the SF25158_20 in this image are aligned with the free-stream and not displaying any signs of a circulating region the leading-edge. The photo in Figure 6-3 was taken at a Reynolds number of 150,000 and $\alpha=10^{\circ}$, although photos at 100,000 and 50,000 at all angles-of-attack corroborate this finding. The lack of visible evidence in this case does not, however, ruleout the possibility that such a region is not physical as it may be too small or too weak to entrain the tufts. This evidence does seem to suggest that any global or leading-edge flow influence by a laminar bubble is minimal, if not negligible, at these larger Reynolds numbers.

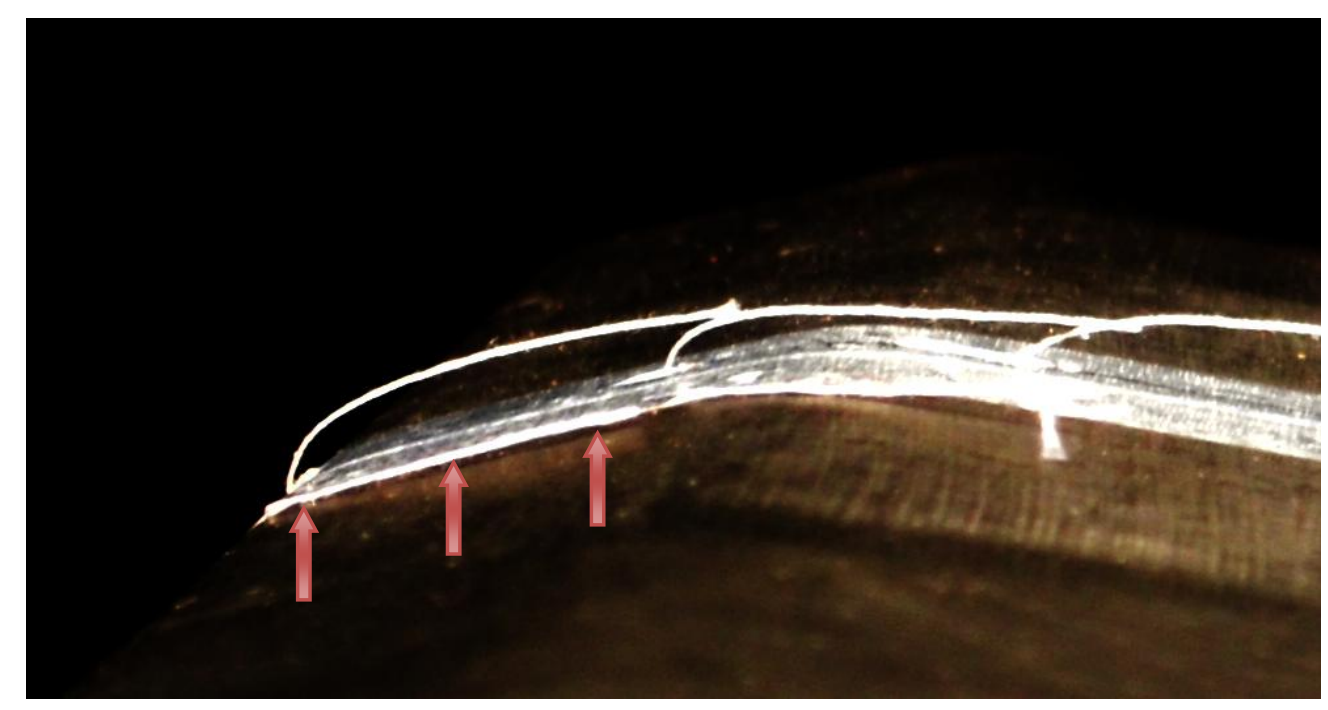

Figure 6-3 Tufts at the leading-edge of an $S F 25158 \_20$ aligned with free-stream $\left(\operatorname{Re}_{c}=150,000\right)$.

\subsection{Separation: onset and progression}

Another important factor is how well the $\kappa \omega$-SST model is able to model the progression and manner of separation as this will directly affect the reliability of lift and drag results. Experimental data indicates the CFD model represents the flow characteristics relatively accurately as far as the separation point is concerned up to about $10^{\circ}$. Experimental flow visualization using tufts for the SF25158_20 at $10^{\circ}$ in Figure 6-4 indicates that the flow remains attached until the location of the $\beta_{3}$, or approximately the $50 \%$ chord location. 


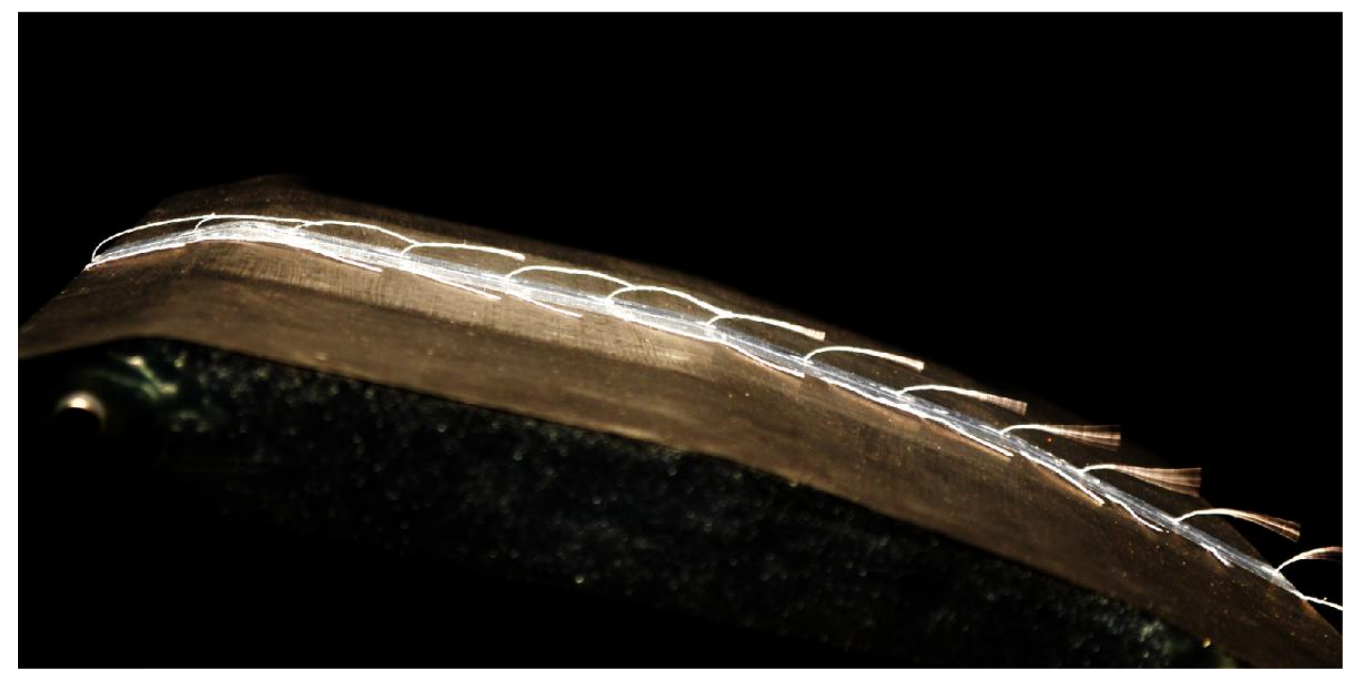

Figure 6-4 Tufts indicating trailing edge separation on a SF25158_20 at $10^{\circ}\left(\operatorname{Re}_{\mathrm{c}}=150,000\right)$.

A zoom-in of the tufts at the trailing edge of the SF25158_20 is provided in Figure 6-5. The range of motion (envelope) of the tufts is highlighted with the red brackets and does not appear to involve any periodic reversed flow, as would be the case in laminar shedding, but is mostly aligned with the freestream.

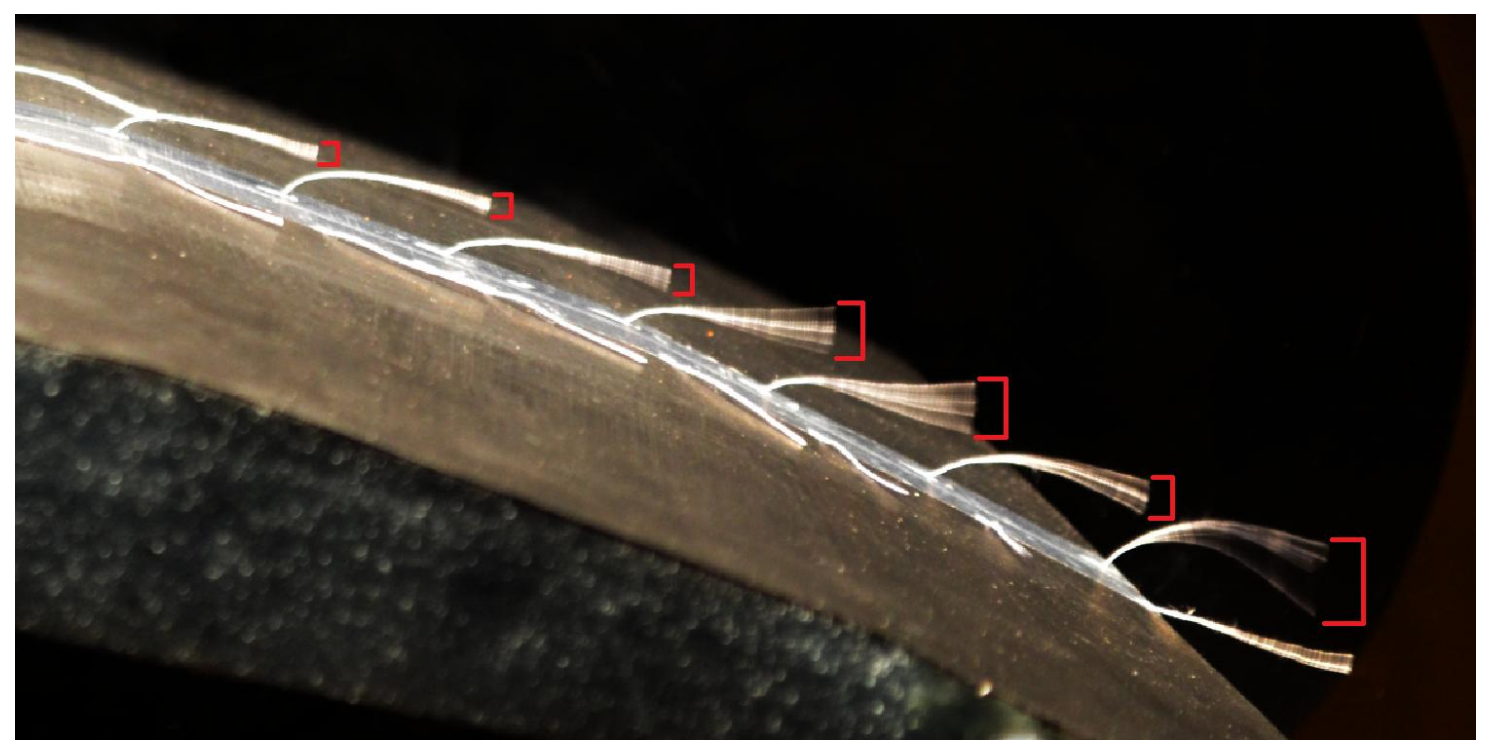

Figure 6-5 Zoom-in of tufts on the SF25158_20 at $10^{\circ}\left(\operatorname{Re}_{\mathrm{c}}=150,000\right)$. 
The two-dimensional velocity vector plots in Figure 6-6 shows very similar results; aside from the very small separation region from Figure 6-1, the flow remains attached through the leading edge up to the mid-chord with separation starting at $\beta_{3}$ (approximately $60 \%$ chord).

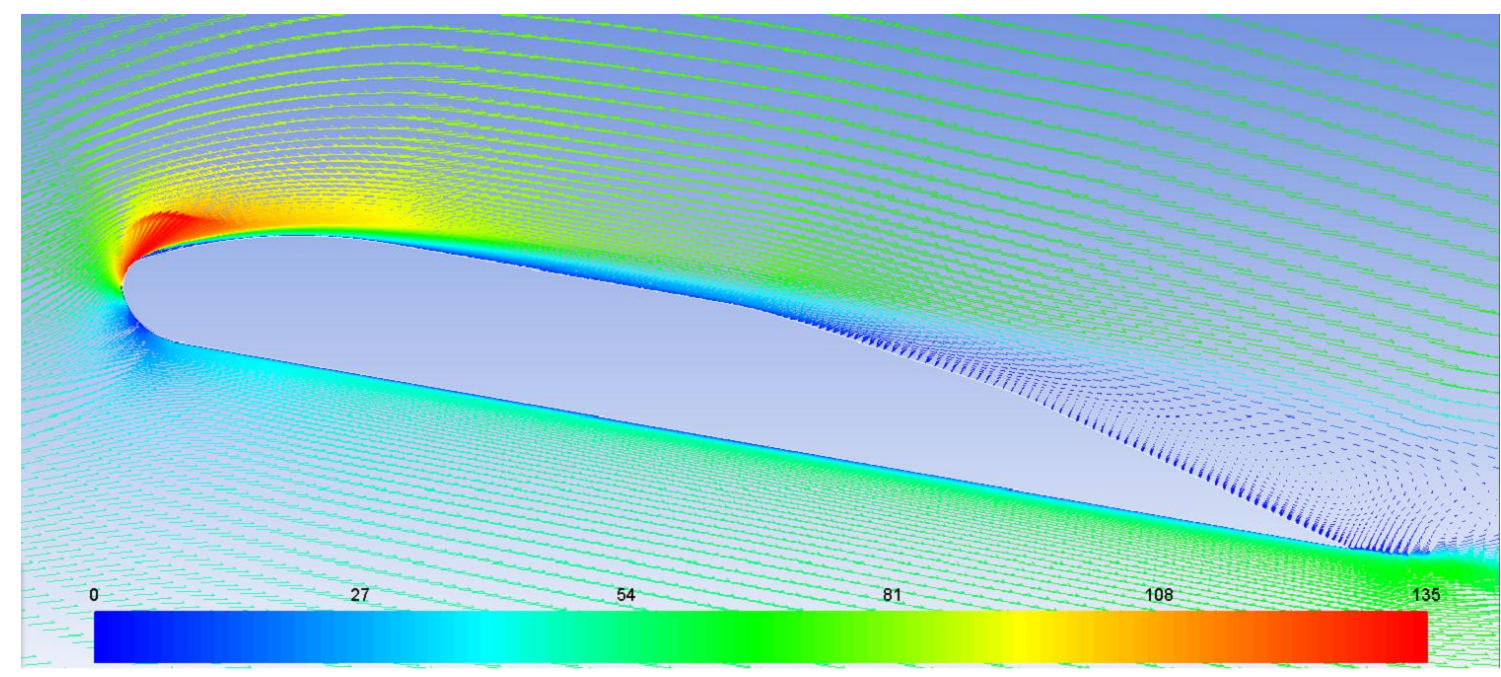

Figure 6-6 Two-dimensional velocity vector plots for $S F 25158 \_20$ at $10^{\circ}\left(\operatorname{Re}_{c}=120,000\right)$.

Considering the nature of the separation predicted by the $\kappa \omega-S S T$ model in Figure 6-7, it can be seen that the separation results in the periodic shedding of vortex structures with a size on the order of the airfoil thickness. Contrasting this with the experimental results in Figure 6-5, which do not indicate the presence of any vortex structures of such scale being shed from the trailing edge, there appears to be consistency regarding the point of separation but not in form. The experimental evidence seems to suggest turbulent shedding. 


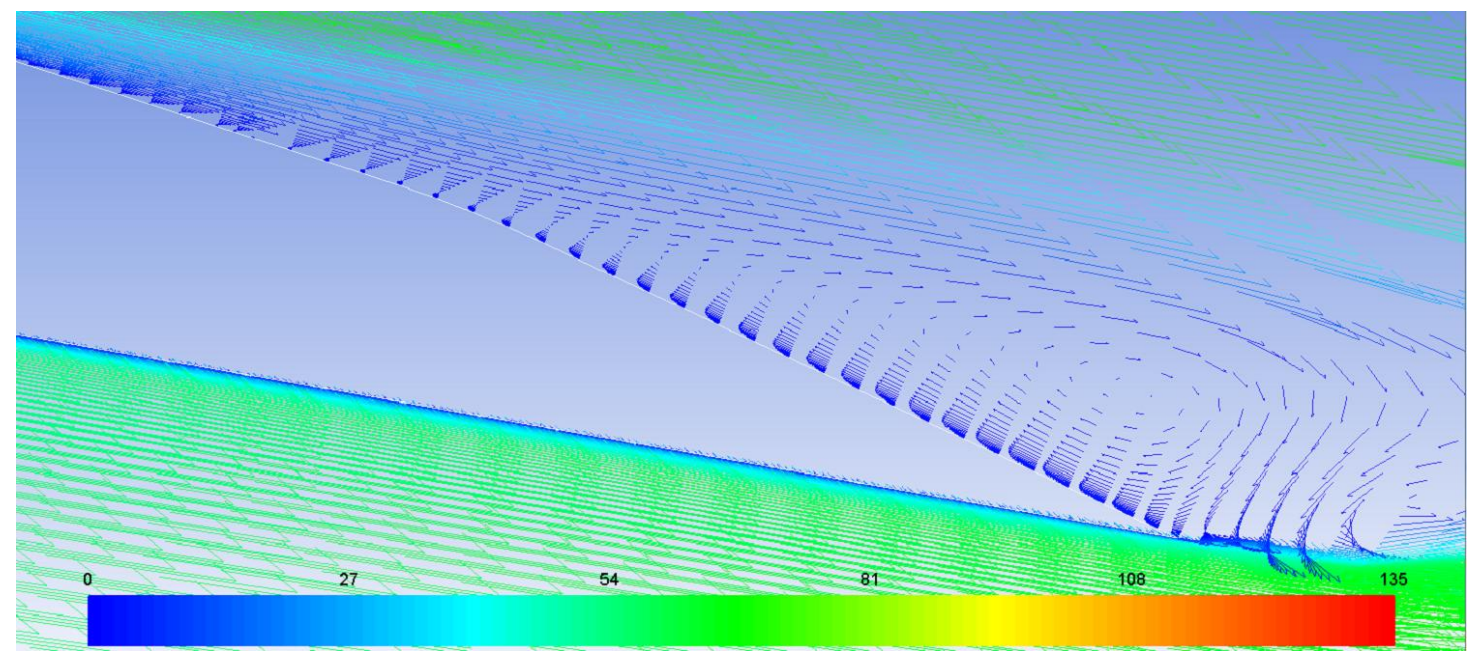

Figure 6-7 Two-dimensional velocity vector plots of the trailing-edge of the $S F 25158 \_20$ at $10^{\circ}\left(\operatorname{Re}_{c}=\right.$ $120,000)$.

Beyond this angle of attack there is little correlation between the $\kappa \omega$-SST model and experiment. To demonstrate this, refer to Figure 6-8 and notice that the point of separation occurs just after the leading-edge at the $\beta_{1}$ angle. Additionally, there is an attached reverse flow along the entire upper surface and large-scale laminar shedding up to the point of separation. However, in Figure 6-9 the separation point is nearly indistinguishable from that at $10^{\circ}$ in Figure 6-4, as flow attachment is maintained from the leading-edge to the $\beta_{2}$. There is no evidence of an attached recirculation zone nor does there appear to be large-scale laminar shedding.

However, as noted in Chapter 5, the WVU subsonic tunnel has been noted to occasionally have a downwash component, recorded as high as $-3^{\circ}$. Additionally, the wind tunnel has a real turbulence intensity component, which has been measured in by previous experimenters to be around $2 \%$, and could potentially affect the separation angle of attack. The CFD model on the other hand, has a numerical turbulence intensity of $1 \%$. Whether or not this is a factor in the discrepancy has been left to future research. 


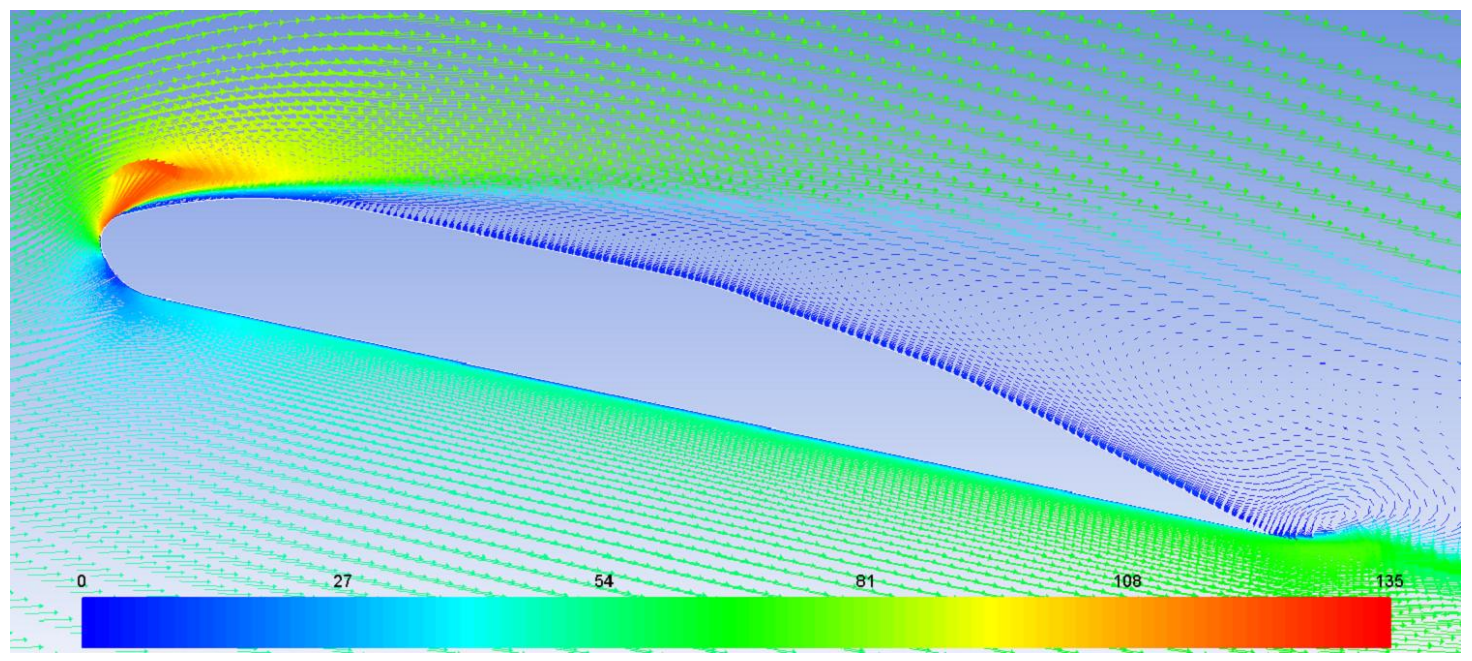

Figure 6-8 Two-dimensional velocity vector plot of the $S F 25158 \_20$ at $12^{\circ}\left(\operatorname{Re}_{\mathrm{c}}=120,000\right)$.

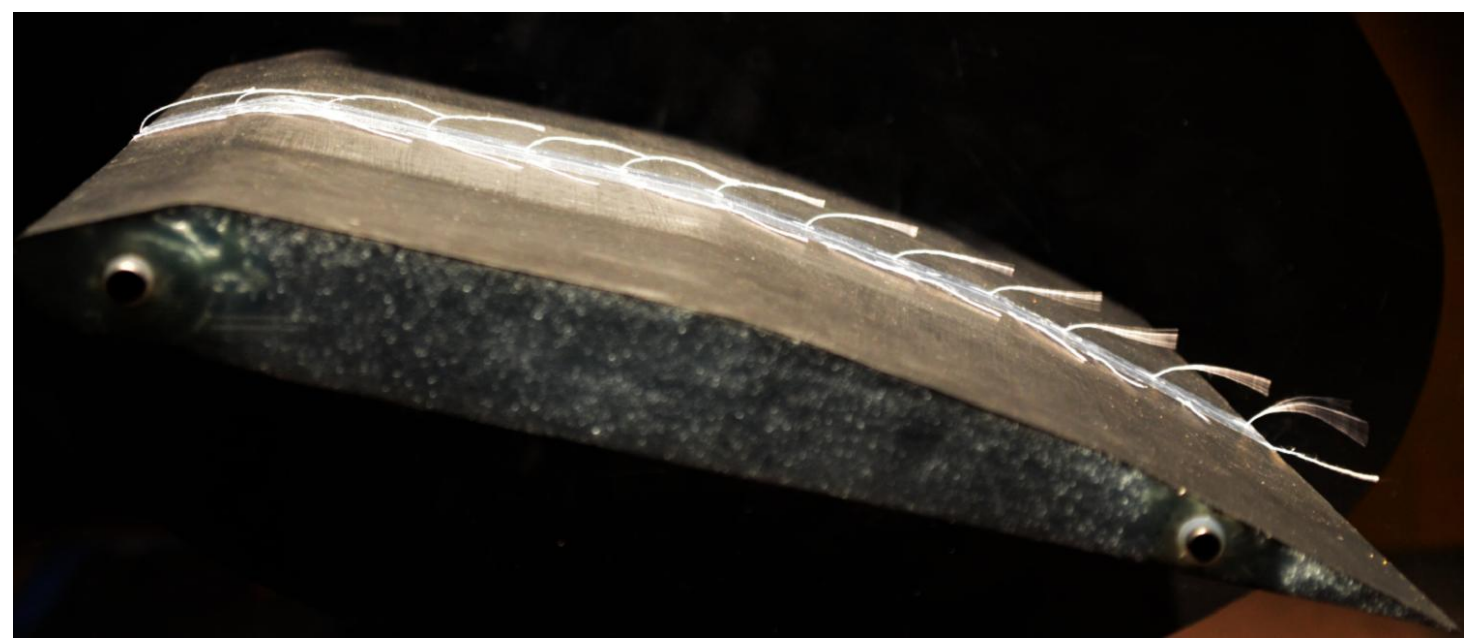

Figure 6-9 Tufts on SF25158_20 at $12^{\circ}\left(\operatorname{Re}_{\mathrm{c}}=150,000\right)$.

These differences and similarities are not isolated to the ShopFoil sections but similar behavior also occurs with the SD6060 as will be shown in Figure 6-10 through Figure 6-13. Proceeding in similar fashion as above for the SF25158_20, the CFD and experimental data will be compared at only $12^{\circ}$ to illustrate their disagreement and to avoid redundancy. Flow visualization for the SD6060 at $12^{\circ}$ is provided in Figure 6-10 and shows that attachment is maintained for at least 50\% of the chord length as the majority of the shedding occurs toward the trailing edge. The magnitude and extent of the shedding is more clearly evident in the zoom-in photo of Figure 6-11 and again highlighted in red showing the range of the motion. As with the SF25158_20 the motion does not seem to indicate any periodic shedding of large laminar vortices but rather small-scale motion. 


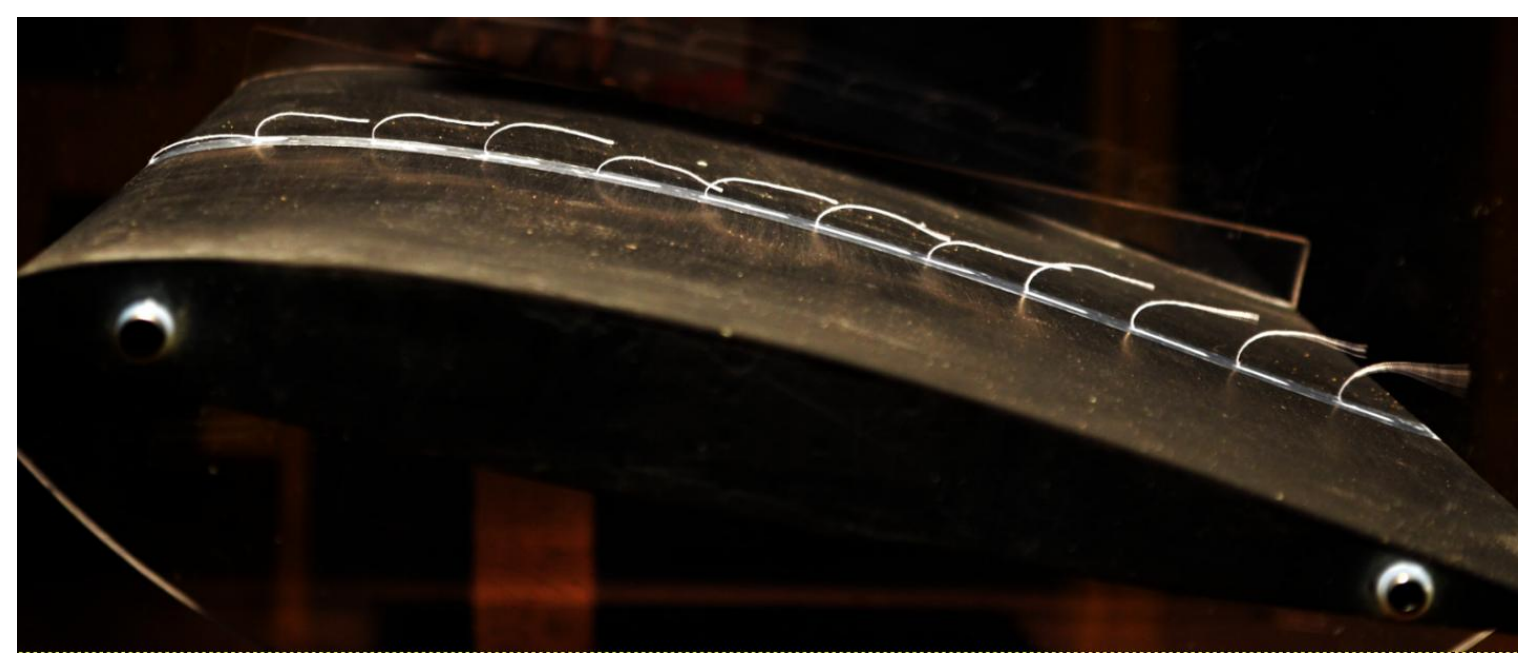

Figure 6-10 Tufts on SD6060 at $12^{\circ}\left(\operatorname{Re}_{\mathrm{c}}=150,000\right)$.

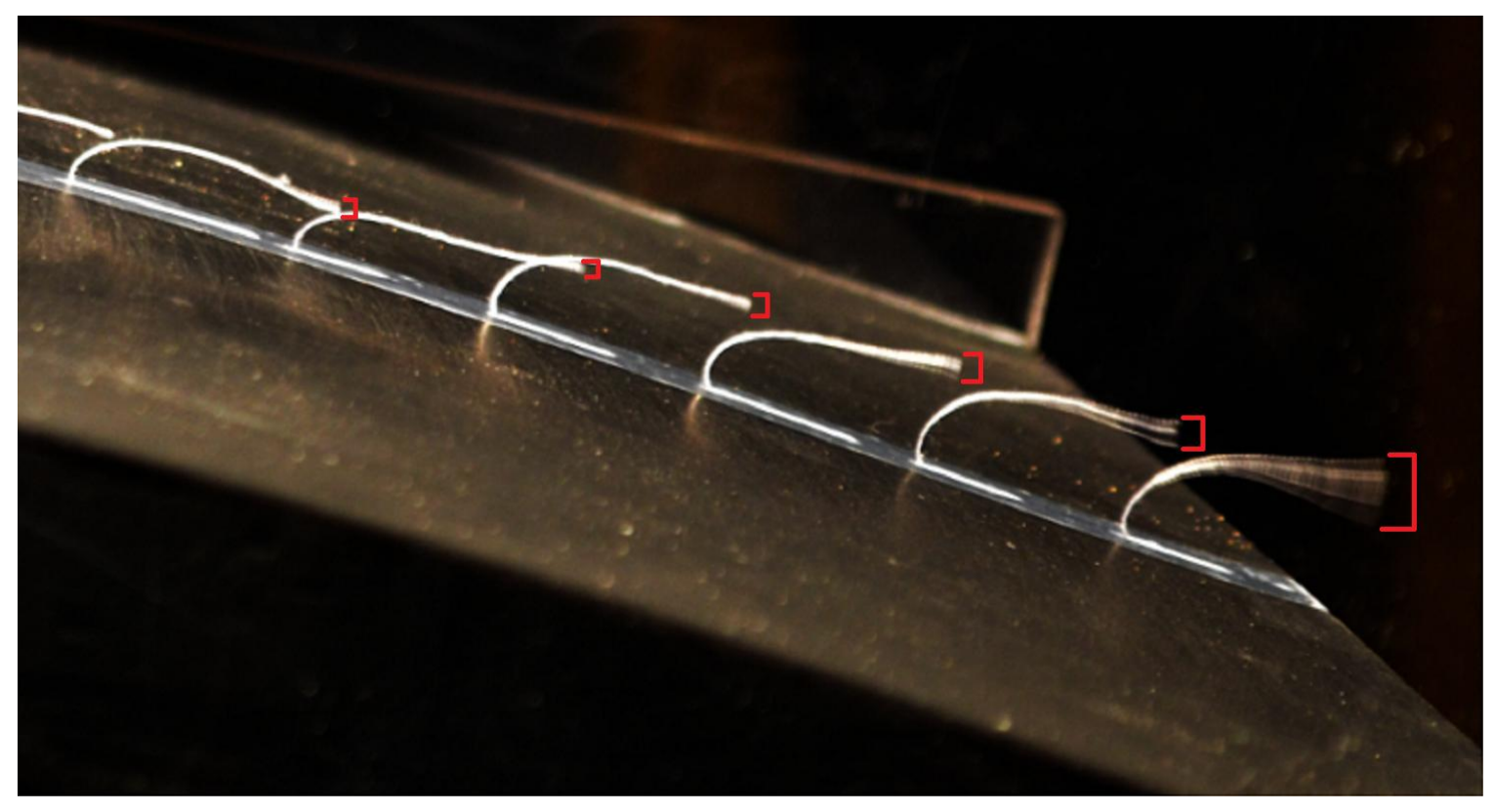

Figure 6-11 Zoom-in of tufts on trailing-edge of $\operatorname{SD6060}$ at $12^{\circ}\left(\operatorname{Re}_{c}=150,000\right)$.

Comparing this with the numerical results in Figure 6-12 it can be seen that the $\kappa \omega$-SST model indicates that separation has progressed passed the 50\% chord location, there is a backflow region at the airfoil surface proceeding from the trailing-edge to the point of separation and large-scale vortex shedding shown in Figure 6-13. 


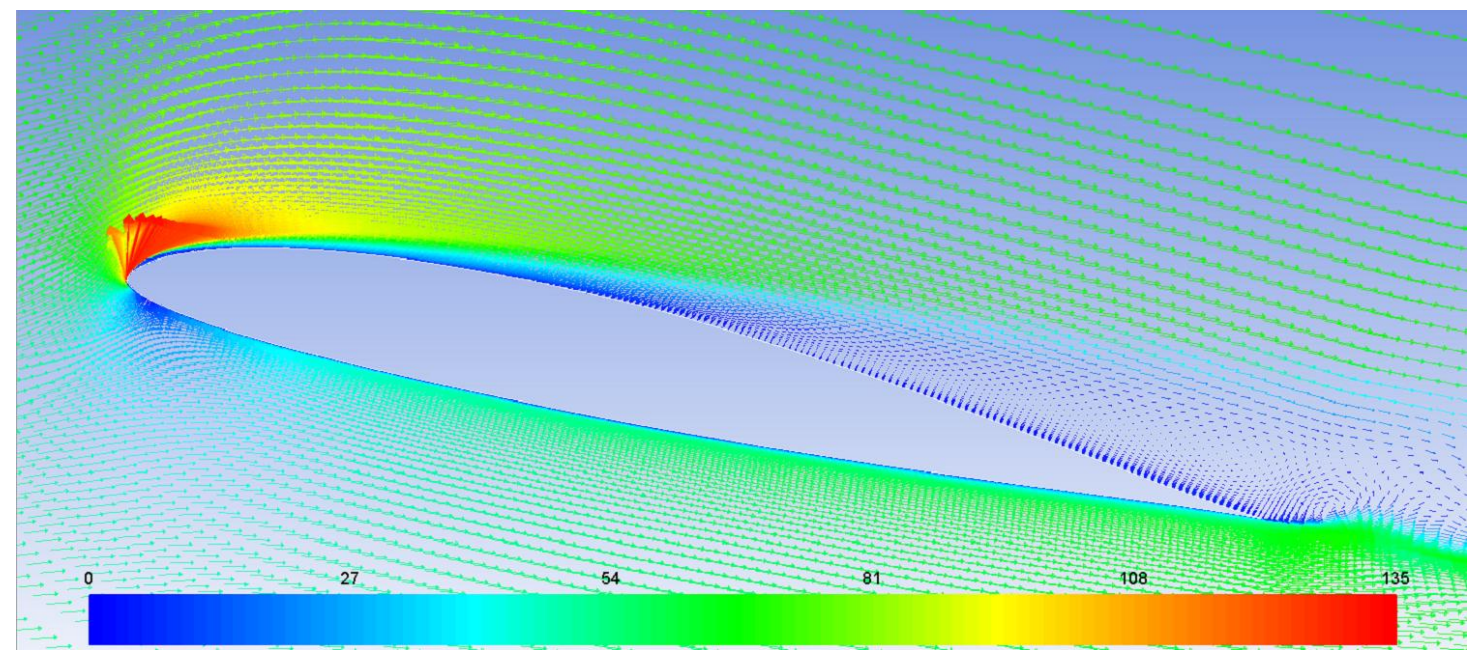

Figure 6-12 Two-dimensional velocity vectors of the $\operatorname{SD6060}$ at $12^{\circ}\left(\operatorname{Re}_{\mathrm{c}}=120,000\right)$.

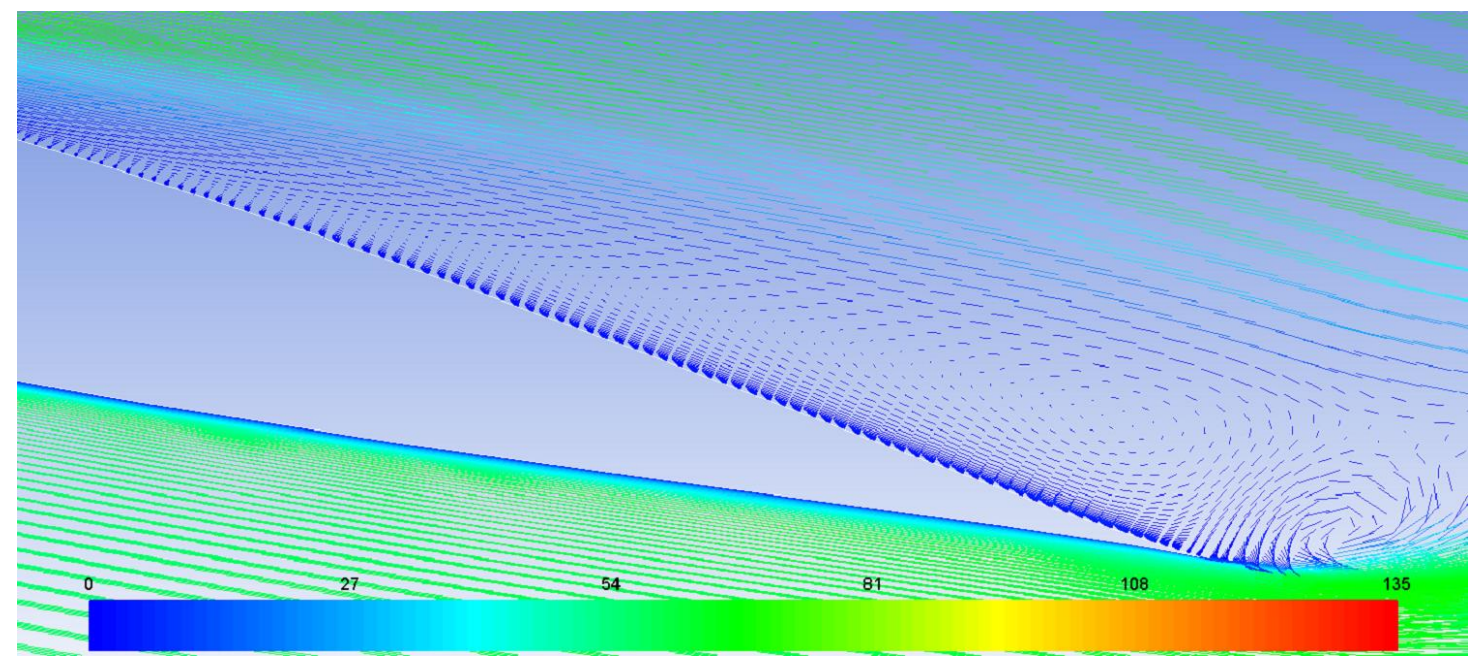

Figure 6-13 Zoom-in of velocity vectors at the trailing-edge of the $\mathrm{SD6060}$ at $12^{\circ}\left(\operatorname{Re}_{\mathrm{c}}=120,000\right)$.

\subsection{Summary}

- There appears to be some experimental evidence for the laminar separation bubble predicted by the $\kappa \omega$-SST model and literature at the leading-edge of the ShopFoils at a Re of 50,000, but this effect does not extend to the higher Re of 100,000 and 150,000. It also does not appear to induce any beneficial flow qualities.

- The $\kappa \omega$-SST model is qualitatively similar to the flow visualization data for both the SD6060 and SF25158_20 in regards to attachment up to approximately $10^{\circ}$.

- At around $12^{\circ}$ the CFD turbulence model appears to over-predict stall progression for both types of airfoils.

- The experimental data suggests turbulent shedding for both foils whereas the turbulence model predicts shedding in the form of large-scale eddies. 


\section{Chapter 7 Resolution of ShopFoil Performance}

The aim of this chapter is to provide a potential explanation for the predicted performance of the ShopFoil, both in the CFD results of Figure 4-8 and the experimental results. Experimental data for the ShopFoil sections does not suggest that a mechanism such as laminar separation bubbles nor structuraleffective turbulent trips are acting to maintain attachment beyond that of the SD6060; thereby causing better performance.

Recall, however, that there was a discrepancy in the two-dimensional and three-dimensional numerical lift-to-drag plots. This seemed to hint that the performance difference was related to threedimensional effects. As was noted, experimental data also confirmed the similar performance of these airfoils. While the comparisons of Chapter 6 indicated some differences between the experimental and numerical results, it was also concluded that attachment was predicted relatively accurately up to $10^{\circ}$ and that the divergence from the experimental results was consistent across both the ShopFoil and SD6060. Therefore, the numerical results along with finite wing theory will be consulted to further understand this phenomenon.

There are a few fundamental concepts regarding finite wing theory which are useful for understanding the reason for these results and it may be beneficial to provide a quick review before proceeding; so, this chapter will be presented as part literature review, part results, and part conclusions.

\subsection{Finite Wing Theory}

The fundamental relationship between the lift generated at any location on a wing and the local circulation is provided by the Kutta-Joukowski Theorem:

$$
\boldsymbol{l}(\boldsymbol{y})=\boldsymbol{\rho}_{\infty} \boldsymbol{U}_{\infty} \Gamma(\boldsymbol{y})
$$

From Lanchester-Prandtl's Lifting-line theory the span-wise lift distribution is represented by a system of bound vortex filaments; "at any span-wise location y, the sum of the strengths of the vortex filaments in the bundle at that station is $\Gamma(\mathrm{y})$. When the lift changes at some span-wise location [i.e., $\Delta l(y)$ ], the total strength of the bound vortex system changes proportionally [i.e., $\Delta \Gamma(y)][31][32] . "$ Additionally, according to a particular vortex theorem of Helmholtz, a vortex cannot end in the fluid. It must form a closed path, end at a boundary, or go to infinity. 
For a finite wing there exists neither a boundary at which the circulation may end nor a surface about which to maintain circulation in the span-wise direction out to infinity, thus a continuous closedloop is formed back to the starting location through the stream-wise shedding of the bound vortex. Any incremental change in the strength of the bound vortex system implies the shedding of a vortex equal in strength to $\Delta \Gamma(y)$ in the stream-wise direction where,

$$
\Delta \Gamma=\frac{d \Gamma}{d y} \Delta y
$$

For a finite wing represented by a lifting-line, the span-wise loss in circulation results in the shedding of two stream-wise, counter-rotating vortices at each wing-tip. These vortices induce a downward velocity $w$ component aft of the wing called downwash which adds to the free-stream velocity vector $V_{\infty}$. The result is an altered flow direction by an angle $\alpha_{i}$, the induced angle. This effect is illustrated in Figure 7-1. Downwash affects the angle of attack which is seen by each section of the wing, progressively reducing the effective angle of attack out to the wing tip. The effective angle of attack is given by

$$
\alpha_{e f f}=\alpha-\alpha_{i}
$$
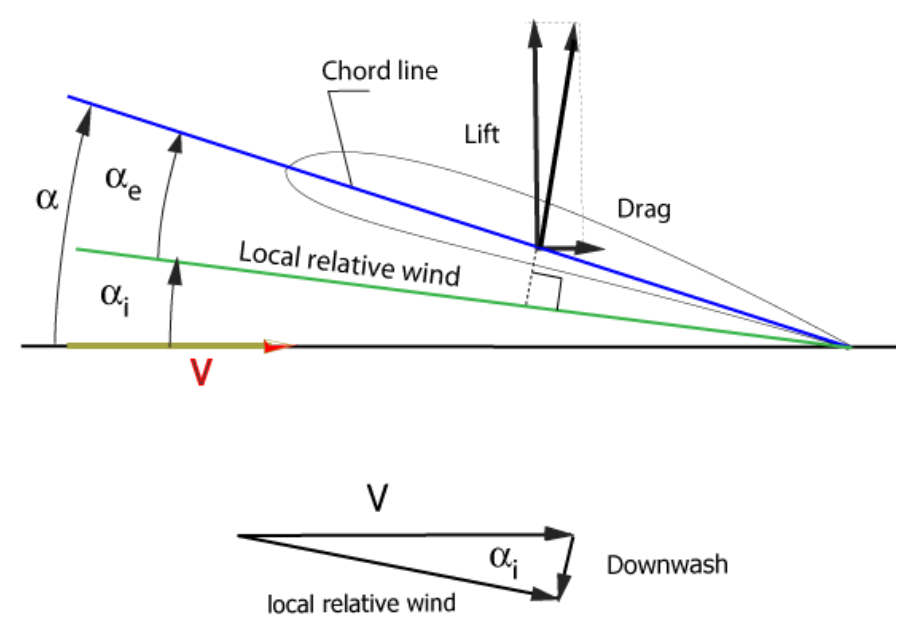

Figure 7-1 Effect of downwash on angle of attack. 
The rate of span-wise shedding and thus the downwash profile depends on the shape of the wing planform. Consider a rectangular wing: ideally, such a planform would see no loss in local circulation until reaching the tip where lift abruptly drops to zero and result in no loss of lift effectiveness across the span. Of course, this does not occur in reality as there is an interrelation between the effects of downwash and span-wise lift distribution; downwash reduces the lift toward the tip. However, the vortex shedding for a rectangular planform is still concentrated near the tips and thus the downwash profile is also greatest at the wing tips, as is shown in Figure 7-2 (c).

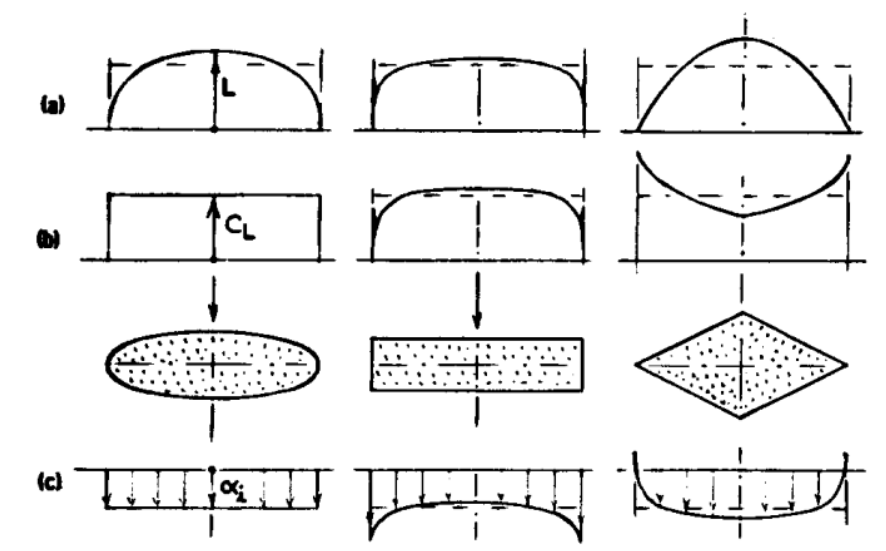

Figure 7-2 Downwash profiles for assorted wing planforms [33].

This tendency of rectangular wings to produce the greatest downwash toward the end of the wing is directly related to stall progression of these planforms, which begins at the root and moves toward the tips. For a rectangular wing of a given cross-section at angle of attack the center of the wing is the only portion which is experiencing the geometrical or true angle of attack and thus is the closest at any time to reaching the section $\mathrm{C}_{\operatorname{lmax}}$. Proceeding down the span toward the tip, each section along the way is progressively seeing the smaller effective angle of attack and necessarily lags the center section in reaching $\mathrm{C}_{\operatorname{lmax}}$; with the largest reduction in effective attack angle occurring near the tip. The stall progression of a rectangular wing can be seen in the photo of the surface oil flow in Figure 7-3. 


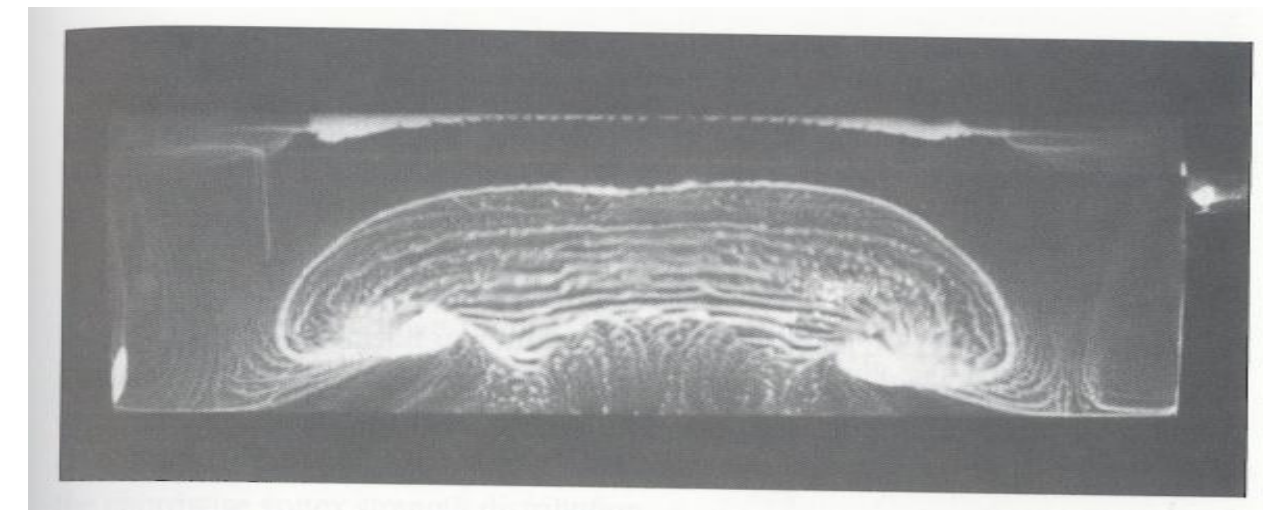

Figure 7-3 Oil flow of stall progression for a finite, rectangular Clark Y-14 planform of $A R=3.5, \alpha=22.8^{\circ}$,

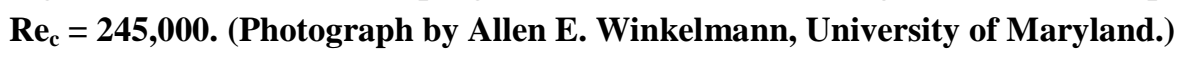

\subsection{CFD Span-wise downwash effects}

How does this relate to the performance of the ShopFoil on the HP configuration? Recall first that the vehicle in question has a rectangular planform; we should see a reduction in the effective angle of attack and smaller regions of separation associated with them concentrated near the tips of the wing in accordance with the downwash profiles in Figure 7-2. The sections closer inboard should see larger, more advanced stall regions associated with the larger geometric attack-angle.

By plotting velocity contours at sections along the span of the wing this effect should be visible. Contour plots are provided in Figure 7-4 for the SD6060 and SF25158_20 at a geometric angle of attack of $11.6^{\circ}$. The first pair of contour plots were taken at the root. From a qualitative standpoint they show smaller regions of separation and it is possible the body is influencing the flow here. If the remaining pairs of images are compared, as the wing tip is approached the progressive reduction in the magnitude of the separation region (dark blue) of both planforms becomes apparent. 

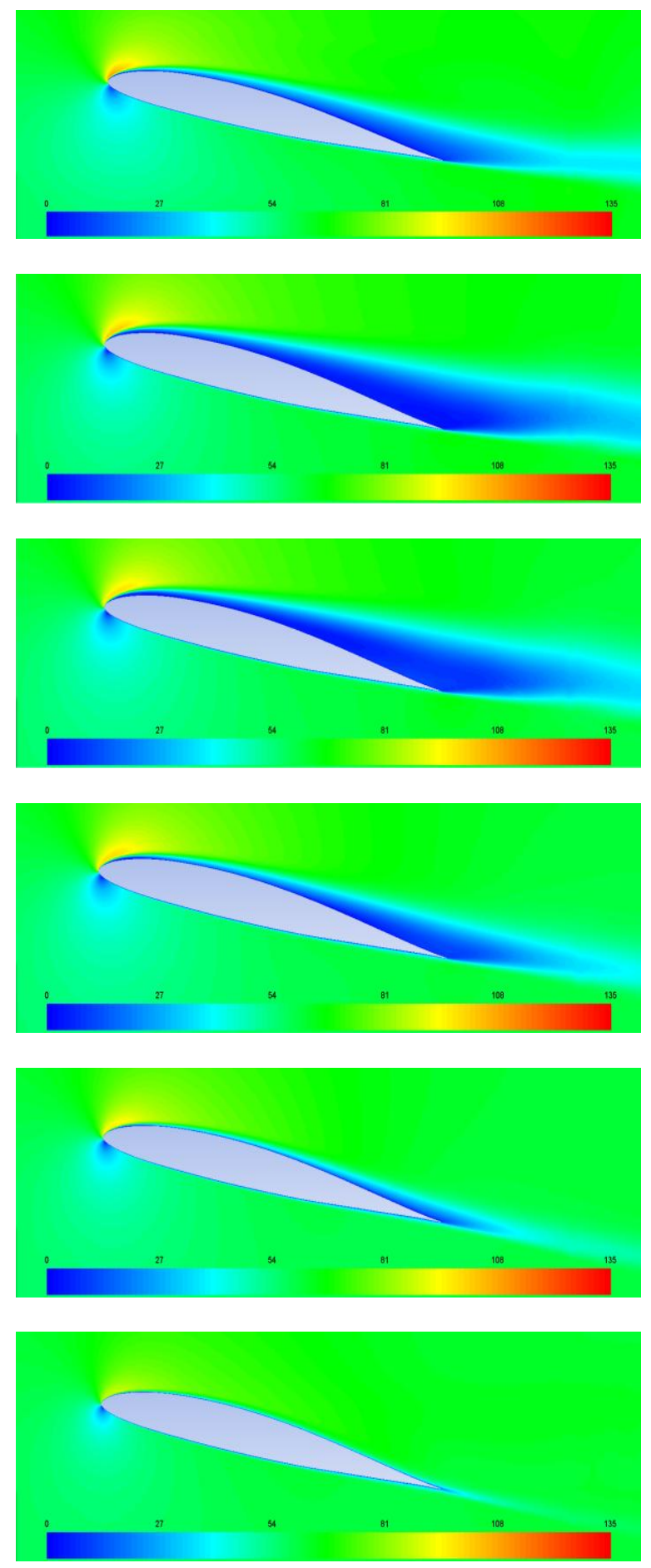

Figure 7-4 Velocity magnitude contour plots of the SD6060 (left) and SF25158_20 (right) along the span for $\alpha$ $=11.6^{\circ}$ showing reduced separation near the tip. These plots begin with the inboard location (top) and proceed to the tip (bottom). [Note: a visual comparison of the wake profiles of these airfoils may be misleading due to image scaling.]
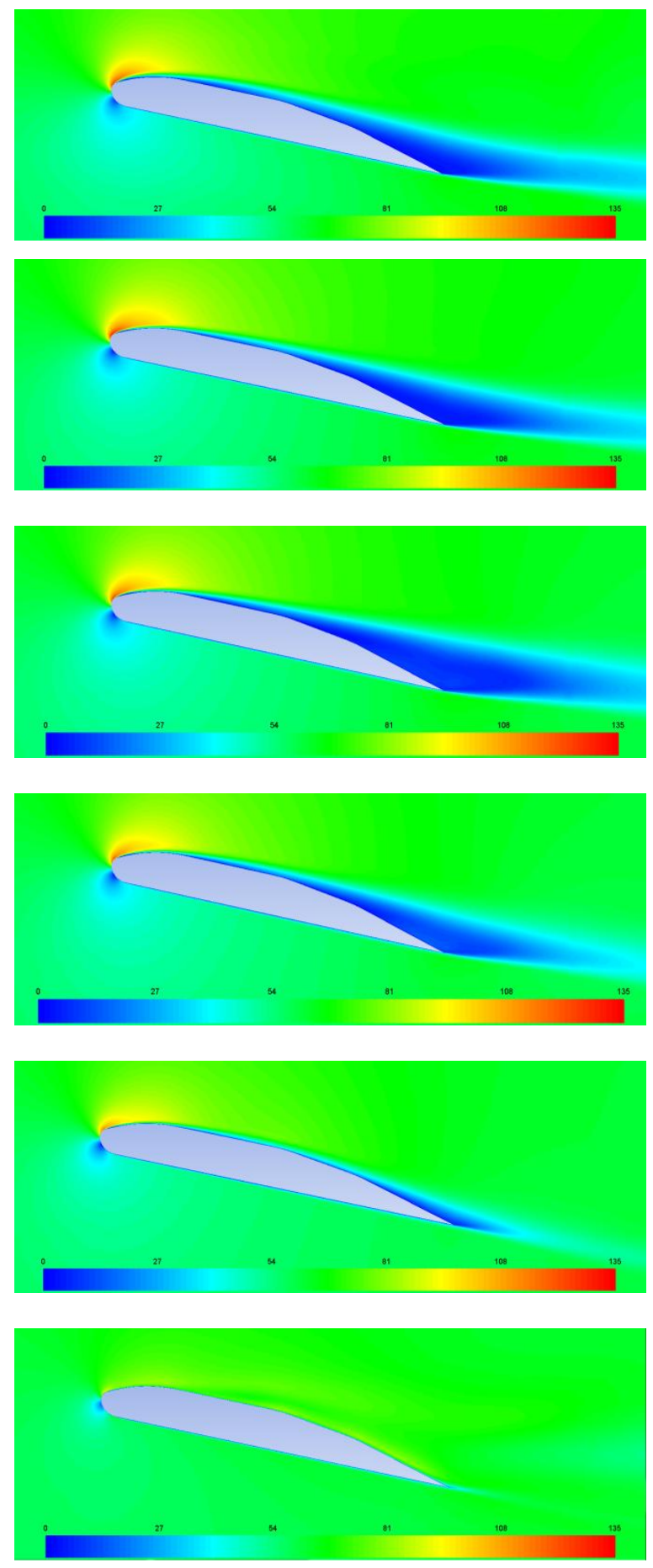
Additionally, further clarification of the spanwise separation behavior of the SF25158_20 is provided in Figure 7-5 which shows the steady reduction in the separated air flow as the tip is approached. This series of plots is complementary to the well-established effects of downwash and the stall progression flow visualization image in Figure 7-3, where the flow near the center of the wing is separated and the flow near the tips remains attached. Approximately one-third of the half-span appears to be under most of the downwash effects.

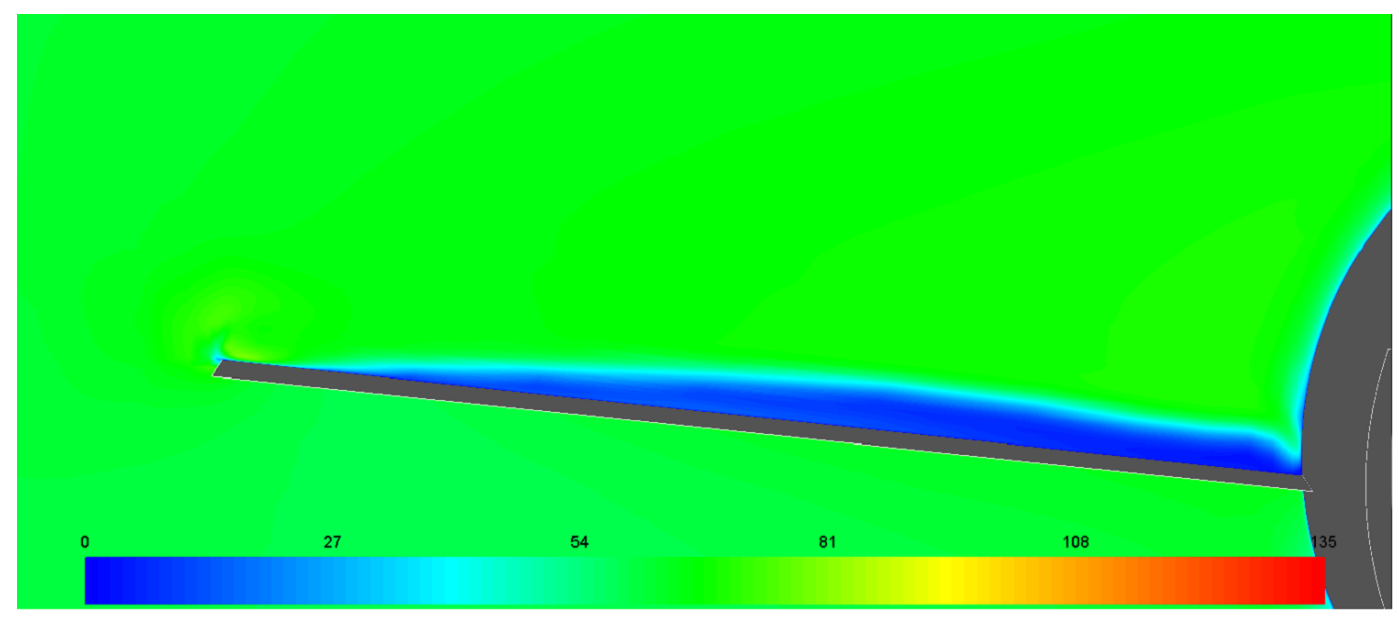

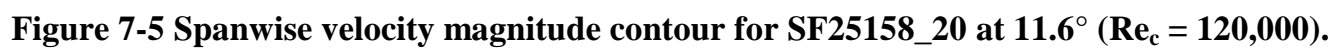

It is now possible to present a potential reason the ShopFoil planform achieves such similar performance to the SD6060 in three-dimensional numerical tests and wind-tunnel tests discussed in Chapter 4. Recall that the two-dimensional lift coefficient plots in Figure 4-7 indicate that the ShopFoil sections dominate in lift generation at smaller angles of attack and that some sections, such as the SF25158_20, produce higher lift than the SD6060 up to about $12^{\circ}$. As was demonstrated in Figure 7-4, spanning the wing from the root to the tip, the cross-sections progressively see a reduction in the effective angle of attack. This places the wing near the tip in the region of the lift-curve slope and the lift-to-drag curve where the ShopFoils are more effective; thus gaining a performance advantage over the SD6060. An example of this effect is shown in Figure 7-6. The blue line represents the geometric angle of attack; the red line represents the effective angle of attack in the vicinity of the tip, while the transparent red section marks the transition across the span. 


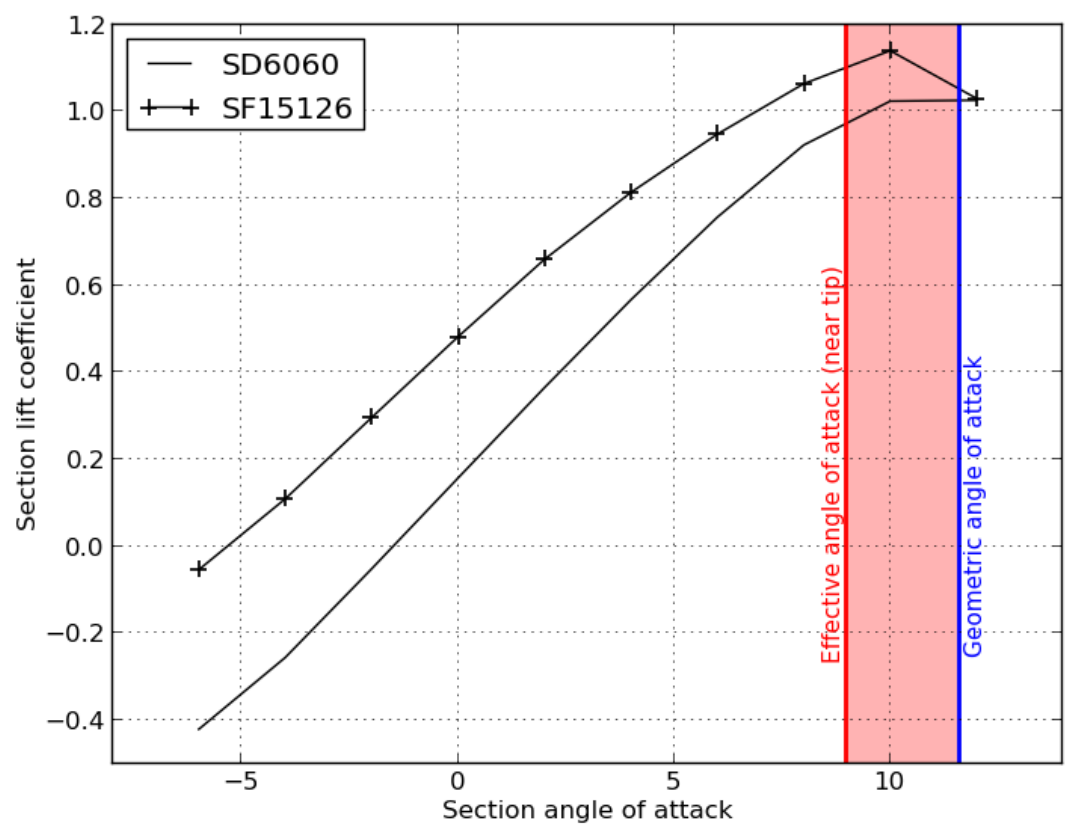

Figure 7-6 Effect of downwash on spanwise performance.

\subsection{Tip Vortex Review}

There may be more involved with the three-dimensional ShopFoil performance than simply the angle of attack reduction from downwash; the effective angle result stems directly from the simplified lifting-line theory and does not account for the real, three-dimensional effects of the "tip vortices" on the lift and induced drag [33]. In the actual situation of a finite wing, "tip vortices" result as soon as a pressure gradient is established between the upper and lower surface of the wing. Generally, because this pressure gradient occurs almost immediately, the vortices begin forming toward the leading-edge as opposed to the trailing edge. "The flow component from the lower side combines with the stream along the upper side of the wing tip, forming a tip vortex [33]." This process is illustrated in Figure 7-7.

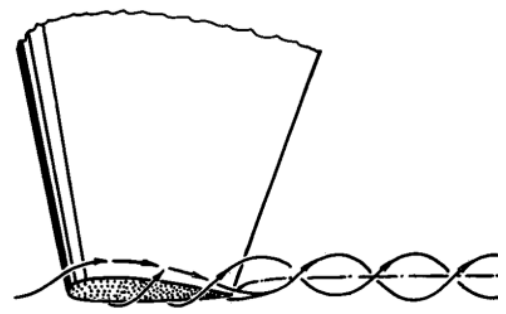

Figure 7-7 Flow pattern of tip vortex from Hoerner [34]. 
During formation, the "tip vortex" wraps around the lateral edge of the wing. The extent to which the vortex establishes itself along the upper surface is dependent on the contouring of the tip and may influence the effective span. For examples of the vortex core location for varying tip shapes refer to Figure 7-8.

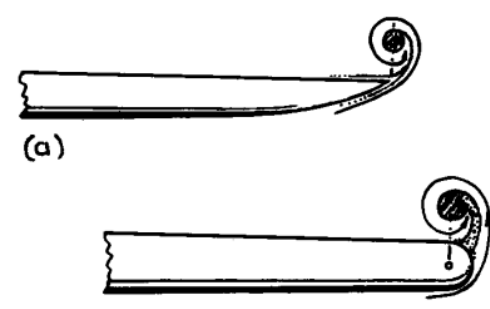

(b)

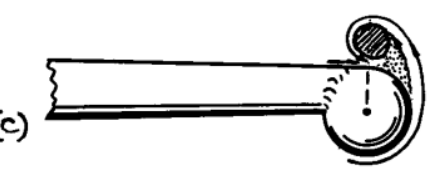

Figure 7-8 Vortex core locations for various tip contours from Hoerner [34].

The presence of a "tip vortex" along the upper surface of the wing is not unusual, as can be seen from a streamline plot from the numerical simulations of the wing-body configuration in Figure 7-9. The vortex can be seen beginning near the leading edge and sitting over the trailing edge. As mentioned previously, the 'tip vortex' is generally understood to reduce the effective span of the wing; an unfavorable result. However, vortices are commonly used to produce favorable results on wings.

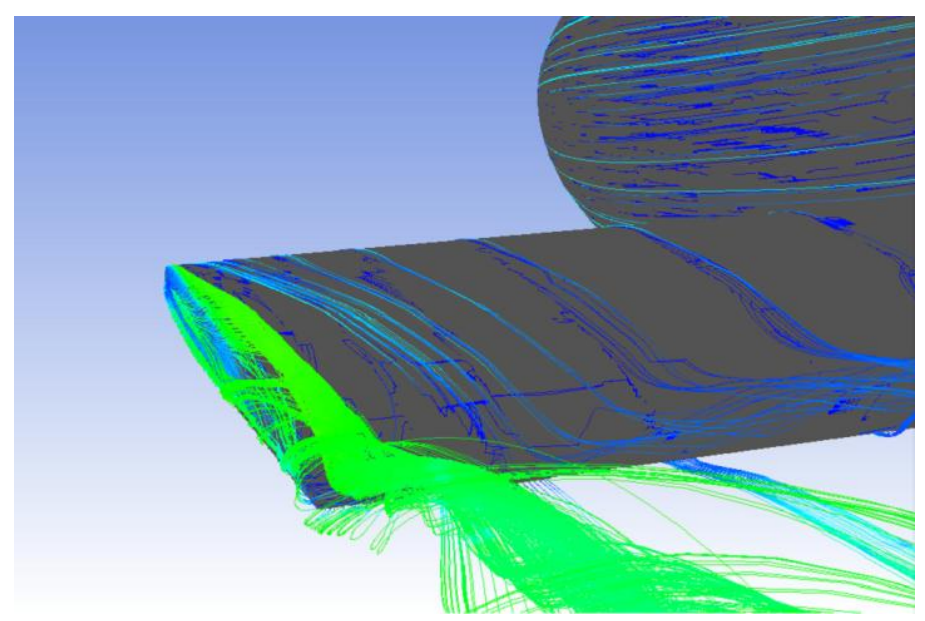

Figure 7-9 Streamlines of 'tip vortex' and inboard flow at wing tip of an SF25158_20. $0^{\circ}$ body angle. 9.5 incidence angle. Rectangular planform of $A R 8$. $\left(\operatorname{Re}_{\mathrm{c}}=120,000\right)$. 
Vortex generators were first used for the purpose of boundary layer control by Bruynes and Taylor in 1947 [35][36]. Boundary layer control consists of altering the flow characteristics of the boundary layer by the injection or removal of fluid into or from the boundary layer [37]. A vortex generator achieves this by transporting energy into the boundary layer through helical mixing of the high momentum fluid particles of the 'external' stream. This effectively sweeps away the retarded air, increasing the momentum of the fluid particles near the surface, promoting pressure recovery and preventing separation [38][39].

Vortex generators can take many forms: vane-type generators (introduced by Bruynes and Taylor) which are essentially small plates set at an angle relative to the flow producing a single vortex; other examples include ramps, wing-type, etc. These are usually small pieces of sheet metal or other material attached to the wing surface. However, some vortex generators are comprised of the wing itself, using the available upper/lower-surface pressure gradient. Examples of these types are the leading-edge discontinuity and the leading-edge notch and are illustrated in Figure 7-10.

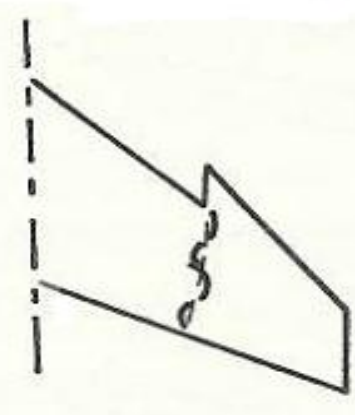

(a) Leading-edge discontinuity or "snag".

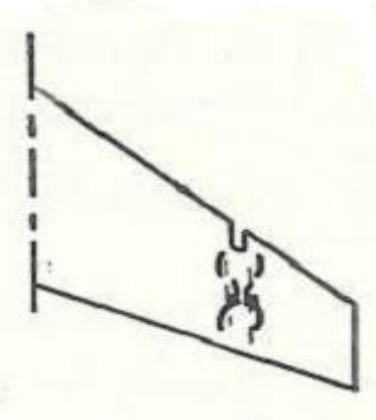

(b) Leading-edge notch.

Figure 7-10 Planform-based vortex generators from Pearcey [39]. 


\subsection{Downwash Conjecture}

To understand why a discussion of the tip vortex system is of interest in regards to the performance of ShopFoil, refer the spanwise plots of the velocity magnitude contours near the tip of the SF25158_20 in Figure 7-11 below. The region highlighted by the red box indicates inhibited boundarylayer growth and no separation through the trailing edge, represented here as a low velocity region (denoted by blue), which is inconsistent with the rest of the span. It can be seen to the right of this highlighted region that as the flow progresses toward the trailing edge the boundary-layer grows and separates.
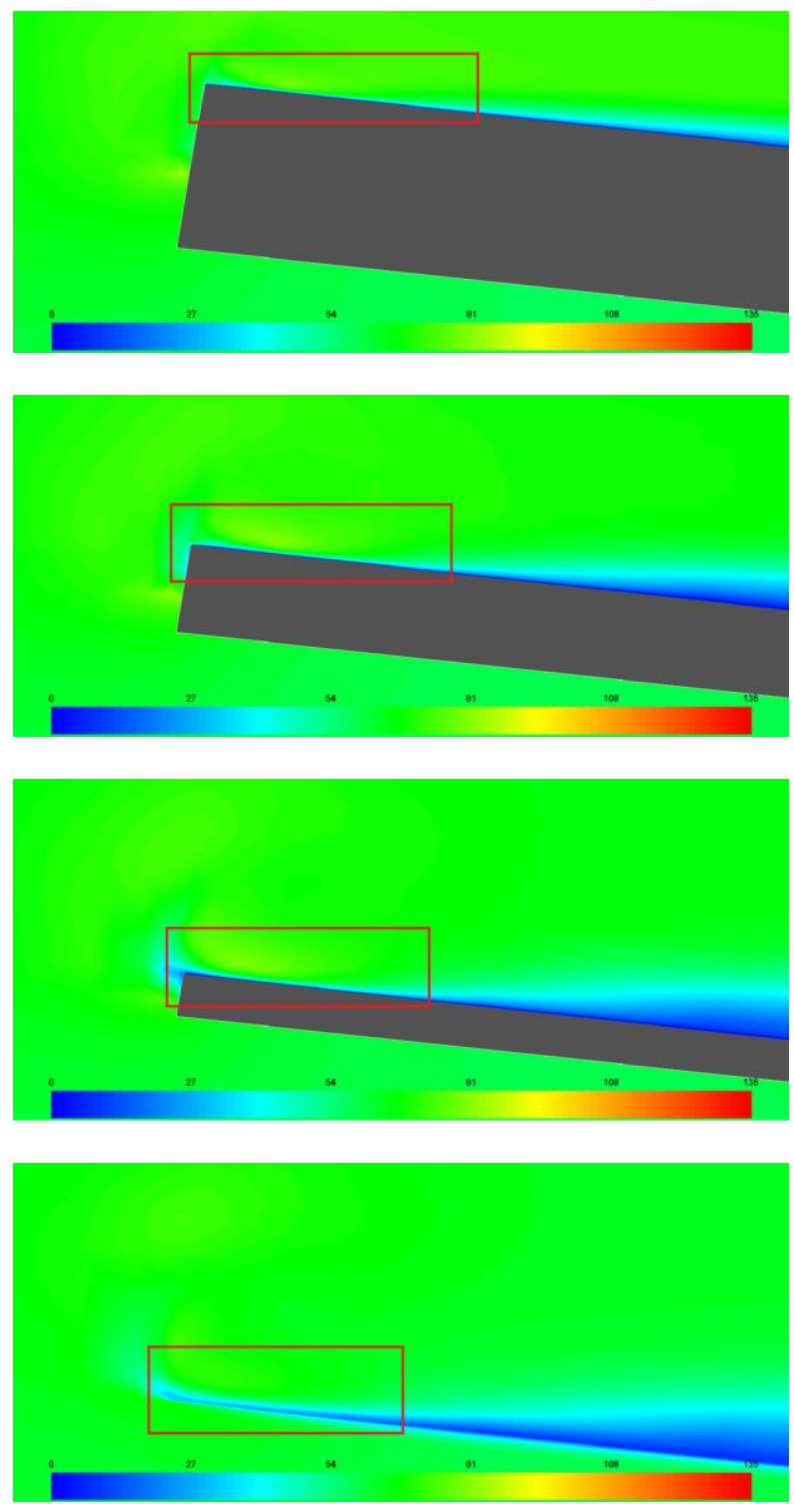

Figure 7-11 Contours of velocity magnitudes at successive chordwise locations near tip of SF25158_20 at $\alpha=$ 11.6 ${ }^{\circ}$. The images display spanwise slices from the mid-chord to the trailing edge. 
The question arises whether this occurrence is simply the result of downwash reducing the effective angle of attack to a region which naturally maintains attachment, or whether this is the result of three-dimensional effects such as tip vortex interaction. In order to proceed with the discussion on the potential influence of the 'tip vortex' on the performance of the ShopFoils, it is necessary to demonstrate to the extent the available evidence allows that downwash and its resulting 'induced angle of attack' cannot be the sole cause of the delayed separation at the wing-tips and thus the stall progression pattern of a rectangular wing. In order to justify this it is necessary to establish some means to differentiate between the flow contribution of downwash and that of the tip vortex. An assumption in the form of a conjecture will be made to establish a grounds upon which this can be achieved.

\section{Downwash Conjecture}

According to Prandtl's Hypothesis, the lifting-line theory assumes each section of the wing acts as though it is an isolated two-dimensional section at angle of attack $\alpha_{0}$ [40]. As a direct result of Prandtl's theory, downwash acts to influence the effective angle of attack of these isolated sections and does not account for any three-dimensional flow effects along a wing surface. For a finite rectangular planform, the vectorial contribution of downwash to the flow over a wing is limited to the components comprising a two-dimensional plane coincident with the wing cross-section. Ideally, the flow characteristics over a standard finite wing may be conceptually simplified to a consist of a superposition of two-dimensional contributions, three-dimensional pressure gradient effects, tip-vortex impingement on the wing surface, shed vorticity influence through downwash, and potential turbulent effects. In other words, the two dimensional data serves as a basis for the contribution that downwash has on the flow over the same cross-section on a three-dimensional wing. Any deviation from this basis is the result of one or more of the three-dimensional influences.

Therefore, it can be assumed that the flow characteristics observed for a given two-dimensional cross-section at some spanwise location of a three-dimensional wing at some $\alpha_{\text {eff, }}$ which cannot be shown to exist for the same section and equivalent geometric angle of attack in a pure two-dimensional case cannot be solely an effect of downwash but must, in part, be the result of some three-dimensional effect, (e.g., spanwise flow, turbulence, tip vortex impingement, etc.).

This conjecture establishes the grounds by which to make this distinction through a comparison of per section (quasi two-dimensional) data from a finite wing with the actual two-dimensional results. Ideally, a quantitative comparison would be made; requiring the determination on a per section basis of $\Gamma(y), w(y)$ and the resulting $\alpha_{e f f}(y), C_{l}$, and $C_{d}$; then directly comparing with the two-dimensional lift and drag data at $\alpha_{e f f}(y)$. However, these comparisons will be qualitative and based on velocity contour plots via the downwash conjecture. 
Velocity contour plots were made for representative cross-sections of the region highlighted in red from Figure 7-11 and qualitatively compared to the two-dimensional velocity contours. Determining the effective angle of attack at each wing section for a direct comparison to the equivalent twodimensional angle of attack is not a trivial task and thus required an alternate approach; due to the difficulty in comparing against every angle of attack the process was simplified with some assumptions. First, it was assumed that at every section across the span the lift is greater than or equal to zero. This assumption eliminated the need to consider angles of attack with negative lift coefficient. Additionally, there was no need to consider all positive lift angles of attack as the least amount of separation takes place at the smallest lift coefficient; for the SF25158_20 this occurs at approximately $-5^{\circ}$, however, this comparison utilized data from $-4^{\circ}$ and $-6^{\circ}$. If it could be shown that the growth of the boundary layer at the lowest positive lift coefficient is larger than that of the section of interest on the wing then there is a three-dimensional effect preventing its growth and delaying separation, rather than an effect of downwash only. The velocity contour plots in Figure 7-12 were made for various sections within the region of interest and are in agreement with the spanwise plots of Figure 7-11 which indicate little to no boundary layer growth (indicated by the arrows) over the trailing-edge.
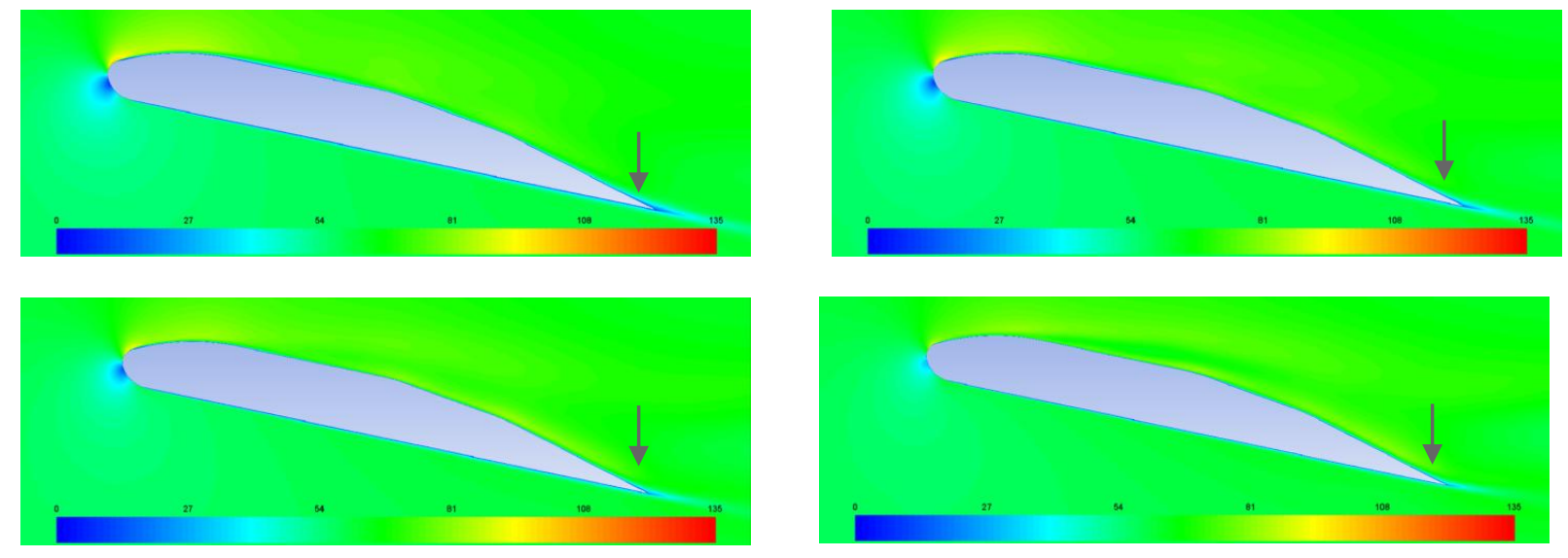

Figure 7-12 Velocity magnitude contours for cross-sections within the highlighted zone at the wing tips from Figure 7-11. Velocity scale is from $0 \mathrm{~m} / \mathrm{s}$ (blue) to the free stream velocity of $60 \mathrm{~m} / \mathrm{s}$ (green) to $135 \mathrm{~m} / \mathrm{s}$ (red).

In comparing these sections with those in Figure 7-13 it is apparent that the boundary layer profiles for the $-4^{\circ},-6^{\circ}$, and $0^{\circ}$ angles of attack do not show the same profile. The $-6^{\circ}$ case maintains the smallest boundary layer thickness but there is still a consistent growth beginning at the angle just before the trailing edge. Similarly, the $-4^{\circ}$ shows a thickening layer but with a greater rate of growth. These two data points sit at either side of the zero lift angle of attack and thus show that we should not find a match for the contours in the vicinity of the tip at $-5^{\circ}$. Further, every angle of attack beyond $-4^{\circ}$ produces increasing boundary layer thickness and eventually separation indicated by the contours at $0^{\circ}$. 


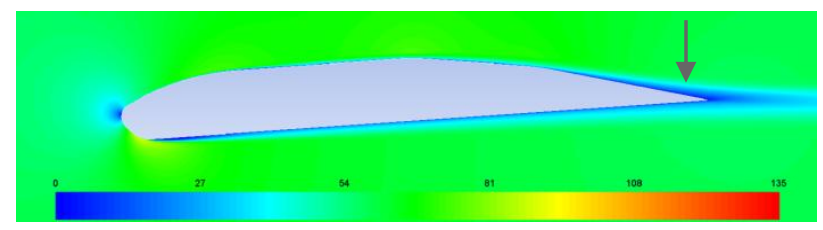

a) SF25158_20 at $-4^{\circ}$.

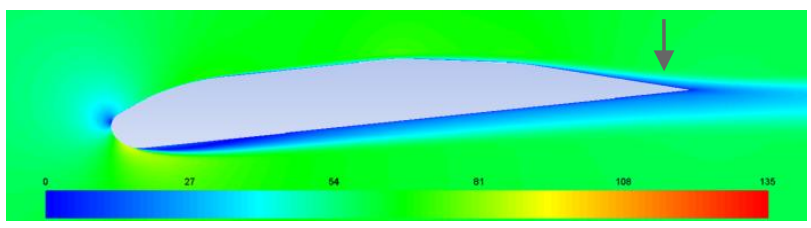

b) SF25158_20 at $-6^{\circ}$.

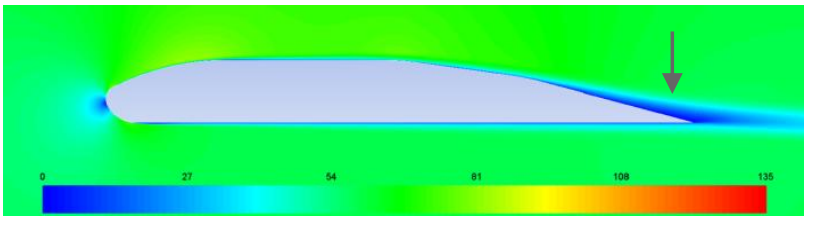

c) SF25158_20 at $0^{\circ}$.

Figure 7-13 Two-dimensional velocity contour sections at $-4^{\circ},-6^{\circ}$ and $0^{\circ}\left(\operatorname{Re}_{c}=120,000\right)$. (Velocity in $\left.\mathrm{m} / \mathrm{s}\right)$

It can be concluded from this qualitative analysis that there is no match for the trailing-edge profile characteristics of Figure 7-12 in the two-dimensional contours at any relevant angle of attack. Therefore, the possibility that downwash is the culprit behind the phenomenon at the tip has been ruled out and there must be some three-dimensional effect which is inhibiting boundary layer growth in this region and preventing separation.

\subsection{Comparative Advantage}

It has been concluded that some three-dimensional effect has a role in diminishing the growth of the boundary layer near the wing tip and thus preventing separation. The region in the vicinity of the tip was further investigated by producing spanwise plots of the $\mathrm{x}$ and $\mathrm{z}$ velocity components at four chord locations, which are provided in Figure 7-14. From these plots it appears that the spanwise flow typically associated with the upper surface of the wing due to the pressure gradient at the tip, that which moves inboard, plays no role in the prevention of boundary layer growth at the trailing edge; there is no indication of an inboard component near the tips. The data indicates a relatively large spanwise zcomponent of velocity in the outboard direction (i.e., positive values) is directly contacting the wing surface. 

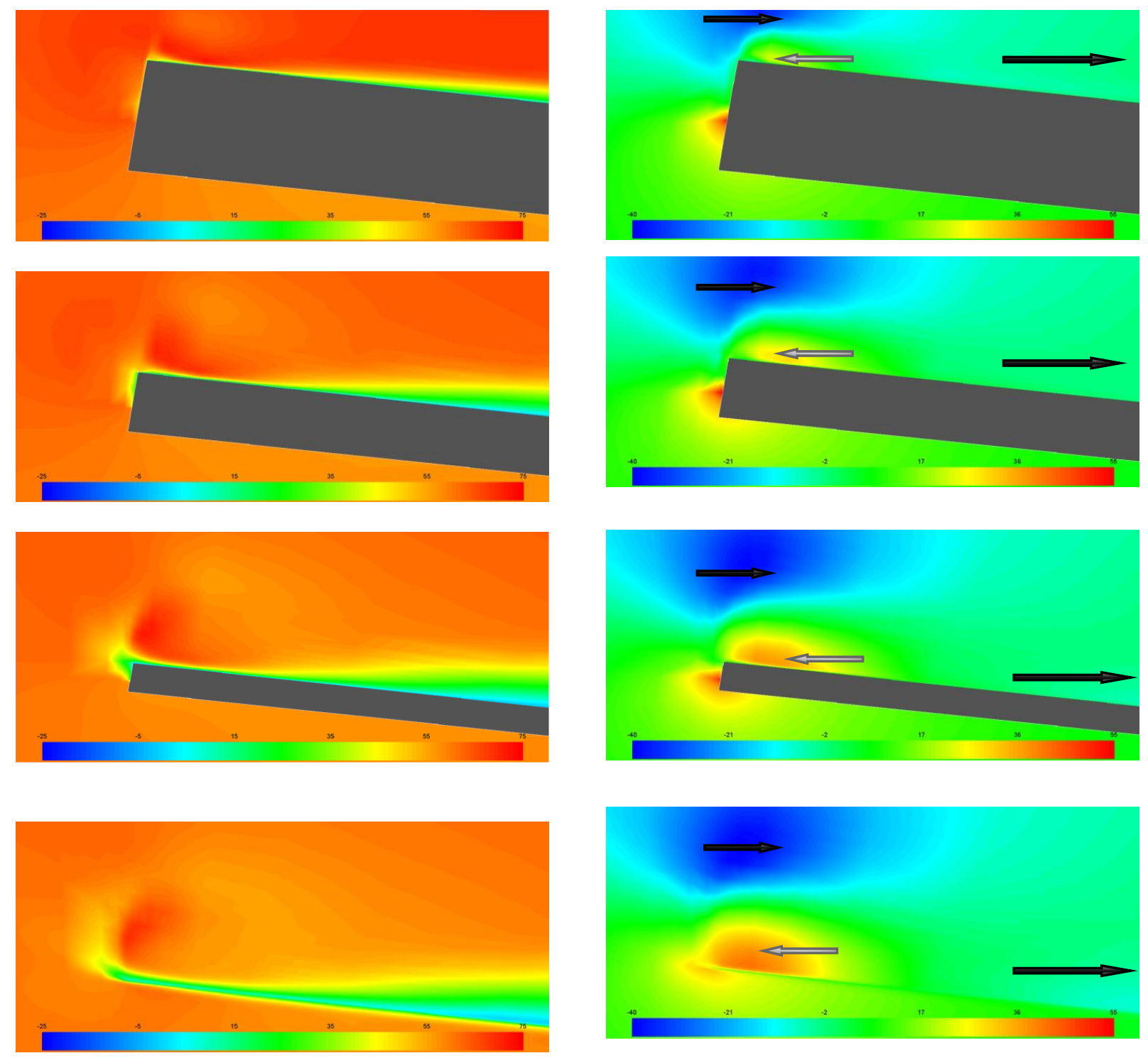

Figure 7-14 Velocity component contours at tip of $\operatorname{SF25158\_ 20}$ at $11.6^{\circ}\left(\operatorname{Re}_{c}=120,000\right)$. Streamwise $x-$ component (Left); Spanwise z-component (Right). (Velocity in $\mathrm{m} / \mathrm{s}$ )

This is also confirmed by the cross-section contours of z-velocity near the tip as in Figure 7-15, showing the spanwise component impinging on the surface (colored grey) of the trailing edge (blue region is moving into the page and red is moving out). 


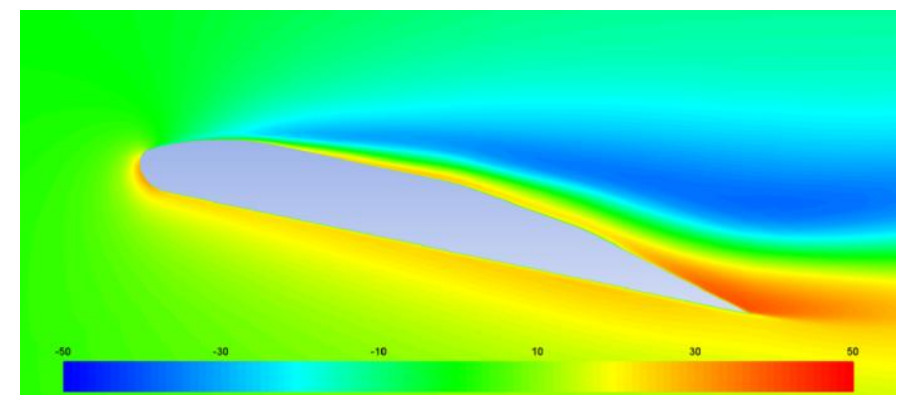

Figure 7-15 Wing-tip cross-section contours of z-velocity at $11.6^{\circ}$ showing interaction between the 'tip vortex' and the wing surface $\left(\operatorname{Re}_{\mathrm{c}}=\mathbf{1 2 0 , 0 0 0}\right)$. Blue contours are pointing into the page, red are coming out of the page. (Velocity in $\mathbf{m} / \mathbf{s}$ ).

Upon consideration of the location of the tip vortex and the plots of its velocity components, it seems likely that the vortex system is playing a role in the momentum exchange of the retarded flow in the boundary layer along the trailing edge; in effect acting as a vortex generator. If this is the case then it is possible that for some segment of the span near the tip that the vortex which would otherwise be a source of loss is inducing its own "energy recovery" to some extent by reducing the pressure drag associated with separation. It may be noted, however, that the tip vortex is present for all rectangular wings and thus this cannot benefit. To explain why this is possible the concept of "comparative advantage" is introduced.

Refer to the profile drag coefficient plot of Figure 7-16 (a). For some angle of attack, say $8^{\circ}$, numerical results indicate that the ShopFoil is producing approximately 70\% more drag than the SD6060. By separating the viscous and pressure drag components from the total profile drag as shown in Figure 7-16 (b) it can be seen that the viscous component of drag is generally equivalent for both airfoils, while the major difference is the pressure drag component, that which is mostly owing to separation. The ShopFoil has almost twice the pressure drag of the SD6060. In the above scenario, where for some span near the wing tips, separation is prevented, the planform comprised of an airfoil of higher relative $\mathrm{C}_{1}$ and higher relative pressure drag will gain an advantage in L/D because it has more to gain from the elimination of this drag component relative to its counterpart. Thus, over the extent of that portion of span (in this case approximately $10-15 \%$ of the half-span), an airfoil such as the ShopFoil may outperform the SD6060 relative to the two-dimensional data because it gains a comparative advantage under the influence of the tip vortex. 


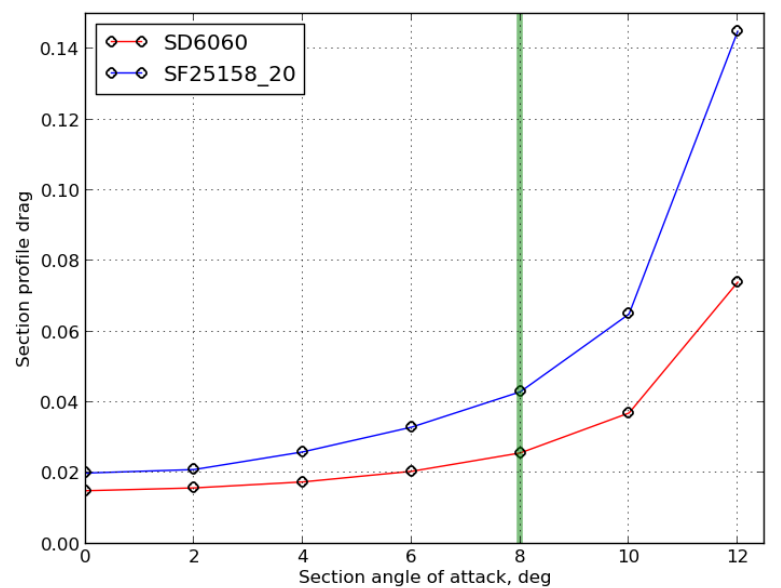

(a) Profile drag comparison.

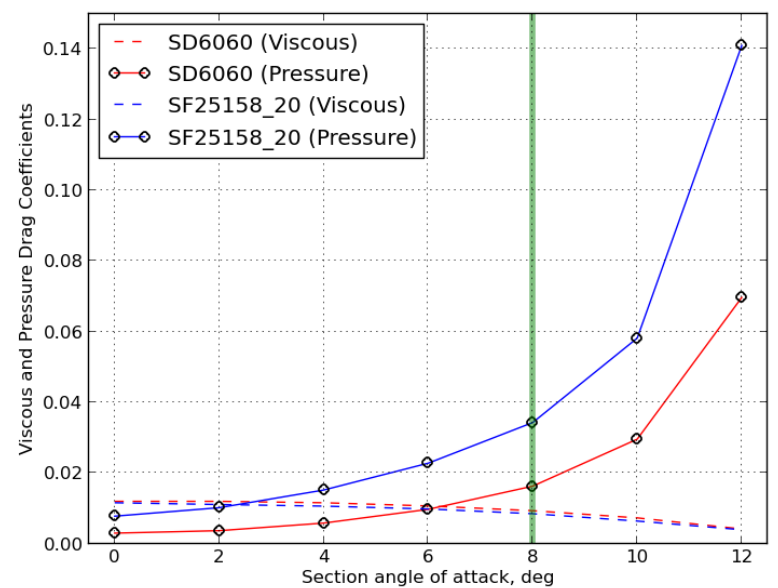

(b) Viscous and pressure drag components.

Figure 7-16 Profile, viscous, and pressure drag comparisons $\left(\operatorname{Re}_{c}=120,000\right)$. 


\section{Chapter 8 Conclusion}

\subsection{ShopFoil and HP Requirements}

Determining whether ShopFoil could meet the requirements of the HP platform was the fundamental goal of this research. To achieve this, numerical methods such as XFOIL and CFD were employed. Their lift results were consistent, indicating the superior performance of ShopFoil over the SD6060 at angles of attack below $11^{\circ}$. While lift to drag results for the two-dimensional case indicated that the ShopFoil maximum performance was less than the SD6060 and peaked at lower angles of attack, this difference vanished in the transition to three-dimensional simulations for the selected ShopFoils. These results were comparable to alternate wind tunnel tests for the same platform not directly related to the research addressed in this work.

Based upon the data collected from numerical simulations and with supporting evidence from experimental results it can be confidently stated that certain ShopFoil sections are capable of meeting the glide ratio requirements of 4.5:1 for the HP platform.

\subsection{ShopFoil Performance}

The three-dimensional CFD simulation results indicated that the performance of certain ShopFoils was nearly indistinguishable from that of the SD6060, while experimental results seemed to support these findings even confirming that one of the sections was capable of out-performing it. This outcome was surprising as it was in disagreement with the two-dimensional results predicting reasonable lift-to-drag, but at lower angle of attack. Additionally, these airfoils are crude approximations to the standard sections, lacking the contours associated with aerodynamic efficiency. That they may be able to achieve the same results as a cross-section specifically designed for these low Reynolds numbers is puzzling.

Reasoning that this effect may be due to some mechanism that the numerical models were incapable of capturing (e.g., a trip effect caused by the sudden changes in surface angles; or that similar to that of the dragonfly wing an effective shape is generated), an investigation proceeded in the wind tunnel through two-dimensional flow visualization. 
Wind tunnel tests did not, however, reveal the presence of any such phenomenon or that the ShopFoils were able to delay detachment at angles of attack beyond that of the SD6060 through any means. While the ability of the ShopFoils to maintain attachment throughout the leading-edge and midchord is of interest, flow visualization provided no conclusive evidence as to how this was achieved and determination of such must be left to further research.

What testing did show was the difference between the nature of the separated flow in the real case and that predicted by the CFD turbulence model. These differences focused on separation progression. The turbulence model seemed to indicate separation at lower angles of attack. One possibility for this could be the difference in the nature of the shedding. It is difficult to make a definite determination but it is seems that the turbulence model is producing relatively large laminar eddies while the real flow is either generating turbulent shedding or smaller and higher-rate laminar eddies. However, these differences were consistent across the ShopFoil and SD6060 planforms and are likely not responsible for any relative differences between the two and three-dimensional results.

Having ruled-out any inherent and unique flow-control properties of the ShopFoil geometry and recalling that the shift in performance was associated with the transition to three-dimensional flow, a review of finite wing theory led to the consideration that downwash and the tip vortex system may be the source of this effect. As described in Chapter 7, the stream-wise shedding of the bound vortex along the span of a rectangular wing imparts a downward velocity resulting in a progressive reduction of the effective angle of attack from root to tip. The section of span in the vicinity of the tip is operating at a lower angle of attack where numerical evidence consistently shows the ShopFoils to dominate relative to the SD6060, particularly in regards to lift.

Additionally, for some segment of span near the tip which accounts for approximately $10 \%$ of the half-span, the influence of the tip vortex acts to prevent separation through momentum exchange of the external flow with that of the stagnated air in the boundary layer. Because the ShopFoils are prone to earlier separation and thus larger pressure drag than the SD6060 they gain a relative performance advantage or "comparative advantage" by exploiting the otherwise negative effects of the tip vortex.

In light of the current evidence, however counter-intuitive it may seem, these phenomena generally associated with adverse effects on wing performance are believed to act in cooperation to offset the differences between these airfoils; in effect, playing to the strengths of that which would otherwise be the lesser of the two. 


\subsection{Recommendations}

The initial goal presented in this thesis focused on developing an airfoil cross-section which could achieve a particular glide ratio while reducing the complexity of the fabrication process. The result of this was manifested through a particular rule set for the ShopFoil, which was defined in Chapter 2.4. However, this is just one of many possible alternative geometric definitions which can be explored. It may be useful to consider designs with differing leading-edge radius, symmetric sections rather than a single flat-bottom surface, increased number of surfaces and angles, inverted or concave surfaces, etc. The ShopFoil was inherently an experimental design, and this should continue in an effort to understand the potential for faceted design.

The second aspect of this thesis sought to resolve the physical flow mechanisms, if any, which were responsible for the comparable performance of the ShopFoil with respect to the SD6060 through flow visualization experiments. In pursuance of this research, it would be advisable to collect twodimensional lift and drag data to act in comparison to three-dimensional data. A similar method as was used in this paper to numerically isolate inherent two-dimensional characteristics from three-dimensional effects could be employed. This would help to determine whether these effects were resulting directly from the ShopFoil geometry. Additionally, flow visualization using smoke or particle image velocimetry (PIV) would possibly provide more detailed information regarding the presence of leading-edge bubbles and the nature of trailing-edge turbulence or separation. This could substantiate or rule-out their potential role in generating an effective airfoil shape. Further, more detail could be provided regarding the quality of the flow at the trailing edge. 


\section{References}

[1]. Manole, Leon R., Logsdon, Ernest L., Palathingal, Mohan J., Sebasto, Anthony J. Gun launched hybrid projectile. United States of America: Patent 8115149. 14 February 2012.

[2]. Hamburg, Shanti D. "Conceptual and Preliminary Design of a Stowable Ruggedized Micro Air Vehicle." MS Thesis. West Virginia University, 2010. Print.

[3]. Browning, P., Cain, R., LaBarbara, K., Huebsch, W., Wilhelm, J. "An Experimental Investigation of the transient Effects Associated with Wing Deployment During Ballistic Flight." SAE Int. Journal of Aerospace (2011): 4 (2). Print.

[4]. Loftin, Laurence K. "Quest for Performance: The Evolution of Modern Aircraft." NASA Special Publication SP-468 104-106. 1985. Report.

[5]. Selig, Michael S., Guglielmo, James J., Broeren, Andy P., Giguere, Philippe. Summary of LowSpeed Airfoil Data. Virginia Beach: SoarTech Publications, 1995. Print.

[6]. Selig, Michael S., John F. Donovan and David B. Fraser. Airfoils At Low Speeds. Virginia Beach: SoarTech 8, H. A. Stokely, 1989. Print.

[7]. Anderson, John David Jr. A History of Aerodynamics: And Its Impact on Flying Machines. New York: Cambridge University Press, 1997. Print.

[8]. Mises, Richard Von. Theory of Flight. New York: Dover Publications, 1945. Print.

[9]. Abbott, Ira H. and Albert E. Von Doenhoff. Theory of Wing Sections. New York: Dover Publications, Inc., 1959. Print.

[10]. Munk, M. M. "Elements of the Wing Section theory and of the Wing Theory." NACA Report No. 191. 1924. Document.

[11]. Jacobs, E. N. "Preliminary Report on Laminar Flow Airfoils and New Methods Adopted for Airfoil and Boundary-Layer Investigations." NACA WRL 345. 1939. Report.

[12]. Theodorsen, T. "Theory of Wing Sections of Arbitrary Shape." NACA Report No. 411. 1932. Document.

[13]. Eppler, R. "Direct Calculation of Airfoils From Pressure Distribution." NASA TT F-15, 417. 1974. Document.

[14]. Mueller, Bruno. boomerang-sport.de. 1999. Web Page. 20 March 2013.

[15]. Airfoils: Colorado Boomerangs <www.coloradoboomerangs.com/airfoils.html〉. n.d. Wed Page. 20 March 2013.

[16]. Kline, Richard L. and Floyd F. Fogleman. Airfoil for Aircraft. United States of America: Patent 3706430. 19 December 1972. Document.

[17]. Kline, Richard L. and Floyd F. Fogleman. Airfoil for Aircraft Having Improved Lift Generating Device. United States of America: Patent 4046338. 6 September 1977. Document.

[18]. Lumsdaine, Edward, Johnson, William S., Fletcher, Lynn M., Peach, Judith E. "Investigation of the Kline-Fogleman Airfoil Section for Rotor Blade Applications." NASA-CR-141282. 1974. Report.

[19]. Liberatore, Anthony J. "Looking at the Facetmobile From a New Angle." EAA Experimenter 99484. EAA, January 2010.

[20]. Wainfan, Barnaby and Hans Nieubert. "Feasibility Study of the Low Aspect Ratio All-Lifting Configuration as a Low-Cost Personal Aircraft." NASA LARC NAG-1-03054. 2004. Report. 
[21]. Tamai, Masatoshi, Wang, Zhijian, Rajagopalan, Ganesh, Hu, Hui. "Aerodynamic Performance of a Corrugated Dragonfly Airfoil Compared with Smooth Airfoils at Low Reynolds Numbers." 45th AIAA Aerospace Sciences Meeting and Exhibit (AIAA-2007-0483). Reno, Nevada: AIAA, 2007. Conference Paper.

[22]. Kesel, A. B. "Aerodynamic Characteristics of Dragonfly Wing Sections Compared With Technical Airfoils." The Journal of Experimental Biology, 203 (2000): 3125-3135. Print.

[23]. "Flow Visualization of a Dragonfly Wing." Micro Flying Robot Laboratory, Nippon Bunri University. Image.

[24]. Lissaman, P. B. S. "Low-Reynolds-Number Airfoils." Annual Review of Fluid Mechanics: AeroVironment Inc., 15 (1983):223-239.

[25]. McMasters, J. H. and M. L. Henderson. "Low-Speed Single-Element Airfoil Synthesis." NASA/SSA Third International Symposium on the Science and Technology of Low-Speed and Motorless Right, NASA Langley Research Center. NASA CP, 1979. 2085 Pt. 1.

[26]. Carmichael, B. H. "Low Reynolds Number Airfoil Survey." Volume I, NASA Contractor Report 165803. 1981.

[27]. Roberts, W. B. "Calculation of Laminar Separation Bubbles and Their Effect on Airfoil Performance." AIAA Journal 18.1 (1980): 25-31. Print.

[28]. Drela, Mark. XFOIL: An Analysis and Design System for Low Reynolds Number Airfoils. Cambridge: MIT Dept. of Aeronautics and Astronautics. Report.

[29]. Menter, F. R. "Two-Equation eddy-Viscosity turbulence Models for Engineering Applications." AIAA Journal, vol. 32, no 8. (1994): 1598-1605. Journal Article.

[30]. Napolillo, Zachary G., et al. "ShopFoil: Design of Unoptimized Airfoils." ASME 2012 International Mechanical Engineering Congress \& Exposition. Ed. ASME. Houston: ASME, 2012.

[31]. Bertin, John J. Aerodynamics for Engineers. Upper Saddle River: Prentice Hall, 1979. Print.

[32]. Prandtl, L. Applications of Modern Hydrodynamics to Aeronautics. Gottingen University Report No. 116. Gottingen: Gottingen University.

[33]. Hoerner, Sighard F. Fluid Dynamic Lift. Mrs. L. A. Hoerner, 1965. Book.

[34]. Hoerner, Sighard. Fluid Dynamic Drag. Mrs. L. A. Hoerner, 1965. Book.

[35]. Bruynes, H. Fluid Mixing Device. United States: Patent 2,558,816. 3 July 1951. Patent.

[36]. Taylor, H. D. "Summary Report on Vortex Generators." R-05280-9. 1950. Report.

[37]. Flatt, Joseph. "The History of Boundary Layer Control Reserach in the United States of America." Lachmann, Gustav Victor. Boundary Layer and Flow Control, Its Principles and Application. New York: Pergamon Press, 1961. 122. Print.

[38]. Cooke, J. C. and G. G. Brebner. "Separation and its Prevention by Geometric Design." Lachmann, Gustav Victor. Boundary Layer and Flow Control, Its Principles and Application. New York: Pergamon Press, 1961. 173-174. Print.

[39]. Pearcey, H. H. "Shock-Induced Separation and its Prevention." Lachmann, Gustav Victor. Boundary Layer and Flow Control, Its Principles and Application. New York: Pergamon Press, 1961. 1277. Print.

[40]. Pope, Alan. Basic Wing and Airfoil Theory. New York: McGraw-Hill, 1951. Print. 